Johnston, John Robert

Flora of the islands of Margarita and Coche, Venezuela 


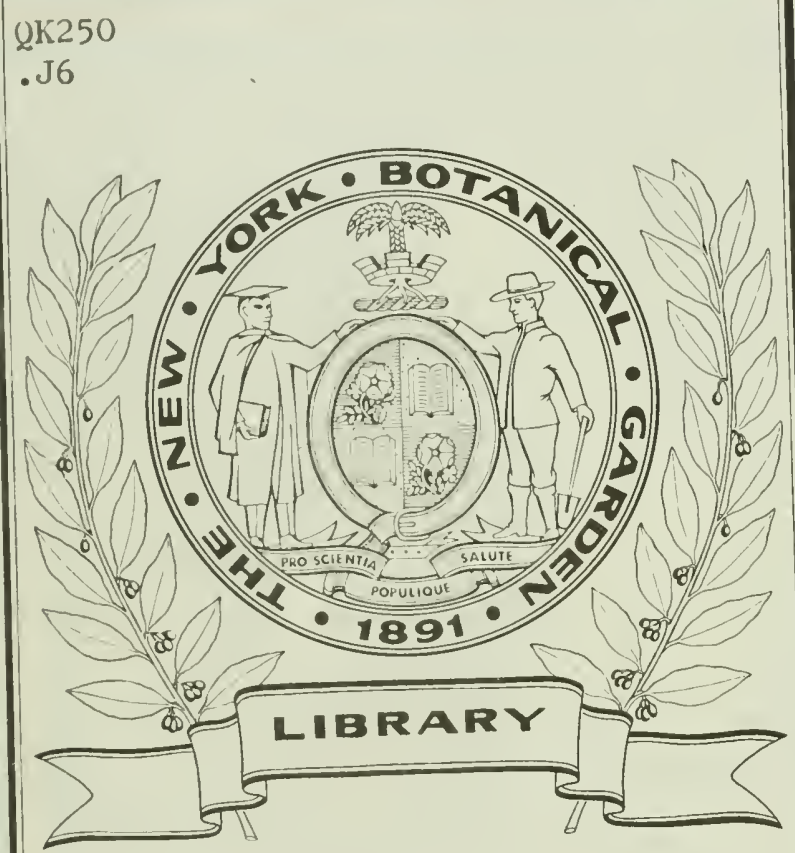






\section{Proceedings of the Boston Society of Natural History}

VoL. 34, No. 7 ,

p. 163-312, pl. 23-30.

CONTRIBUTIONS FROM THE GRAY HERBARIUM OF HARVARD UNIVERSITY. NEW SERIES.-NO. XXXVII. FLORA OF THE ISLANDS OF MARGARITA AND COCHE, VENEZUELA.

BY JoHN ROBERT JOHNSTON, M.S.

BOSTON:

PRINTED FOR THE SOCIETY.

JUNE, 1909. 

No. 7.- CON'TRIBU'TIONS FROM THE GRAY HERBARIUM OF HARVARD UNIVERSITY. NEIV SERIES.- NO. XXXVII. FLORA OF THE ISLANDS OF MARGARITA AND COCHE, VENEZUELA.

BY JOHN ROBERT JOHNSTON, M. S.

\section{Contents.}

General considerations on the flora of Venezuela - Variety of vegePage. tative conditions - Desirability of further collecting - Exploration and botanical work . . . . . . . . 163

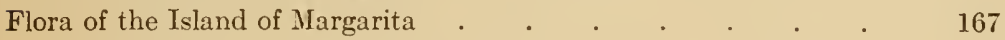
Introduction . . . . . . . . . . 167

Physical features - Topography - Rainfall and clouds Temperature . . . . . . . . . 168

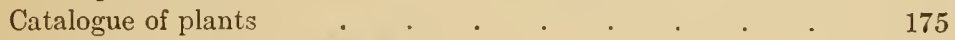

The economic plants of Margarita $\quad$. $\quad$. $\quad$. $\quad$. $\quad$. 270

Distribution of the plants - Regional distribution - Grouping - Paucity of specimens - Adaptation and barriers to dispersal - Seasonal distribution

Composition of the flora - The cultivated, the cosmopolitan, and the plants of restricted distribution - The variety of plants and the groups most largely represented . . .

The flora of the Island of Coche

Description of the vegetative conditions . . . . .

Catalogue of plants

Comparison of the flora of Margarita and Coche with that of other regions - Methods of comparison - Other Venezuelan islands - The mainland - The West Indies - Southern Conclusion United States Jiterature of geographical and botanical publications . . . 304

\section{General Considerations on the Flora of Venezuela.}

Professor Goebel's ('91) most interesting account of the vegetative conditions to be found on the Cordilleras of Merida in Venezuela is descriptive of an excellent field for botanical research. Within 150 kilometers (93 miles) of the shores of Lake Maracaibo the moun- 
tains rise to an altitude of 4000 meters $(12,000 \mathrm{ft}$.) so that a traveler in passing from the Lake up to the mountains traverses the lowland forests, the higher and barren hills, the rich valleys, the forested mountain sides and gorges, to the treeless summits, some of which are capped with snow.

In this short range occur all variations from the swampy seashore and the barren hills to the luxuriant valleys, and the alpine regions. On the mountain sides oceur furthermore at various altitudes not only quiet gorges in which the woods are dripping with moisture but also knolls exposed to the fierce winds, often accompanied by great changes in temperature. A greater variety of vegetative conditions is difficult to find within so small an area in any other country.

Venezuela may well be considered as consisting of three different regions: the Andean extending from Lake Maracaibo southwest over the mountain ranges to Colombia; the coastal region from Lake Maracaibo eastward to the Gulf of Paria at Trinidad, including all the coastal islands and extending inland to the llanos by the Orinoco; and the third region consisting of the Orinoco district and all of the extensive and little known forests to the south. While each of these three divisions shows some of the characteristics of the others, each has features of interest peculiar to itself, the first region being alpine, the second consisting of the barren or desert lills of the coast and of the islands, and the third being characterized by the grassy plains and forest areas of the llanos and selvas.

Such a variety of conditions as is presented in this country leads one to expect much of botanical interest. There must be both a remarkable variety of plants and many interesting adaptations of the plants to their environments. A number of collections lave been made, but not nearly so many as the conditions warrant, and unfortunately the reports of these collections are seattered and incomplete. The need for more work on the flora of Venezuela is strongly felt. Brazil has the elahorate work of Martius ('40-'06); the Guianas those of Schomburgk ('47-'48) and Pulle ('06); Colombia that of Kinsten ('58-'69) and also the recent collections of Sinith and Pittier. 'The Viest Indies as a whole have Grisebach's ('64) well known Flora, and the recent Symbolae Antillanae of Professor Urban ('03). But in Venezuela, lying between these lands, no botanical work at all complete has been done.

The first recorded visit of a naturalist to Venezuela is that of Peter 
Loefling (1776). He sailed from Cadiz, February 15, 1754, for Cumaná where he spent six months. Then he journeyed to New Barcelona, the missions of Piritu, and the river Guyana, where he stayed three months. Thence he traveled to the missions on the Curoni and returned to Cumaná. In the description of his travels are noted thirty-four plants occurring about Cumaná.

In the years from 1807 to 1825 were published various works by Humboldt, Bonpland ('14-'29; '14; '08) and Kunth ('15-'25) describing the travels of the first two and then giving notes on their observations. Their list of plants, collected for the most part about Cumaná and along the Rio Negro and Cassiquiari Rivers, comprises some 1200 numbers. Most of these are now in the herbarium of the Museum of natural history at Paris.

Funck and Linden landed at La Guaira in 1S40. Linden went to Peru while Funck went toward the Orinoco, visiting Cumaná, Guana-Guana, San Augustin where he made a rich collection, the Grotto de Guacharos, Caripe and its mountains. In 1842 and 1845 he visited Santa Marta, San Sebastian, and Galipan, and thence went to Curaçoa and to the region of the Silla de Caracas. His plants are in the Delessert herbarium at Genera. There is no published list of them. Linden arrived at Caracis in January, 1St2, and in May of the same year sent plants to Delessert. 'This collection (235 species) contained plants from the Silla de Caracas and the Cerro de Avila. In May, 1St2, he left Caracas for the Andes of Merida. In January, 1St3, he sent from Merida to the consul of France at Maracaibo plants collected in the high Ancles of Truxillo and Merida. Linden's plants of Colombia, which may include his Venezuelan ones, are at the following places: University of Ghent, herbarium de Candolle (2066 species), herbarium of the University at Leipzig, and the herbarium of the Royal muscum at Vienna.

There are plants of Funck collected at Caracas at the herbarium of the Academy of sciences at St. Petersburg (S19 species).

Plants of Funck and Schlim (Reichenbach, '54b) collected in tropical America are in the herbariums of the universities of Ghent and Leipzig as well as in the Delessert herbarium.

Plants collected by Moritz (see Hampe '47a, '47b) are in the British museum, at the Royal botanical gardens in Berlin, in the herbarium of the Imperial botanical garden of St. Petersburg, and in that of the Imperial academy of St. Petersburg, of the Royal museum at Vienna, and of the University at Leipzig. 
Plants of Wagner collected in Panama and Ecuador are in the royal herbarium of Munich and University of Göttingen. Possibly these include his Venezuelan plants.

Karsten ('58-'69) who published the extensive flora of Colombia and the adjacent regions included serenty-nine plants collected in Venezuela.

Plants collected by Birschel at Caracas are in the Gray herbarium. Fendler's (see Eaton '61) collections of Venezuelan plants are in the herbariums of de Candolle, Delessert, Engelmann, Franqueville, University of Dublin, Gray herbarium, and British museum. August Fendler was a German botanist who lived in Colonia Tovar near Caracas from 1854-59. His collection comprised nearly 3000 numbers. There is no published list excepting that of the ferns and orchids, and a large part of the plants remain in the herbariums entirely or partially unidentified. Fendler was at one time an assistant at the Gray herbarium and his collecting was carried on to some extent under Dr. Gray's encouragement and patronage. It is believed that the set of his plants in the Gray herbarium is as nearly complete as any in existence.

Adolphus Ernst, who for a number of years was secretary of agriculture in Venezuela and also a professor at the University of Caracas, has contributed more to our knowledge of the Tenezuelan flora than any other man since Humboldt's time. Ernst had in preparation a flora of Venezuela but owing to his death in 1899 it was never completed. He did, howerer, publish numerous short articles pertaining to the regetation. A complete list of these occurs in the bibliography of his works published at Jena in 1900 (Ernst, '00b). The more important of these are the lists of the plants of Los Roques (Ernst, '72a), of La Tortuga (Ernst, '76b), of Margarita (Ernst, '86), and the list of ferns and of the orchids of Venezuela. The plants which he collected appear to be entirely inaccessible today. They are not to be found in the University museum at Caracas nor in the old National museum of natural history. It is possible that they have been sent to various European herbariums. As his lists contain merely the names of the plants with few or no notes, their identification in some cases must remain a matter of question.

In 1896, Professor H. H. Rusby ('96) and Roy W. Squires collected about the lower Orinoco. Their plants are in the New York college of pharmacy and in the Gray herbarium. 
In 1900, Captain Wirt Robinson and Dr. M. W. Lyon, Jr., collected at La Guaira, Macuto, and San Julian about sixty plants which are now in the U. S. national herbarium (see Johnston, '08).

In 1901, with a party of three others I spent the months of July and August on the island of Margarita. About 300 species of plants were collected. On another trip to the same island in 1903, we increased the known flora of Margarita to 654 species. At the same time a collection was made on the island of Coche and risits were made to Carupano, Cumaná, La Guaira, and Caracas to compare their floras. Again in $190 \overline{7}$, I was enabled to risit Tenezuela although no collecting was done on Margarita. Visits to Pampatar on Margarita and to Carupano, Cumaná, Barcelona, Guanta, La Guaira, Caracas, and Valencia on the mainland, all have been of value to me in comparing the flora of Margarita with that of adjacent regions.

It is beliered that the abore collections comprise all that have been made in Venezuela with the exception of a few by Venezuelans. In some cases the data are incomplete and unsatisfactory but they may nevertheless furnish a basis for further research work along this line.

\section{Flora of the IslaNd of Margarita.}

\section{Introduction.}

The island of Margarita is only a small part of Venezuela, nevertheless its flora has proved to be of considerable interest, particularly as revealing sereral new species and as increasing the known geographical distribution of other species. The island was visited in 1873 by Dr. Adolphus Ernst who published a report of the plants discorered. Captain Wirt Robinson visited the island in 1895 making a collection of the birds and mammals. In 1901 a party of four students of Harvard unirersity consisting of Austin $\mathrm{H}$. Clark, O. O. Miller, Walter P. Jenkins, and myself spent the months of July and August collecting specimens of animal and plant life. In 1903, through the kindness of one of the friends of the Gray herbarium of Harvard university, I was enabled to visit Margarita again. This time I was accompanied by Dr. Albert F. Blakeslee, who deroted himself to the collection of algae and fungi, and by Clifford Wilson, who assisted us in our work. These visits to Margarita, which are all that have been reported of scientific workers, taken together furnish fairly complete data as to the character of the flora and its vegetative conditions. 


\section{Physical Features.}

The island of Margarita as seen from the mainland thirty-two kilometers distant appears as two conieal mountains separated by a long stretch of lowland. It may be seen on the accompanying map that it consists of two irregular polygonal areas connected by a narrow strip of land, each of these areas rising from the lowlands of the plain to the foothills and mountain ridge of the center, thus giving the conical appearance in the distance.

Located about eleven degrees north, and sixty-four degrees west, Margarita is sixty-seven kilometers long and thirty-two wide. It is eleven kilometers from the desert islands Cubagua and Coche, and is the largest of the Venezuelan islands that extend along the coast from Curaçoa to Trinidad. The highest peaks of the two ends are about forty-two kilometers apart. The relative extent of plains, foothills, and mountains can best be understood by considering the two ends separately and in detail.

The mountains of the eastern end rise to an altitude of 795 meters and have many spurs jutting out in different directions. Surrounding the momntain and its spurs are the valleys and plains which in some cases extend to the sea. On the eastern and northem sides are what may be termed outlying mountains which are less extensive than the central mountain mass, San Juan Mountain, and border directly on the sea. The top of San Juan Mountain, between the altitudes 650 and 795 meters, is destitute of trees. Bare rock ledges jut out from beneath the low shrubbery. Occasionally the summit is in clear sunshine, but quite as often the heary clouds laden with moisture are rapidly flitting orer, now completely enveloping it, and now exposing it to the warmth of the sun. Within thirty meters of the top is one spring on the El Valle side, and fifteen meters below that is another. Though these are entircly exposed they never seem to dry up. The dense woods which cover the mountains above 400 meters collect and retain the moisture of the clouds, thus together with the two springs furnishing a source for small streams below.

On the map of the island it is shown that the mountain ridge as a whole not only extends from northeast to southwest, but also rises gradually from the west in a succession of hills to the highest point which is above El Valle and San Juan, thence lowering a little for a distance of one and one half kilometers, and then dropping abruptly 
down to the plain near Asuncion. Each of the successively higher hills of the restern end swerve to the south in the form of ridges sloping gradually to the open plain at San Antonio. From the highest point, however, there proceeds a very distinctive ridge between San Antonio and El Valle. This Soutl Hill, as named for the collector's conrenience, together with a similar hill to the north of El Valle, called North Hill, curres so as partially to enclose El Valle, the most characteristic valley of the island. Farther around the mountain to the northeast of it, is the large valley of Asuncion, and northwest of this is Tacarigua and at the mestern end again, on the north side, and opposite El Valle, is the valley of San Juan.

With this system of narrow vallevs surrounding the mountain, it may be expected that there are momntain torrents in the time of heary rains, and such is the case. The vallers of San Antonio and of San Juan, however, have only the dry river beds in ordinary weather, while the three other valleys alone have permanent streams.

The above-mentioned springs are the source of one river in $\mathrm{El}$ Valle which has been directed into a reservoir at the head of the valler at an altitude of fifty meters, whence it is piped to the villages of $\mathrm{El}$ Valle and Porlamar. There are ten public and a few private drinking fountains or "pelas" on the way, a distance of eight kilometers. The valley forks at its head so that to the northeast occurs a narrow ravine in which flows a small stream. This is used for laundry purposes, and is lost in the soil before it reaches the lower valley. There is also a short distance to the east of this another stream which is confined to private grounds on which are three concrete reservoirs provided for it.

The region between El Valle and Asuncion valley has no distinctive features, nor is there any stream. Although down in the valley of Asuncion proper there is only one stream bed and not much water, one and one half kilometers or more above the village at an altitude of 4.50 meters there are no less than eight stream beds of which six always contain running water. A trail passing through the forest at the above altitude from one side of the valley around the end to the other, furnishes an excellent opportunity to study these streams. The valley is most curved toward the southeast and gradually straightens out to the northwest. Beginning from the southeast the streams or stream beds were numbered in collecting merely for convenience, but the numbers may well serve here. 
'The first stream has a very shallow and rather indistinct bed. The land is wet and muddy on both sides of it. 'The second stream is a little larger and forms distinct pools at intervals. Below the trail this river has a very steep bed and appears on the surface only oceasionally from underneath the rocks. Intervening between this and the next river is a slight elevation which extends as a small ridge into the valley. On the northwest side of this ridge is a ravine about twenty meters deep, at the bottom of which runs the third river. The southeast slope is very steep, the northwest is a gradual rise. A little farther on is another ravine of similar depth and its southeast side is almost perpendicular. The northwest slope is gradual. A short way from this is the third and deepest ravine containing the fifth river. Another and shallower ravine is near by containing the sixth river. These four ravines are similar in having a steep slope on the southeast side and a gradual rise on the opposite side. The unimportant dry river beds of the seventh and eighth streams are but a short way farther, very near to the ridge separating the large Asuncion valley from that of Tacarigua.

The valley of Tacarigua, the next in the series around the mountain, is very long and has steep sides. The one river, the Rio Blanco, flows down to the bottom of the valley where it is lost eleven or twelve kilometers from the sea. The water is very impure though from what source I do not know. It is slightly muddy in color and tastes similar to a sulphur spring. Adjoining the valley of Tacarigua is that of San Juan, the last of the series surrounding the mountain. As this has nothing but a dry river bed it is of little importance.

As has been said, the mountain as a whole is covered by dense woods. The summit of San Juan Mountain is the only exposed part. The highest part of all the ridges is covered with shrubs which lower down gradually give place to small trees and still lower to the very tall trees. In all parts rocks are projecting in an irregular fashion. Not even in the densest part of the woods is there a soil of any considerable depth, and of course the river beds are extremely rocky. Where the mountain slope is interrupted by some hill, there the woods stop, but where the slope is continuous with a valley the woods extend to a much lower level. This latter condition occurs characteristically in El Valle and in the valley of Asuncion which are the only two worthy of detailed consideration.

The woods of El Valle are heavy as low as fifty meters, the height 
of the reservoir, although they are rapidly being cleared off in all directions. Immediately below this is the extensive coconut grove reaching down into Porlamar. The configuration of the land about the village of El Valle makes it an ideal place for botanical study. North and South Hills as before mentioned leave the mountain in a southerly direction and both give off short ridges running toward each other and also away from each other on the other side.

About a kilometer and a half from the mountain, South Hill turns to the southeast, and North Hill turns to the southwest, the two stopping but a short way from each other, thus leaving a narrow entrance into a nearly enclosed valley. In this small area there are exposures to all points of the compass. There is one dry river bed extending from the foot of the mountain to the sea, that is important in the rainy season. It is here that the heavy vegetation of the valley grows.

Asuncion is the only other valley of importance. The head of it has been described in the discussion of the mountain rivers. The lower part is quite open and is continuous with the plains to the sea. This valley faces to the northeast so that it gets the wind and moisture. The clouds, however, are usually at an altitude of from 400 to 600 meters so that it is only the upper part of the valley that is extremely moist, the richest part of the island. Otherwise the valley is similar to any of the lowlands.

Intervening between the valleys, partially surrounding them, and also standing as outlying features are the hills and small mountains conspicuous by their red soil and by their desolation. In the case of the hills attaining the height of 300 meters the summit has a few scattered trees. Other vegetation is either lacking or restricted to small patches of dry bushes. The soil is very conspicuously red, or on a few hills made gray by the preponderance of limestone. It is loose and easily weathered into small slabs or into a crumbling mass, which rapidly washes or rolls down the steep hills. At the time of the heavy showers the hills are gullied everywhere. As a consequence of their condition the soil is thin and can maintain only shallowrooted vegetation.

From the very nature of the rock it is to be expected that caverns of some size are to be found. Within twenty meters of the summit of Bat Cave Peak is a large cave which opens above near the summit, and also at the side. About one hundred meters from the foot of the hill there is a hole in the rocks through which I could with diffi- 
culty lower myself. Below was a cave about three meters deep and large enough to hold several men. Both of these cares are inhabited by bats, and the lower one contains numerous lizards.

'The barrenness of the hills is increased several fold in the plains excepting at the mouth of a valley. The surface of the plain is undulating only slightly and in few places. It is for the most part sandy, reckdish near the hills and white toward the sea. In places, notably between San Antonio and the sea, the surface is covered with irregular broken rock, small fragments, angular, and rery little weathered. By the sea the plains contain several lagoons as may be noted on the map.

On the plain a mile inland from Juan Griego is a small pond of brackish water always turbid and used merely for laundry purposes. At Punta Moreno is a small lagoon connected by a narrow outlet to the bay of Porlamar. At Punta Mosquito is Laguna Chica surrounded by mangroves. This is about six kilometers long and navigable by sloops. At Punta Mangles and at Punta Piedras are also small lagoons and mangrove swamps. The lagoon at Punta Piedras is open to the sea by an inlet about one meter deep and six meters wide. Inside the mangrove border is a clear circular body of water about one-half kilometer across.

From the above description of the eastern end, it is hoped that a good idea of the phrsical features may be dericed. When the distribution of the plants is discussed the picture of the district will be more complete. Then one may see along the shore at intervals the mangrove-bordered lagoons, then stretching inland the sandy plain dotted with the cactus and low shrubs exposed to the burning sun; the brown hills with small dead bushes, the valleys with the waving coconut palms and higher up the green-clad mountain sicle with the top reaching into the clouds.

The land intervening between the two ends is for the most part a large mangrove swamp, open to the sea on the north side and a short distance on the south side. Although the lagoon is about eleven kilometers long, it has very little clear water.

'The westem end of the island is of much less importance than the eastem. The conditions recorded here are taken from the meager encyclopedic references and from the observations which I made from the distant San Juan Monntain and on the Coche-Punta Piedras royage and on board the steamship from Porlamar to La Guaira and also from reports of the natives. 
According to encyclopedias, the mountain peak of this end is 60 meters higher than that of the eastern end. 'Though in the distance that looks very probable, it makes little difference in the character of the regetation. Little of the mountain range anywhere approaches the height of the peak. Most of the ridges are very steep and apparently have no forest growth, whatever. Cliffs are exposed in numberless places. Under such conditions, although the peaks are often hidden in the clouds there can be little moisture retained and consequently no springs nor rivers, a condition which agrees with the reports of the natives. "The single town of Macanao on the west coast is supplied with "posa" water (rainwater and seepage collected in clay pits) and with imported water. One large ridge with short spurs runs east and west along the south shore, and to the northeast is a series of high hills. The only plain is on the northwest side. The character of the western end as a whole then is similar to that of the hills and plains of the eastern end.

The above paragraphs give some idea of the topography of Margarita. It seems adrisable also to discuss so far as possible the temperature and moisture as they affect the conditions of regetation.

The above description distinctly suggests that Margarita is a very dry island. During the time from July 3 to August 22, in 1901, there were only two heary rains in El Talle each lasting much less than an hour, and I did not observe any light showers. 'This it must be remembered was during the rainy season on the mainland only twenty-seren kilometers distant. At this time conditions seemed to be quite normal. Orange trees and the coconut palms were bearing fairly well, and the valley bottoms were filled with thriving weeds.

Observations made from June 29 to September 4 , in 1903, were very different. At this time the coconut trees were dying, the oranges had turned color on the tree when less than half grown, and scarcely a weed could be found. The island and especially El Valle was in a bad drought. According to the natives there had been no rain for two or three and some said even five years. This latter time is shown to be incorrect by my own observations in 1901, but at any rate it is suggestive of very little rain for several years past. Records for 1903 are as follows:-

July 1t. Heary rain in Porlamar; very light at El Valle.

July 17. Light showers at El Valle.

August 14. Very heavy rains on the mountain and down through 
El Valle. Trails on the hillside were in many places washed away. Gullies were deepened considerably. Rained from about midnight till 10 A. M. of August 15.

August 16. Light showers.

August 21. Tery heavy rain in El Valle. The heavy sheets of rain seemed to ascend one valley to the mountain top and retreat to ascend the other valley. The rain was very light on the surrounding hills. During the storm and immediately after it a continuous roaring sound arose from the valley. On investigation this proved to be the rapid falling of the water down the steep gulches. Farther down the small streams united and flowed into the "dry river bed." The two trails rumning for a distance of a mile from the foot of the mountain into the plaza at El Yalle proved themselves to be veritable rivers. They were loosely pared with stones and were walled on each side so that they held in the water well. A half hour after this rain the trails were still submerged, and uniting with the river near the plaza they formerl a perfect torrent at least until 7.25 P. м.

A very different factor from the rain is the heary mist which almost constantly hangs above the mountain. About one third of the time the mountains above 600 meters are in the clouds. Very often from night until 6 or $7 \mathrm{~A}$. . . the hills over 300 meters are in the cloucls but they are never cloudy in the daytime except in case of storms. The clearing of the woods which everywhere seems to be inevitable, is going on here slowly. Where previously all the vegetation was dripping with moisture now for the most part conditions are much drier because trees have been cut down thus allowing the sunlight to enter.

In the preceding paragraphs it is seen that compared with other tropical districts, Margarita has almost no rainfall. The mainland in the summer season has a shower nearly every day. So has Trinidad off the northeast coast of Venezuela. While my records are mostly from the south side of the island, yet excursions made to Juan Griego and that vicinity indicate that it is but slightly better off.

Although the island is desert-like in character yet from the fact that it gets the northeast trades, a comfortable temperature may be expected. My records average as follows: at 6 A. м., 29 degrees C.; at 1 P. M., the hottest, 32.4 degrees C. These records are from El Valle. Porlamar always seemed hotter than this. The coolest is of course on the mountain top at San Juan Mountain. The average coolest is 25 degrees $\mathrm{C}$., the single coldest record was $24.5 \mathrm{C}$. This 
is the most delightful temperature condition imaginable, often cloudy and always breezy. El Valle is comfortable, but the villages on the plains are in the daytime very warm. At night it is comfortable everywhere.

Catalogue of the Plants of Margarita.

Dr. Ernst in his visit to Margarita, in 1S73, spent the month of May collecting at Juan Griego, Santa Ana, and Asuncion. His collection amounting to 242 different species are the first plants reported from that island but unfortunately they do not seem to be available for reference today. Their location is unknown to me; certainly they are not accessible in any place in Tenezuela.

The only other collections from Margarita and the only ones readily available for reference today are those made by our parties in 1901 and 1903. In 1901, we collected orer 300 different species spending most of the time from July 4 to August 22 in El Valle and the plains toward Porlamar, with only occasional trips to Juan Griego, Asuncion, and Punta Moreno. In 1903, from June $2 S$ to September 4, we found the region about El Valle in such a drought that for our collections we had to resort to the mountains for the most of our work. One trip was made to Juan Griego, Tacarigua, and Asuncion, one to Peilar and sereral to Punta Moreno and Punta Mosquito. We also made one excursion to the island of Coche and to Punta Piedras on Margarita. In each of these trips, that of 1901 and that of 1903, between 3000 and 4000 specimens were secured, all of which have been distributed among the leading herbariums in this country and abroad, the most nearly complete set being at the Gray herbarium of Harrard university. It is unfortunate that the first collection was distributed in an incomplete and poorly identified condition but it is hoped that the present listing of the species together with the citation of the collection numbers and dates may tend to correct any previous errors. In the main the identifications hare been carried on at the Gray herbarium. Mr. O. O. Miller of the party of 1901 assisted in the identifications of the collection of that year. 'The rest of the work, in which I have been generously assisted by the staff of the Gray herbarium, has derolved upon me. Mr. Oakes Ames identified part of the Orchidaceae; Dr. Carl Mez, part of the Bromeliaceae; Dr. Casimir de Candolle, the Piperaceae; Professor Radlkofer, one of the Sapin- 
aceac; and Mr. IV. R. Maxon has revised the list of ferns. Professor I. Urban has made some corrections in my identifications and has published several new species based upon my plants. The collection of fungi and of algae marle by Dr. A. F. Blakeslee has been identified by Professor W. G. Farlow and is deposited in the Cryptogamic herbarium of Harvard university. 'This collection is not included in the following list of Margaritan plants.

To all these who have so generously assisted me and especially to Professor B. L. Robinson under whose supervision this work has been done and to Miss M. A. Day, librarian of the Gray herbarium, who has verified the references and otherwise assisted in the bibliographical work, many thanks are due.

In working out the identifications of the plants listed in the following catalogue many difficulties were encountered, chief among them being the great lack of South American plants for comparison, and the uncertain condition of many South American genera and species. These difficulties have been overcome to some extent by the assistance, already referred to, of specialists on certain groups. The bulk of the plants, however, remained in groups which have not been revised for some time.

The citations to be found in the catalogue, consist of the original references to the names which are used, a reference to the earliest use of the specific name, and in addition reference to either or both Grisebach's Florn of the British West Indies and Martius' Flora Brasiliensis, as well as a reference to some published illustration, and when possible a citation of monographs of the various groups. These are the works which have been used in conjunction with herbarium specimens to make the identifications, to determine the character of the species. From these my idea of the species was obtained and for that reason it seems desirable to cite them in this list. In addition to these references I have also given the occurrence of the species in Venezuela as a whole, this being ascertained by reference both to publications and to specimens. All speeimens here reported and collceted by Fendler, by Rusby and Squire, by Robinson and Lyon, by Birschel, and by A. H. Moore have been personally examined by me and compared with my own material. Other references than those of species to Venezuela are taken from publications and are given for only what they may be worth under such circumstances. The reference to Ernst with some page number is to Ernst's Estudios sobre la flora y fauna. 
After the citation of Venezuelan plants, I lave given reference to the occurrence of the species in Porto Rico as found in Urban's Flora portoricensis. These citations are based upon Professor Urban's comparison of my plants with his so that the distribution of the plants in these two localities is made certain. 'The last note after a species is in regard to its general distribution.

The arrangement of the families is as in Engler and Prantl's Pflanzenfamilien, and the genera and species are alphabetically arranged in their respective groups.

While it is to be regretted that each species coukl not be subjected to the critical examination of a specialist it is to be hoped that this list in connection with the distributed plants may be of value in future work upon the flora of Venezuela.

\section{PTERIDOPHYTA. ${ }^{1}$}

\section{GLEICHENIACEAE.}

\section{DicRaNopteris Bernh.}

D. Flexuost (Schrad.) Lnderw., Bull. Torr. bot. club, vol. 34, p. $25+(1907)$. Mertensia flexuosa Schrad., Goett. Anz., p. S63 (1S24). M. rigida Kunze, Linnaea, vol. 9, p. 16 (1834). Gleichenia flexuosa Mett., Ann. Lug. Bat., vol. 1, p. 50 (1S63). G. rigida Bommer \& Christ, Bull. soc. bot. Belg., vol. 35, p. 174 (1S96).- San Juan Mt., alt. $700 \mathrm{~m}$., July 16, Johnston, no. 180 . Tropical America.

\section{CYATHEACEAE.}

Crathea Sm.

C. tenera (J. Sm.) Hook. ex Moore, Ind. Fil., p. $27 t$ (1861). Alsophila tenera J. Sm., Lond. Journ. bot., vol. 1, p. 666 (1S42), nomen nudum. A. tenera J. Sm. ex Hook., Sp. Fil., vol. 1, p. 49 (1S+6).- Rio Asuncion, alt. $450 \mathrm{~m}$., Johnston, no. 143, July 29. Also on Trinidad and several of the West Indies.

\section{Henitela R. Br.}

H. Grandifolda (Tilld.) Spreng. Syst, rol. 4, p. 125 (1827). Cyathea grandifolia Willd., Sp. pl., vol. 5, p. 490 (1S10)._- El Valle,

1 The identifications in this group are as revised by Mr. W. R. Maxon, who has also added the synonymy and the notes in regard to the species. 
Miller \& Johnston, no. 164, in part, Aug. 16; San Juan Mit., alt. 500 m., Johnston, no. 191, July 16. Porto Rico: Urban, Symb. Ant., vol. 4, p. 10 (1903). Tropical America.

\section{POLYPODIACEAE.}

\section{Elaphoglossum Schott.}

E. tovarense (Mett.) Moore, Ind. Fil., p. 369 (1S62), name only. Aehrostichum tovarense Moritz ex Eaton, Mem. Amer. acad. arts and sci., (new series) vol. S, pt. 2, p. 194 (1S60), name only; Kuhn, Linnaea, vol. 36, p. 60 (1869).- Juan Griego trail, alt. 450 m., Johnston, no. 146. Known also from Mexico and Central America.

\section{Monogrania Commerson.}

M. Livearifolia Desv. Mag., Gesellsch. nat. Fr. Berlin, vol. 5, p. 302, pl. 7, fig. 5 (1811). Pleurogrammc immersa Fée, 3me Mém., p. 37, pl. 4, fig. 5 (1851-52). Monogramme immersa Hook., Sp. Fil., vol. 5, p. 125 (1864).- San Juan Mt., alt. 600 m., Johnston, no. 1S6, July 2. West Indies and northern South America.

\section{Camprloneurum Presl.}

C. Phrluttidis (L.) Presl, Tent. Pterid., p. 190, pl. 7, fig. 18 (1836). Polypodium phyllitidis L., Sp. pl., vol. 2, p. 1083 (1753); Urb., Symb. Ant., vol. 4, p. 57 (1903), where reported as occurring on Margarita Island.-El Valle, Miller \& Johnston, no. 165, July 30; San Juan Mt., alt. 400 m., Johnston, no. 151, July 27. Generally distributed throughout tropical America.

\section{Goniophlebium Presl.}

G. Chyoodes (Spreng.) Fée, Gen. Fil., p. 255 (1850-52). Polypodium chnoodes Spreng., Nene Entdeck., vol. 2, p. 6 (1822).- San Juan Mt., alt. 600 m., Johnston, no. 193, July 2. Tropical America, commonest in the West Indies.

G. LORiceuri (L.) J. Sm. ex Hook., Gen. Fil., under pl. 51 (1S10). Polypodium 7oriceum L., Sp. pl., vol. 2, p. 1086 (1753).- Rio Asuncion, alt. 500 m., Johnston, no. 169, Aug. 12-15. Tropical America generally. Variable.

G. Pilosellondes (L.) J. Sm. ex Hook., Gen. Fil., under pl. 51 
(1810). Polypodium piloselloides L., Sp. pl., vol. 2, p. 1083 (1753). Lopholepis piloselloides J. Sm. in Seem., Bot. roy. Herald, p. 229 (1S.54).- Juan Griego trail, alt. 500 m., Johnston, no. 150, July 31. Tropical America.

G. Neriffolium (Schkuhr) Hook., Gen. Fil., pl. 70 B (1S41). Polypodium neriifolium Schkuhr, Krıpt. Gewächse, vol. 1, p. 14, pl. 15 (1S06).-San Juan MI., alt. 700 m., Johnston, no. 176, Aug. $2 S$. Tropical America.

G. racciniffolium (Langsd. \& Fisch.) J. Sm. ex Hook., Gen. Fil., under pl. 51 (1S40). Polypodium vacciniifolium Langsd. \& Fisch., Ic. Fil., vol. S, pl. 7 (1S10).—El Valle, Miller \& Johnston, no. 159, July 31. Tropical America.

\section{Phlebodium J. Sm.}

P. aurecir (L.) J. Sm., Journ. bot., vol. 4, p. 59 (1St1). Polypodium aureum L., Sp. pl., rol. 2, p. 1087 (1753).- Rio Asuncion, alt. $500 \mathrm{~m}$., Johnston, no. 170, Aug. 12-15. Widely distributed in tropical America.

\section{Phymatodes Presl.}

P. xematorhizor (D. C. Eaton) Underw. ex Maxon, Contrib. U. S. nat. herb., vol. 10, p. 493 (1908). Polypodium nematorhizon D. C. Eaton, Bot. gaz., vol. 3, p. 90 (1S7S). - Juan Griego trail, alt. $500 \mathrm{~m} .$, Johnston, no. 147, Aug. 12-15. Known hitherto only from Trinidad.

P. Prominula Maxon, Contrib. U. S. nat. herb., rol. 10, p. 501 (1908). ? Polypodium salicifolium Willd., Sp. pl., vol. 5, p. 149 (1810), not Vahl (1S0\%).- San Juan Mt., alt. 500 m., Johnston, no. 155 (trpe). Tropical America.

\section{Polypodiug L.}

P. Jubaeforme Kaulf., Flora, vol. 6, pt. 1, p. $36 \pm$ (1823). Polypodium saccatum Fée, Gen. Fil., p. 239 (1S50-52).- San Juan Mt., alt. $600 \mathrm{~m}$., Johnston, no. 164, July 2. The West Indies and Panama to Brazil.

P. pectinatum L., Sp. pl., rol. 2, p. 10S5 (1753).—El Talle, Miller \&. Johnston, no. 163; Juan Griego trail, alt. 450 m., Johnston, no. 167, July 19. Tropical America generally. 
P. Polypodioldes (L.) A. S. Hitchcock, Rep. Mo. bot. garden, vol. 4, p. 156 (1893). Acrostichum polypodioides L., Sp. pl., vol. 2, p. 1065 (1753). Polypodium incanum Sw., Prod. veg. Ind. Occ., p. 131 (1788).- San Juan Mt., alt. 450 m., Johnston, no. 157, July 6. Southern and central United States to Argentina.

\section{Xiphopteris Kaulf.}

X. serrulata (Sw.) Kaulf., Enum. Fil., pp. \$5, 300 (1824). Acrostichum serrulatum Sw., Prod. veg. Ind. Occ., p. 128 (1788). Polypodium serrulatum Mett., Fil. hort. Lips., p. 30 (1S56), not Sw. (1800). Xiphopteris extensa Fée, 11 me Mém., p. 14, pl. 19, fig. 3 (1866), not Polypodium extensum Forst. (1786), Presl (1825), nor Fée (1869).Juan Griego trail, alt. 450 m., Johnston, no. 144, Aug. 12-15. Tropical America generally. Also occurs in Africa.

\section{Adiantopsis Fée.}

A. Radiata (L.) Fée, Gen. Fil., p. 145 (1850-52). Adiantum radiatum L., Sp. pl., vol. 2, p. 1094 (1753).-San Juan Mt., alt. 400 m., Johnston, no. 18t, July 6 . Not uncommon in the American tropics.

\section{Adiantum L.}

A. tetraphillum H. \& B. ex Willd., Sp. pl., vol. 5, 441 (1810); Urb., Symb. Ant., vol. 4, p. 45 (1903), where reported as occurring on Margarita Island.-El Valle, Miller \& Johnston, no. 166, July 25; Juan Griego trail, alt. 300 m., Johnston, no. 162. Tropical America.

\section{Ceropteris Link.}

C. Calonelaena (L.) Link, Fil. sp. hort. bot. Berol., p. 141 (1S41). Acrostichum calomelanos L., Sp. pl., vol. 2, p. 1072 (17533). Gymnogramma calomelanos Kaulf., Enum. Fil., p. 76 (1S24).- San Juan Mt., alt. 550 m., Johnston, no. 182, July 19. Tropical America. Africa. Escaped from cultivation in Luzon.

C. tartarea (Cav.) Link, Fil. sp. hort. bot. Berol., p. 142 (1St1). Acrostichum tartareum Cav., Descr. pl., p. 242 (1802). Gymnogramma tartarea Desv., Mag., Gesellsch. nat. Fr. Berlin, vol. 5, p. 305 (1811).- San Juan Mt., alt. 795 m., Johnston, no. 181, Aug. 28. 'Tropical America; uniformly at a higher elevation than the preceding. 


\section{Pteridium Scop.}

P. Caudatuil (L.) Maxon, Proc. U. S. nat. mus., vol. 23, p. 631 (1901). Pteris caudata L., Sp. pl., vol. 2, p. 1075 (1753). Pteris aquilina, var. caudata Hook., Sp. Fil., vol. 2, p. 196 (1S58)._- San Juan Mt., alt. 790 m., Johnston, no. 177, Aug. 2S. Tropies.

\section{Asplexium L.}

A. A '́scissuru Willd., Sp. pl., vol. 5, p. 321 (1S10). Asplenium laetım Schkuhr, Krypt. Gewächse, vol. 1, p. 65, p] 70 (1S09), not Sw. (1S06). A. Schkuhrianum Presl, Tent. Pterid., p. 107 (1836). A. firmum Kunze, Bot. Zeit., vol. 3, p. 283 (1845).- Juan Griego trail, alt. $450 \mathrm{~m}$,, Johnston, no. 154, July 29. Tropical America generally.

A. Cirrhatum Rich. ex Willd., Sp. pl., vol. 5, p. 321 (1S10). Asplenium rhizophorum (simple-pinnate form) of most authors.- Juan Griego trail, alt. 450 m., Johnston, no. 142, July 31. Tropical America; commonest in the West Indies.

A. Cristatim Lam., Encyc., vol. 2, p. 310 (17S6). Asplenium cicutarium Sw., Prod. reg. Ind. Occ., p. 130 (17SS); Urb., Symb. Ant., vol. 4, pp. 3S, 39 (1903), where reported as occurring on Margarita Island.-EI Valle, Miller \& Johnston, no. 162, Aug. 2; Juan Griego trail, alt. $450 \mathrm{~m}$., Johnston, no. 161, July 22. American tropics; reaching its best development in the West Indies.

A. Cuneatcm Lam., Encyc., vol. 2, p. 309 (17S6).- Juan Griego trail, alt. $450 \mathrm{~m}$., Johnston, no. 172, July 31. Tropical America, the typical form being West Indian. Ascribed also to the tropies of the Old World.

A. Salicifolium L., Sp. pl., vol. 2, p. 1080 (1753). Asplenium auriculatum and A. semicordatum of authors, as to West Indian specimens (see Maxon, Contrib. U. S. nat. herb., vol. 10, p. 476 (1908) for synonymy). - Juan Griego trail, alt. $450 \mathrm{~m}$., Johnston, no. 16S, July 31. Tropical America.

A. serratum L., Sp. pl., vol. 2, p. 1079 (1753).-San Juan Mt., alt. $400 \mathrm{~m}$., Johnston, no. 141, July 6. American tropies. Common.

\section{BlechNum L.}

B. occidentale L., Sp. pl., vol. 2, p. 1077 (1753); Urb., Symb. Ant., vol. 4, p. 39 (1903), where reported from Margarita Island.- 
El Valle, Miller \& Johnston, no. 155, Aug. 2; San Juan Mt., alt. 400 m., Johnston, no. 160, July 16. American tropics; ubiquitous.

\section{Diplazicui Sw.}

D. arboreum (Willd.) Presl, Tent. Pterid., p. 114 (1S36), not of most authors. Asplenium arboreum Willd., Sp. pl., vol. 5, p. 320 (1S10). A. Shepherdi Spreng., Nov. act. acad. Caes. Leop., vol. 10, p. 231, pl. 17, figs. 5 and 6 (1S21). Diplazium Shepherdi Link, Hort. Berol., vol. 2, p. 70 (1833).- Juan Griego trail, alt. 450 m., Johnston, no. 158, July 29. Tropical America.

D. Crenulatum Liebm. Vid. selsk. skr., ser. 5, vol. 1, p. 254 (1S49). Earlier synonymy much confused: the Linnaean name not available. Referred under D. striatum (L.) Presl by Urban (Symb. Ant., vol. 4, p. 33 (1903) as occurring on Margarita Island.-El Valle, Miller \& Johnston, no. 161, Aug. 16; Juan Griego trail, alt.. 450 m., Johnston, no. 153, July 29. Tropical America.

D. Grandifolium Sw. in Schrad., Journ. bot., 1800, pt. 2, p. 62 (1501). Asplenium grandifolium. Sw., Prod. veg. Ind. Occ., p. 130 (17SS). - Juan Griego trail, alt. 450 m., Johnston, no. 159, July 29. Tropical America.

\section{DRYOPTERIS Adans.}

D. ampla (H. \& B.) Kitze., Rer. gen., vol. 2, p. $\$ 12$ (1S91). Polypodium amplum H. \& B. ex Willd., Sp. pl., vol. 5, p. 207 (1S10).El Valle, Miller \& Johnston, no. 169, Aug. 2; Juan Griego trail, alt. $450 \mathrm{~m}$., Johnston, no. 187 in part, July 29. Tropical America. Florida.

D. Johnstoni Maxon, Contrib. U. S. nat. herb., vol. 10, p. 498 (1908).- Juan Griego trail, alt. 450 m., Johnston, no. 192, July 22 (type). Known also from Trinidad, Jenman; Fendler, no. 54.

D. MEgalodus (Schkuhr) Urb., Symb. Ant., vol. 4, p. 21 (1903). Polypodium megalodus Schkuhr, Krypt. Gewächse, vol. 1, p. 24, pl. 19b (1S06).- El Valle, Miller \& Johnston, no. $16 t$ in part. 'Tropical America.

D. Parasitica (L.) Kítze., Rev. gen., vol. 2, p. 811 (1S91). Polypodium parasiticum L., Sp. pl., vol. 2, p. 1090 (1753). Nephrodium parasiticum Desr., Mém. soc. Linn. Paris, vol. 6, p. 260 (1S27). Polypodium molle Jacq., Coll. bot., vol. 3, p. $1 S S$ (17S9), not Schreb. (1771), nor All. (1755). Nephrodium molle R. Br., Prod. fl. Nov. 
Holl., p. 149 (1S10).- Juan Griego trail, alt. 450 m., Johnston, no. 194, July 19. Tropical and subtropical regions of both hemisphercs. Rare in the southern United States.

D. pubescens (L.) Ktze., Rev. gen., vol. 2, p. $\$ 13$ (1S91). Polypodium pubescens L., Syst. nat., ed. 10, vol. 2, p. 1327 (1759).Juan Griego trail, alt. 450 m., Johnston, no. 195, Aug. 12-15. The specimens are broader and less produced than the typical Jamaican form. West Indies.

D. Mercurit (A. Br.) Hieron., Hedwigia, vol. 46, p. 335, pl. 5, fig. 9 (1907). Aspidium Mercurii A. Br. ex Christ., Bull. herb. Boiss., ser. 2, vol. 6, p. 58 (1906), name only.-Juan Griego trail, alt. $450 \mathrm{~m} .$, Johnston, no. 190, July 29. Determined by Christensen, who attributes the species also to Mexico, Costa Rica, Colombia, and Ecuador.

[D. tetragona (Sw.) Urb., Symb. Ant., vol. 4, p. 20 (1903). Polypodium tetragonum Sw., Prod. veg. Ind. Occ., p. 132 (17SS). - Stated by Urb., l. c., to grow on Margarita Island, a report based upon material collected by Miller \& Johnston in 1901.]

D. villosa (L.) Ktze., Rev. gen., vol. 2, p. 314 (1S91). Polypodium villosum L., Sp. pl., vol. 2, p. 1093 (1753). Nephrodium villosum Prest, Rel. Haenk., vol. 1, p. 38 (1S25). Juan Griego trail, alt. $450 \mathrm{~m}$., Johnston, no. 187 in part, July 29. Tropical America.

\section{Menisciuir Schreb.}

II. Reticulatui (L.) Sw. in Schrad., Journ. bot., 1801, pt. 1, p. 274 (1803). Polypodium reticulatum L., Syst. nat., ed. 10, vol. 2, p. 132.5 (1759). Nephrodium reticulatum Keyserling, Pol. Crath. herb. Bung., p. 49 (1S73). Dryopteris reticulata Urb., Symb. Ant., rol. 4, p. 22 (1903).— El Valle, Miller \& Johnston, no. 156, Aug. 16; Juan Griego trail, alt. 450 m., Johnston, no. 166, July 22. Tropical America.

\section{Olfersia Raddi.}

O. cervina (L.) Kunze, Flora, vol. 7, pt. 1, p. 312 (1824). Osmunda cervina L., Sp. pl., vol. 2, p. 1065 (1753). Polybotrya cervina Kaulf., Enum. Fil., p. 55 (1S24).- Juan Griego trail, alt. 450 m., Johnston, no. 152, July 31. Tropical America.

\section{Tectaria Cav.}

T. heracleifolia (Willd.) Underw., Bull. Torr. bot. club, vol. 33, p. 200 (1906). Aspidium heracleifolium Willd., Sp. pl., vol. 5, p. 217 
(1810). Aspidium trifoliatum of authors, in part, not Polypodium trifoliatum L., based on Plumier's plate 148, see Underw., l. c., p. 199-200.- El Valle, Miller \& Johnston, no. 168, Aug. 2. Reported by Urb. (Symb. Ant., vol. 4, p. 23), under the name of Aspidium trifoliatum, as occurring upon Margarita Island, his determination being based upon some of Miller \& Johnston's material of 1901. Tropical America generally.

T. Plantaginea (Jacq.) Maxon, Contrib. U. S. nat. herb., vol. 10, p. 494 (190S). Polypodium plantagineum Jacq., Coll. bot., vol. 2, p. 104, pl. 3, fig. 1 (1788). Aspidium plantagineum Griseb., Abh. kön. Gesellsch. Wiss. Göttingen, vol. 7, p. $2 \$ 6$ (1S57).- Juan Griego trail, alt. 500 m., Johnston, no. 14S, July 31. Tropical America. Variable.

T. Purdaei (Jenman) Maxon, Contrib. U. S. nat. herb., vol. 10, p. 494 (1908). Aspidium Purdiaei Jenman, Gard. chron., ser. 3, vol. 22, p. 282 (1S97). Nephrodium Sherringiae Jenman, Journ. bot., vol. 25, p. 99 (1887), not $N$. Sherringii Jenman (1879). Aspidium psammisorum C. Chr., Ind. Fil., p. $\$ 9$ (1905).- El Valle, Miller \& Johnston,'no. 15S, Ang. 10; Juan Griego trail, alt. 450 m., Johnston, no. 173, July 31 . Known also from Trinidad.

\section{Oleandra Cav.}

O. nodosa (Willd.) Presl, Tent. Pterid., p. 75 (1836). Aspidium nodosnm Willd., Sp. pl., vol. 5, p. 211 (1S10).-Juan Griego trail, alt. $300 \mathrm{~m}$., Johnston, no. 189, July 29. American tropics. Ascribed also to Africa.

\section{Dennstaedta Bernh.}

D. ordinata (Kaulf.) Moore, Ind. Fil., p. 306 (1861). Dicksonia ordinata Kaulf., Enum. Fil., p. 226 (1S21)._El Valle, Miller \& Johnston, no. 160, Aug. 16; Juan Griego trail, alt. 450 m., Johnston, no. 185, July 29. Widely distributed in tropical America.

\section{Nephrolepis Schott.}

[N. exaltata (L.) Schott, Gen. Fil., pl. 3 (1834). Polypodium exaltatum I., Syst. nat., ed. 10, vol. 2, p. 1326 (1759).-- Referred by Urban, Symb. Ant., vol. 1, p. 27, to Margarita.]

N. Pectinata (Willd.) Schott, Gen Fil., under pl. 3 (18.54). Aspidium pectinatum Willd., Sp. pl., vol. 5, p. 223 (1810).- San Juan Mt., alt. 790 m., Johnston, no. 149, July 16. Tropics. 
N. Rivularis (Tahl) Mett. ex Krug in Engl., Bot. jahrb., rol. 24, p. 122 (1S97). Polypodium rivulare Vahl, Ecl. Anı., vol. 3, p. 51 (1S07). Aspidium sesquipedale Willd., Sp. pl., vol. 5, p. 230 (1S10). Nephrolepis sesquipedalis Presl, 'T'ent. Pterid., p. 79 (1S36).-El Valle, Miller \& Johnston, no. 167, Aug. 2; Juan Griego trail, alt. 450 m., Johnston, no. 163, July 22. Tropical America.

\section{HYMENOPHYLLACEAE.}

\section{Hruenophyllum J. E. Sm.}

H. Polyaxthos Sw. in Schrad., Journ. 1S00, pt. 2, p. 102 (1S01). Trichomanes polyanthos Sw., Prod. reg. Ind. Occ., p. 137 (17SS).El Valle, Miller \& Johnston, no. 157, July 31; Juan Griego trail, alt. 500 m., Johnston, no. 165, July 2. Porto Rico: Urban, Srmb. Ant., rol. 4 , p. 8 . Widespread in the tropics, the typical form being West Indian.

H. ciliatum Sw. in Sclirad., Journ. bot., 1S00, pt. 2, p. 100 (1S01) et Fl. Ind. Occ., vol. 3, p. 1753 (1S06).-This species is reported by Urban (Symb. Ant., vol. 4, p. 9) as occurring on Margarita, this being his determination of Miller \& Johnston's material of 1901. The specimen in question may possibly be referable, howerer, to Trichomanes.

H. Kohautianum Presl, Hymenoph., p. 32 and p. 56 (1St3). Referred by Urban to Margarita. See note on preceding species.

\section{Trichomanes L.}

T. AlAtum Sw. in Schrad., Journ. bot., 1800, pt. 2, p. 97 (1801).Rir Asuncion, alt. 450 m., Johnston, no. 145, July 29. Tropical America: the type from Jamaica. Variable.

T. Crisply L., Sp. pl., vol. 2, p. 1097 (1753).-San Juan Mt., alt. $600 \mathrm{~m} .$, Johnston, no. 171, July 2. Tropical America.

T. membraxacecir L., Sp. pl., vol. 2, p. 1097 (1753).- Juan Griego trail, alt. 450 m., Johnston, no. 15, Aug. 12-15. 'Tropical America.

'T. Polypodioides L., Sp. pl., vol. 2, p. 1095 (1753). T. sinuosum Rich. ex Willd., Sp. pl., vol. 5, p. 502 (1S10); Hook. \& Grer., Ic. Fil., vol. 1, pl. 13 (1831).- Rio Asuncion, alt. 450 m., Johnston, no. 175, Aug. 12-15. Tropical America. 


\section{LICOPODIACEAE.}

\section{LyCOPODIUM L.}

L. Funiforme Bory in Brongn., Vég. foss., vol. 2, p. 10 (1S37); Spring, Mém. acad. Brux., vol. 15, pt. 6, p. 50 (1St2), vol. 24, p. 22 (1S49).- San Juan Mt., alt. 600 m., July 2, Johnston, no. 179. Tropical America; commonest in the West Indies.

L. Taxifolium Sw., Prod. reg. Ind. Occ., p. 138 (17SS). - San Juan Mt., alt. 600 m., Johnston, no. 156, July 2. Tropical America.

\section{SPERMATOPHYTA.}

\section{HYDROCHARITACEAE.}

Thalassia Banks.

T. Testudinum Kon. in Kon. \& Sims, Ann. bot., vol. 2, p. 96 (1S06); Griseb., Fl. Brit. W. Ind., p. 507.-Juan Griego, Ernst. Jamaica and Caribbean Is.

\section{GRAMINEAE.}

\section{ANThEphora Schreb.}

A. hermaphrodta (L.) K'tze., Rev. gen., vol. 2, p. 759 (1S91); Urb., Symb. Ant., vol. 4, p. S0. Tripsacum hermaphroditum L., Syst. nat., ed. 10, vol. 2, p. 1261 (1759). A. clegans Schreb., Beschr. Gräs., vol. 2, p. 105, pl. 44 (1810).-El Valle, Miller \& Johnston, no. 173, July 1S. Venezuela: near Villa del Pao, HBK. Nov. gen. et sp. vol. 1, p. 116; Porto Rico: Urban, l.c. Widely distributed in tropical America.

\section{Cenchrus L.}

C. Echinatus L., Sp. pl., vol. 2, p. 1050 (1753); Griseb., Fl. Brit. IV. Ind., p. 5.56; Mart., Fl. Bras., vol. 2, pt. 2, pl. 43; Cav., Ic. pl., p. 462.- Santa Ana, Ernst. Tenezuela: near Cumaná, HBK. Nov. gen. et sp., vol. 1, p. 114; Loefling, Reise nach den spanischen Ländern, 152-153. Widely distributed in tropical comtries.

C. viridis Spreng., Syst., vol. 1, p. 301 (1\$25); Doell in Mart. Fl. Bras., vol. 2, pt. 2, p. 309; Lrb., Symb. Ant., vol. 4, p. 97.- El Valle, Willer \& Johnston, no. 186 and 179. Tropical America. 


\section{DActrlocteniem Willd.}

D. Aegrptium (L.) Richter, Pl. Eu., rol. 1, p. 68 (1S90); Urb., Symb. Ant., vol. 4, p. 104. Cynosurus aegyptius L., Sp. pl., 1. 72 (1753). Eleusine acgyptia Desf., Fl. Atl., vol. 1, p. 85 (1798). D. acgyptiacum Willd., Enum hort. Berol., p. 1029 (1809).-El Valle, Miller \& Johnston, no. 174, July S. Widely distributed in tropical countries.

\section{Eragrostis Host.}

E. ciliaris (L.) Link, Hort. Berol., vol. 1, p. 192 (1S2̄); Griseb., Fl. Brit. W. Ind., p. 532; Doell in Mart., Fl. Bras., rol. 2, pt. 3, p. 155; Lrb., Symb. Ant., vol. 4, p. 10S. Poa ciliaris L., Syst. nat., ed. 10, rol. 2, p. S75 (1759).- El Valle, Miller \& Johnston, no. 175, Aug. 15. Venezuela: Ernst in Seem., Journ. bot., vol. 5, p. 29.j (1S67). Widely distributed in tropical countries.

\section{GrNerium H. \& B.}

G. Sagittatur (Aubl.) Beauv., Agrost., p. 135 (1S12); Crb., Symb. Ant., vol. 4, p. 105. Saccharum sagittatum Aubl., Pl. Guian., vol. 1, p. 50 (1775). G. saccharoidcs H. \& B., Pl. Aequin., vol. 2, p. 112, pl. 11 (1809). - EI Valle, Hiller \& Johnston, no. 193, July 30. Venezuela: near Cumaná, HBK. Nor. gen. et sp., rol. 1, p. 149. Widely distributed from Mexico and Cuba to Brazil.

\section{IchNaNTHUs Beauv.}

I. Pallexs (Sw.) Munro ex Benth., Fl. Hongk., p. 414 (1S61); Doell in Mart., Fl. Bras., vol. 2, pt. 2, p. 290; Urb., Symb. Ant., rol. 4, p. 94. Panicum pallens Sw., Prod. veg. Ind. Occ., p. 23 (17SS); Steud., Gram., p. 93.-San Juan Mt., alt. 600 m., Johnston, no. 208, July 16. Venezuela: Ernst, Sobre la flora y fauna, p. 223. American tropics and East Indies.

\section{LEPTOCHLOA' Beauv.}

L. Filiformis (Lam.) Beauv., Agrost., p. 71 (1S12); Doell in Mart., Fl. Bras., vol. 2, pt. 3, p. 93; Urb., Symb. Ant., vol. 4, p. 10t. Festuca filiformis Lam., Ill., vol. 1, p. 191 (1791). L. mucronaia Kth., Rer. Gram., rol. 1, p. 91 (1829).-EI Valle, Miller \& Johuston, no. 16, July 30. Venezuela: Ernst in Seem., Journ. bot., rol. 5, p. 295 (1S67) as L. mucronata. Distributed generally in tropical countries. 
L. virgata (L.) Beaur., Agrost., p. 71 (1812); Griseb., Fl. Brit. IV. Ind., p. 53S; Urb., Symb. Ant., vol. 4, p. 105. Cynosurus virgatus L., Syst. nat., ed. 10, vol. 2, p. $\$ 76$ (1759).—El Valle, Miller \& Johnston, no. 197, July 26. Widely distributed in tropical America.

\section{Olyra L.}

O. Latirolia L., Syst. nat., ed. 10, vol. 2, p. 1261 (1759); Griseb., Fl. Brit. IV. Ind., p. 535.- Juan Griego trail, alt. 450 m., Johnston, no. 314, July 29. Distributed generally in tropieal America.

\section{Panicum L.}

P. Divaricatum L., Syst. nat., ed. 10, vol. 2, p. S71 (1759); Griseb., Fl. Brit. W. Ind., p. 551.-El Valle, Miller \& Johnston, no. 1S4, July 30. Venezuela: near Maypurea, HBK., Nov. gen. et sp., vol. 1, p. 101. Porto Rico: Urb., Symb. Ant., vol. 4, p. 91. American tropies.

P. insulare (L.) G. F. IT. Mey., Prim. Esseq., p. 60 (1S1S); Urb., Symb. Ant., vol. 4, p. S6. Andropogon insulare L., Syst. nat., ed. 10, vol. 2, p. 1304 (1759).- El Valle, Miller \& Johuston, no. 1S3, July 20. American tropies and subtropics.

P. Latifolium L., Sp. pl., vol. 1, p. 5 S (1753).—Juan Griego trail, alt. 450 m., Johnston, no. 196, Aug. 12-15. Venezuela: Ernst, Sobre la flora y fauna, p. 223. Distribution general in North America and West Indies.

P. maximus Jacq., Ic. pl. rar., vol. 1, pl. 13 (17S1); Griseb., Fl. Brit. W. Ind., p. 549; Doell in Mart., Fl. Bras., vol. 2, pt. 3, p. 202.El Valle, Miller \& Johnston, no. 177, July 7. Venezuela: Ernst, Sobre la flora y fauma, p. 223. Porto Rico: Urb., Symb. Ant., vol. 4, p. 89. Distribution general in tropical America.

P. Paniculatum (L.) Nash, Bull. Torr. bot. club, rol. 30, p. 381 (1903); Urb., Symb. Ant., vol. 4, p. 90. Paspalum paniculatum L., Syst. nat., ed. 10, vol. 2, p. 855 (1759). - El Valle, Miller \& Jolnston, no. 180, July 26. Distribution general in tropical Ameriea.

P. Prostratum Lam., Illustr., vol. 1, p. 171 (1791); Griseb., Fl. Brit. W. Ind., p. 546. El Talle, Miller \& Johnston, no. 171, Aug. 6. Tenezuela: Ernst, Sobre la flora y fauna, p. 223. Porto Rico: Urb., Symb. Ant., vol. 4, p. 88 . Widely distributed in tropical countries.

P. sanguinale L., var. Horizontale (Willd.) Schweinf., Bull. 
herb. Boiss., vol. 2, app. 2, p. 18 (1894); Urb., Symb. Ant., vol. 4, p. S6. Digitaria horizontalis Willd., Enum., vol. 1, p. 92 (1S09).El Valle, Miller \& Johnston, no. 185, July 10. Distribution general in tropics.

\section{Paspalum L.}

P. conjug.ıtur Berg., Act. Helv., vol. S, p. 129, pl. S (1772); Griseb., Fl. Brit. W. Ind., p. 541.-El Valle, Miller \& Johnston, no. 178, July 26; Juan Griego trail, Johnston, no. 198, Aug. 12-15. Venezuela: near San Fernando de Atabapo and near Bordones, Cumaná, etc., HBK., Nov. gen. et sp., vol. 1, p. 91. Porto Rico: Urb., Symb. Ant., vol. 4, p. $\$ 1$. Common in tropical countries.

P. compressum (Sw.) Rasp., Ann. sci. nat., ser. 1, rol. 5, p. 301 (1825); Griseb., Fl. Brit. W. Ind., p. 541; Urb., Symb. Ant., vol. 4, p. 84. Milium compressum Sw., Prod. reg. Ind. Occ., p. 24 (1788). Paspalum platicaulon Poir., Encyc., rol. 5, p. 34 (1804).- Juan Griego trail, Johnston, no. 197, Aug. 12-15. Venezuela: Ernst, Sobre la flora y fauna, p. 223. Distribution general in tropical America.

\section{Pharus P. Br.}

P. LAtifolius L., Syst. nat., ed. 10, vol. 2, p. 1269 (1759); Griseb., Fl. Brit. W. Ind., p. 536; Urb., Symb. Ant., vol. 4, p. 99.-El Valle, Miller \& Johnston, no. 170, Aug. 2; Juan Griego trail, alt. 400 m., Johnston, no. 200, Aug. 12-15. Venezuela: Ernst, Sobre la flora y fauna, p. 223. Distribution general in tropical America.

\section{SACChaRUit L.}

S. officinaruar L., Sp. pl., rol. 1, p. 54 (1753); Griseb., Fl. Brit. II. Ind., p. 561.-Cultivated in El Talle and in Asuncion.

\section{Setaria Beauv.}

S. glaUCA (L.) Beauv., Agrost., p. 51 (1812); U'rb., Symb. Ant., vol. 4, p. 95 . Panicum glancum L., Sp. pl., vol. 1, p. 56 (17j3). Chaetochloa glauca Scribn., U. S. dept. agr., div. agrost., bull. no. 4 , p. 39 (1S97).-El Valle, Miller \& Johnston, no. 181, July 26. Venezuela: Ernst, Sobre la flora y fauna, p. 223. Tropical countries.

Sporobolus R. Br.

S. domingensis (Trin.) Kunth, Enum. pl., rol. 1, p. 214 (1833); Griseb., Fl. Brit. IV. Ind., p. 533. Tilfa domingensis Trin. in Spreng., 
Nene Entcleck., vol. 2, p. 59 (1S21).— Pt. Piedras, Johnston, no. 325, Ang. 6, and at Laguna Chica, Johnston, no. 327, Aug. 10, a much reduced form. Venezuela: Ernst, Sobra la flora y fauna, p. 223. Jamaica and Haiti.

S. vinglyicus (L.) Kunth, Rev. Gram., vol. 1, p. 67 (1829); Griseb., Fl. Brit. Wr. Ind., p. 533. Agrostis virginica L., Sp. pl., vol. 1, p. 63 (1753).- Pt. Mosquito, Johnston, no. 326, Aug. 10. Venezuela: 'Tortuga, Emst; Los Roques, Ernst. Widely distributed in tropical countries.

\section{'Tragus Haller.}

T. Racenrosus (L.) Scop., Introd., p. 73 (1777); Doell in Mart., Fl. Bras., vol. 2, pt. 2, p. 122. Cenchrus racemosus L., Sp. pl., vol. 2, p. 1049 (1753).- Reported by Urban (Symb. Ant., vol. 4, p. S0) as occurring on Margarita, a determination based on Miller \& Johnston's material of 1901.

\section{ZEA L.}

Z. Mars L., Sp. pl., vol. 2, p. 971 (1753); Griseb., Fl. Brit. IT. Ind., p. 561; Doell in Mart., Fl. Bras., vol. 2, pt. 2, p. 31.-Cultivated in El Valle and Asuncion.

\section{CYPERACEAE.}

\section{Crperus L.}

C. Distaxs L. f., Suppl., p. 103 (1781); Griseb., Fl. Brit. W. Ind., p. 565; Jacq., Ic. rar., vol. 2, pl. 299; Nees in Mart., Fl. Bras., vol. 2, pt. 1, p. 40; Urb., Symb. Ant., vol. 4, p. 112.-El Valle, Miller \& Johnston, no. 182, Aug. 15. Venezuela: Erust, Sobre la flora y fauna, p. 223. Widely distributed in tropical countries.

\section{Dichronient Michx.}

D. ciliata Vahl, Enum., vol. 2, p. 240 (1S06); Urb., Symb. Ant., vol. 4, p. 121. Rhynchospora Vahliana Griseb., Fl. Brit. W. Ind., p. 577 (1S64).- El Valle, Miller \& Johnston, no. 1S9, July 16; San Juan Mt., alt. 500 m., Johnston, no. 201, July 2. Venezuela: Ernst, Sobre la flora y fauna, p. 223. Distribution general from the Southern United States to Brazil. 


\section{Eleocharis R. Br.}

E. Capitata (L.) R. Br., Prod., p. 225 (1S10); Urb., Symb. Ant., vol. 4, p. 117 (Heleocharis). Scirpus capitatus L., Sp. pl., vol. 1, p. 4 S (1753); Griseb., Fl. Brit. W. Ind., p. 570.- El Valle, Miller \& Johnston, no. 191, July 2S, and Johnston, no 201, July 21, alt. 300 m., near small stream on the North Hill. Yenezuela: Ernst in Seem., Journ. bot., 1867 , p. 290-296. Widely distributed in tropical countries.

\section{Finbristylis Tahl.}

F. autumalis (L.) Roem. \& Schult., Syrst., rol. 2, p. 97 (1S17). Scirpus antumnalis L., Mant., vol. 2, p. 180 (1771); Grisel., Fl. Brit. W. Ind., p. 571.- San Juan Mt., alt. 650 m., Johnston, no. 311, Aug. 28. Tenezuela: Ernst, Sobra la flora y fauna, p. 226. Widely distributed from the United States to Brazil.

F. ferruginea (L.) Vahl, Enum., vol. 2, p. 291 (1S06); Urb., Symb. Ant., rol. 4, p. 11S. Scirpus ferruginens L., Sp. pl., ed. 2, rol. 1, p. 74 (1762).- El Valle, Miller \& Johnston, no 1SS, July 2S, and Johnston, no. 205, July 21. Venezuela: Ernst, Sobre la flora y fauna, p. 226. Further distribution, tropical and subtropical countries.

\section{Fuirend Rottb.}

F. umbellata Rottb., Dese. et ic. pl., p. 70, pl. 19, fig. 3 (1773); Griseb., Fl. Brit. W. Incl., p. 573; Nees in Mart., Fl. Bras., rol. 2, pt. 1, p. 107; Urb., Symb. Ant., vol. 4, p. 120.-El Valle, Miller \& Johnston, no. 172, July 24. Venezuela: Ernst in Vargasia, 1S6S-69, p. 1S2. Common in all tropical countries.

\section{Mariscus Gaertn.}

M. ligularis (L.) Urb., Symb. Ant., vol. 2, p. 165 (1900). Cyperns ligularis L., Amoen. acad., vol. 5, p. 391 (1759) et Sp. pl., ed. 2, voll. 1, p. 70.-EI Valle, Miller \& Jolnston, no. 194, July 27. Venezuela: Ernst in Seem., Journ. bot., 1867, p. 290-296. Tropical America and Africa.

\section{Prcreus Beaur.}

P. odoratus (L.) Urb., Symb. Ant., vol. 2, p. $16 \pm$ (1900) et vol. 4, p. 110. Cyperus odoratus L., Sp. pl., rol. 1, p. 46 (175.3), in part.El Valle, Miller \& Johuston, no. 195, Aug. 15. Distribution general in tropical countries. 


\section{Rynchospora Vahl.}

R. barbata (Vahl) Kunth, Enum. pl., vol. 2, p. 290 (1S37); Griseb., Fl. Brit. W. Ind., p. 574. Schoenus barbatus Vahl, Ecl., vol. 2, p. 4 (1798).-San Juan Mt., alt. 600 m., Johnston, no. 207, Aug. 28. Venezuela: Ernst, Sobre la flora y fauna, p. 226. Distribution general in tropical America.

R. Gracilis Vahl, Enum., vol. 2, p. 234 (1806); Griseb., Fl. Brit. W. Ind., p. 574.-San Juan Mt., alt. 600 m., Johnston, no. 310, July 11. Venezuela: Ernst, Sobre la flora y fauna, p. 226.

\section{Hemicarpha Nees \& Arn.}

H. merantha (Vahl) Britton, Bull. Torr. bot. elub, vol. 15, p. 104 (1SS8). Scirpus micranthus Vahl, Enum., vol. 2, p. 254 (1S06).El Valle, Miller \& Johnston, no. 196, Aug. 10. Venezuela: Ernst, Sobre la flora y fauna, p. 226. Distribution general in tropical America.

\section{SCleria Berg.}

S. Bracteata Cav., Ic., vol. 5, p. 34, p]. 457 (1799); Griseb., Fl. Brit. W. Ind., p. 579. Macrolomia bracteata Schrad. ex Nees in Mart., Fl. Bras., vol. 2, pt. 1, p. 182 (1842).- El Valle, Miller \& Johnston, no. 1S7, July 2S; San Juan Mt., alt. 700 m., Johnston, no. 203, July 16. Venezuela: Ernst, Sobre la flora y fauna, p. 226. Distribution general in tropical America.

S. Hirtella Sw., Prod. reg. Ind. Occ., p. 19 (178s); Griseb., Fl. Brit. W. Ind., p. 579.-San Juan MIt., alt. 600 m., Johnston, no. 312, Aug. 28. Venezuela: in woods of Javite, HBK., Nov. gen. et sp., vol. 1, p. 232. Distribution general in tropical America.

S. Lithosperia (L.) Sw., Prod. veg. Ind. Occ., p. 18 (17SS). Scirpus lithospcrmus L., Sp. pl., vol. 1, p. 51 (1753).- San Juan Mt., alt., 600 m., Johnston, no. 32S, Aug. 2S. Venezuela: Ernst, Sobre la flora y fauna, p. 226. Distribution general in tropical countries.

\section{PALMAE.}

\section{Acrocomia Mart.}

A. sclerocarpa Mart., Hist. nat. Palm., vol. 2, p. 66, pls. 56, 57, 100, fig. 5 (1S2:3-50); Griseb., Fl. Brit. WV. Ind., p. 521.- En route El Valle to Juan Griego, alt. $400 \mathrm{~m}$., Johnston, no. 221, July 29. Jamaiea, Grenada, 'Trinidad, Guiana, and Brazil. 


\section{BACTRIS Jacq.}

B. Falcata Johnston, Proc. Amer. acad. arts and sci., vol. 40, p. 683 (1905).- Rio Asuncion, in heavy woods near Juan Griego trail, Johnston, no. 220, July 16.

\section{Cocos L.}

C. Nucifera L., Sp. pl., vol. 2, p. $11 S 8$ (1753); Griseb., Fl. Brit. W. Ind., p. 522; Desc., Ant., vol. 1, pl. 21.- Observed by Loefling, 1754, at Cumaná. Widely distributed in tropical countries. There is a coconut grove extending from El Valle to Porlamar, and another large one in the valley of Asuncion.

\section{Phoenix L.}

P. DACTYlifera L., Sp. pl., vol. 2, p. 1188 (1753); Mart., Hist. nat. Palm. vol. 3, p. 257, pl. 120; Cook, Bull. Torr. bot. club, vol. 2S, p. 528.- Cultivated in EI Valle. Widely grown in tropical countries.

\section{Oreodoxa Willd.}

O. oleracen Mart., Hist. nat. Palm., rol. 3, p. 166, pl. 156, figs. 1, 2 (1836-1850); Griseb., Fl. Brit. W. Ind., p. 517.- San Juan Mt., alt. $600 \mathrm{~m}$., Johnston, no. 222, July 11 . Widely distributed in West Indies.

Palma Carana: "eine kleine Palme mit fächerförmigen Blättern, zur Zeit meines Besuches der Insel, ohne Blüthen und Früchte. Trotz der Aenlichkeit des Namens glaube ich nicht, dass es Mauritia Carana Wall. sein könne" (Ernst). This may well be Acrocomia sclerocarpa above mentioned. An unidentified specimen, no. 347 of Johnston, July 29, alt. $500 \mathrm{~m}$., on the Juan Griego trail is different from any of the above.

\section{ARACEAE.}

\section{Anthurium Schott.}

A. Hookeri Kunth, Enum. pl., vol. 3, p. 74 (1Sł1); Schott, Ic. Aroid., p. 15-17.- San Juan Mt., alt. 400 m., Johnston, no. 297, July 11. Venezuela: Emst, Sobre la flora y fauna, p. 226. Distribution general in the West Indies. 
A. Scandexs (Aubl.) Engl., in Mart., Fl. Bras., rol. 3, pt. 2, p. $7 \mathrm{~S}$ (1578). Dracontium scandens Aubl., Guian., vol. 2, p. 836 (1775).South Hill, El Valle, Johnston, no. 14, July 1S. Found on the branches of trees. Tenezuela: Ernst, Sobre la flora y fauna, p. 226. Brazil.

\section{Dieffenbachia Schott.}

D. segune (Jacq.) Schott, Melet., vol. 1, p. 20 (1832); Griseb., Fl. Brit. W. Ind., p. 509. Arum seguine Jacq., Enum., p. 31 (1760).Rio Asuncion, alt. 400 m., Johuston, no. 214, Aug. 12-15. Venezuela : Ernst, Sobre la flora y fauna, p. 226. Jamaica, French Ids., Guiana.

\section{Philodendron Schott.}

P. eximium Schott, Oestr. bot. Wochenbl., vol. 3, p. 378 (1853).Juan Griego trail, alt. 450 m., Johnston, no. 305, Aug. 12-15. Described from Brazil. Ernst reported an undetermined species of Philodendron from Margarita.

\section{BROMELIACEAE.}

\section{Aechiea R. \& P.}

A. Fendueri André, Bromel. Andreanae, p. 13 (1890); Mez in DC., Monog. Phanerog., vol. 9, p. 223 (1896).- South Hill, El Valle, alt. $335 \mathrm{~m}$. ., Johnston, no. 335; also on the mountain ridge from San Juan Mt. to Juan Griego trail, alt. 500-700 m., Johnston, no. 209, July 2. Venezuela: Ernst, Sobre la flora y fauna, p. 226; between Petaquira and Colonia Tovar, Fendler, no. 245t, according to Mez, l. $c$.

\section{Ananas Adans.}

A. SATryes (Lindl.) Schult. f., Syst., vol. S, p. $12 \$ 3$ (1S30); Rheede, Hort. Mal., vol. 11, pls. 1, 2. Ananassa sativa Lindl. Bot. Reg. vol. 13, under pl. 1068 (1S27); Griseb. Fl. Brit. W. Ind., p. 591.- Cultivated at El Valle, Tacarigua. Distribution general in the West Indies and South America.

\section{BroneliA L.}

B. Pinguin L., Sp. pl., vol. 1, p. 285 (1753); Griseb., Fl. Brit. IT. Ind., p. 591; Red., Lil., vol. 7, p. 396.—El Valle, Miller \& Johnston, no. 247, July 26, and Johnston, no. 211, July 6. Venezuela: Ernst, Sobre la flora y fauna, p. 226. Distribution general from Cuba to Guiana and Colombia. 
Chevalieria Gaudich.

C. SP.- Juan Griego, Emst.

Glomeropitcairnia Mez.

G. ERectiflora Mez, Bull. herb. Boiss., ser. 2, vol. 5, p. 233 (1905). -San Juan Mt., alt. 750 m., Johnston, no. 303. Endemic.

\section{Gravisia Mez.}

G. AQuilega (Salisb.) Mez in Mart., Fl. Bras., vol. 3, pt. 3, p. 300 (1892), nomen, et in DC., Monog. Phanerog., rol. 9, p. 173 (1896). Bromclia aquilega Salisb., Parad., pl. 40 (1805). Aechmea aquilega Griseb., Fl. Brit. W. Ind., p. 592 (1S64).- South Hill, El Valle, alt. 335 m., Johnston, no. 302, July 10. Venezuela: Ernst, Sobre la flora y fauna, p. 226; Angostura, Fcndler, no. 2455. Distribution Tobago, Trinidad, and South America.

\section{Guzinaia R. \& P.}

G. LiNgdlata (L.) Mez in DC., Monog. Phanerog., vol. 9, p. $\$ 99$ (1896). Tillandsia lingulata L., Sp. pl., vol. 1, p. 286 (1753).Juan Griego trail, alt. $100-500$ m., Johnston, no. 301, July 2. Venezuela: Ernst, Sobre la flora y fauna, p. 226. Tropical America.

G. Tricolor R. \& P., Fl. Per., vol. 3, p. 3s, pl. 261 (1802); Griscb., Fl. Brit. IV. Ind., p. 598.- Soutl Hill, El Valle, alt. 300 m., Johnston, no. 295, Aug. 31. Distribution general from the West Indies to Venezuela and Peru.

Thecophyludur André.

T. Johrstonei Mez, Bull. herb. Boiss., ser. 2, vol. 4, p. S72 (1904). - San Juan Mt., alt. 500 m., Johnston, no. 304, July 6. Endemic.

\section{Tillandisia L.}

T. Lescalllei Wright in Samy., Fl. Cub., p. 167 (1S73).-San Juan Mt., alt. 600 m., Johnston, no. 313, July 19. Venezuela: Ernst, Sobre la flora y fauna, p. 226. Cuba.

T. recurvata L., Sp. pl., ed. 2, vol. 1, p. 410 (1762); Griseb., Fl. Brit. W. Ind., p. 598. Renealmia recurvata L., Sp. pl., vol. 1, p. 287 (1753).- Santa Ana, Emst; El Valle, Johnston, no. 219, July 8. 
Venezuela: Cumaná, HBK., Nov."gen. et sp., vol. 1, p. 291. Distribution general in tropical America.

T. utriculata L., Sp. pl., vol. 1, p. 286 (1753).-San Juan Mt., alt. 500 m., Johnston, no. 296, July 27. Venezuela: San Julian, Robinson \& Lyon, July, 1900. The spike is much more crowded than in the typical form. Distribution general in the West Indies and Venezuela.

\section{VRIEsIA Lindl.}

V. Longebracteata Mez in DC., Monog. Phanerog., vol. 9, p. 568 (1896).- Juan Griego trail, alt. 450-550 m., Johnston, no. 300, July 2. Venezuela: Ernst, Sobre la flora y fauna, p. 226; between Petaquira and the sea, Fcndler, no. 2449, acc. to Mez, l.c.

V. SCAlaris Morr., Belg. Hort., vol. 29, p. 301 (1879), also vol. 30, p. 309, pl. 15 (1880).- San Juan Mt., Johnston, no. 294, July 16. Venezuela: Ernst, Sobre la flora y fauna, p. 226. Brazil.

\section{Wittuackia Mez.}

IV. Lingulata (L.) Mez in Mart., Fl. Bras., vol. 3, pt. 3, p. 275 (1892). Bromelia lingulata L., Sp. pl., vol. 1, p. 285 (1753).- South Hill, alt. 300 m., Johnston, no. 294, July 1S. Venezuela: Ernst, Sobre la flora y fauma, p. 226. West Indies.

\section{COMIMELINACEAE.}

\section{Athrrocarpus Schlecht.}

A. persicarifolius (Delile) Hemsl., Biol. Cent.-Am. bot., vol. 3, p. 386 (1SS5). Commelina persicariaefolia Delile in Red., Lil., vol. 8, pl. 472 (1S16).- Rio Asuncion, alt. 400 m., Johnston, no. 309, Aug. 12-15. Venezuela: Ernst, Sobre la flora y fauna, p. 226. Distribution general in tropical America.

\section{Commelina L.}

C. erecta L., Sp. pl., vol. 1, p. 41 (1753); Dill., Elth., vol. 1, pl. 77. - El Valle, Miller \& Johnston, no. 40, July S. Venezuela: Enst, Sobre la flora $y$ fauna, p. 226. Common in the southern U.S.

C. vudiflora L., Sp. pl., vol. 1, p. 41 (1753); Rehb., Hort. bot., vol. 2, pl. 136. C. cayennensis Rich., Act. soc. hist. nat. Par., vol. 1, p. 106 (1792).- Santa Ana, Emst (reported as C. cayennensis Rich.) Tropical countries. 


\section{LILIACEAE.}

\section{ALOË L.}

A. vera L., Sp. pl., vol. 1, p. 320 (1753). A. vulgaris Lam., Encycl., vol. 1, p. 86 (1783); DC., Pl. grasses, pl. 27; Griseb., Fl. Brit. W. Ind., p. 582.- Juan Griego, Ernst. Naturalized and cultivated in the West Indies.

\section{Simlax L.}

S. cumanensis H. \& B. ex Willd., Sp. pl., vol. 4, p. 783 (1S06).San Juan Mt., alt. 600 m., Johnston, no. 134, July 19. Venezuela: near Cumaná and Bordones, HBK., Nov. gen. et sp., vol. 1, p. 272.

\section{AMARYLLIDACEAE.}

\section{Agave L.}

A. americana L., Sp. pl., vol. 1, p. 323 (1753); Griseb., Fl. Brit. IV. Ind., p. 582; Revue horticole, 1862, p. 291.-Santa Ana, Ernst. Distribution general in West Indies and Mexico.

\section{Furcraea Vent.}

F. foetida (L.) Haw., Syn. pl. succ., p. 73 (1S12). Agave foctida L., Sp. pl., vol. 1, p. 323 (1753); Jacq., Ic. pl. rar., vol. 2, pl. 379. F. gigantea Yent., Bull. soc. plilom., vol. 1, p. 65 (1793). Fourcroya gigantea Griseb., Fl. Brit. WV. Ind., p. 582 (1864).- Santa Ana, Ernst. Distribution from the French Ids. to Brazil.

\section{Hruenocallis Salisb.}

H. Caribaea (L.) Herb., Bot. reg., vol. 7, app., p. 44 (1S21). Pancratium caribaeum L., Sp. pl., vol. 1, p. 291 (1753); Griseb., Fl. Brit. IV. Ind., p. 583.- Juan Griego trail, alt. 300 m., Johnston, no. 268, Aug. 14. Tenezuela: Ernst, Sobre la flora y fauna, p. 226. West Indies, Guiana.

\section{Zephyranthes Herb.}

Z. SP.-Bat Cave Peak, El Valle, Johnston, no. 260, July 4. 


\section{DIOSCOREACEAE.}

Dioscorea L.

D. alata L., Sp. pl., vol. 2, p. 1033 (1753); Griseb., Fl. Brit. IV. Ind., p. 587.-Cultivated according to Ernst. Distribution general in the West Indies.

D. Polygonoldes H. \& B. ex Willd., Sp. pl., vol. 4, p. 795 (1S06).Juan Griego trail, alt. 450 m., Johnston, no. 320, Aug. 12-15. Venezuela: between Carichana and Rio Meta, HBK., Nor. gen. et sp., vol. 1, p. 274 .

\section{IRIDACEAE.}

Trimeza Salisb.

T. sP.-San"Juan Mt., alt. 600 m., Johnston, no. 13S, July 11.

\section{MUSACEAE.}

\section{Heliconia L.}

H. Bihai L., Mant., vol. 2, p. 211 (1771); Griggs, Bull. Torr. bot. dub, vol. 30, p. 656. Musa Bihai L., Sp. pl., vol. 2, p. 1043 (1753).- San Juan MIt., alt. $400 \mathrm{~m}$. Venezuela: San Julian, Robinson \& Lyon, July 20, 1900. Common in tropical America.

H PsitTacorum L. f., Suppl., p. 158 (17S1); Griseb., Fl. Brit. WV. Ind., p. 600.- El Valle, Miller \& Johnston, no. 3, July 25; Rio Asuncion, alt. $450 \mathrm{~m}$., Johnston, no. 210, Aug. 12-15. Venezuela: in Valle Caripe, IBKK., Nov. gen. et sp., vol. 1, p. 326. West Indies, Venezuela to Brazil.

\section{Musa L.}

M. paradisiaca L., Sp. pl., vol. 2, p. 1043 (1753), subsp. Normalis (O. Ktze.) Urb., Symb. Aut., vol. 4, p. 156 (1903). M. sapientum. Griseb. Fl. Brit. W. Ind., p. 599. Subsp. SApientum (L.) Urb., Symb. Ant., rol. 4, p. 156 (1903). M. paradisiaca Griseb., Fl. Brit. IV. Ind., p. 599.-Cultivated. Common in tropical countries.

\section{ZINGIBERACEAE.}

\section{Costus L.}

C. Glabratus Sw., Prod. veg. Ind. Occ., p. 11 (17SS); Griseb., Fl. Brit. Wr. Ind., p. 602.- Juan Griego trail, alt. $450 \mathrm{~m}$, Miller \& 
Johnston, no. 213, Aug. 12-15. Venezuela: Ernst, Sobre la flora y fauna, p. 226. Further distribution St. Lucia, Haiti, Guiana.

\section{Renealinia L.}

R. Lutea Johnston, Proc. Amer. acad. arts and sci., vol. 40, p. 683 (1905).- Rio Asuncion, in damp woods along the Juan Griego trail, Johnston, no. 298, Aug. 12-15. Endemic. Plate 26, fig. 1.

\section{MARANTACEAE.}

\section{Calathea G. F. IV. Mey.}

C. Lutea (Aubl.) G. F. IV. Mey., Prim. Fl. Esseq., p. 10 (1818). Maranta lutea Aubl., Guian., vol. 1, p. 4 (1775). C. discolor G. F. W. Mey., 1. c., p. 7 (1818); Griseb., Fl. Brit. W. Ind., p. 604.- Juan Griego trail, alt. $450 \mathrm{~m} .$, Johnston, no. 212, July 29. Venezuela: Ernst, Sobre la flora y fauna, p. 226. Further distribution, Dominica, Trinidad, Guiana, Colombia.

\section{Maranta L.}

M. Arundinacea L., Sp. pl., vol. 1, p. 2 (1753); Griseb., Fl. Brit. IV. Ind., p. 605.- Rio Asuncion, alt. 400 m., Johnston, no. 206 Aug. 12-15. Distribution general in tropical America.

\section{Stromanthe Sond.}

S. толcкат Eichl., Berl. phys. Abh., p. 80 (1SS4). Maranta tonckat Griseb., Fl. Brit. W. Ind., p. 605 (1864).- San Juan Mt., alt. 400 m., Johnston, no. 202, July 16. Venezuela: Ernst, Sobre la flora $\mathrm{y}$ fauna, p. 226. Further distribution, Trinidad, tropical South America.

\section{ORCHIDACEAE.}

\section{Dichaea Lindl.}

D. Gramnomes (Sw.) Lindl., Gen. et sp. Orch., p. 209 (1833). Ep:dendrum graminoides Sw., Prod. veg. Ind. Occ., p. 125 (1788). D. graminea Griseb., Fl. Brit. W. Ind., p. 625 (1864).-San Juan Mt., alt. 600 m., Johnston, no. 242, July 16. Venezuela: Caracas, Lindl., l. c., also Bonpl., vol. 2, p. 15, and Ann. bot., vol. 6, p. \$23. Distribution general in tropical America. 
D. muricata (Sw.) Lindl., Gen. et sp. Orch., p. 209 (1833); Griseb., Fl. Brit. W. Ind., p. 624. Cymbidium muricatum Sw., Act. Ups., vol. 6, p. 71 (1799).-El Valle to Juan Griego, alt. 450 m., Johnston, no. 224, Aug. 12-15. Venezuela: according to Lindl., l.c. Distribution general in the West Indies.

\section{Elleanthus Presl.}

E. Attenuatus Johnston, Proc. Amer. acad. arts and sci., vol. 40, p. 684 (1905).- San Juan Mrt., in wet woods at the summit, alt. 640 m., Miller \& Johnston, no. 270, July 30, and Johnston, no. 233, July 6. Endemic. Plate 26, fig. 2.

\section{EPIDENDRUM L.}

E. Anceps Jacq., Hist. Stirp. Am., p. 224, pl. 138 (1763). E. fuscatum Sm., Spic. bot., p. 21, pl. 23 (1791); Griseb., Fl. Brit. IV. Ind., p. 617.- El Valle, on rocks of a dry river bed on the mountain side, Miller \& Johnston, no. 269, July 29; Juan Griego trail, alt. 150 m., Johnston, no. 232, Aug. 12-15. Venezuela: Ernst, Sobre la flora y fauna, p. 226. Distribution general from Cuba to Guiana.

E. atropurpureun Willd., Sp. pl., vol. 4, p. 115 (1S05).- El Valle, Miller \& Johnston, no. 212, July 5; Bat Cave Peak, Johnston, no. 226, July 4. Found both in flower and in fruit. In 1901, this species was found only on North Hill growing on the tree-like cactus Cereus eburneus. In 1903, there was none in blossom in this place but on the cliffs above the entrance to Bat Cave, alt. about $300 \mathrm{~m}$., the species was growing in abundance. West Indies.

E. cochleatum L., Sp. pl., ed. 2, vol. 2, p. 1351 (1763); Griseb., Fl. Brit. W. Ind., p. 616.- San Juan Mt., alt. 400 m., Johnston, no. 229, July 3. Venezuela: Caracas, see Ann. bot., vol. 6, p. 359. Distribution general from Mexico to Venezuela.

E. Globosum Jacq. Enum. pl. Carib., p. 29 (1760); Griseb., Fl. Brit. W. Ind., p. 619.- San Juan MIt., alt. 400 m., Johnston, no. 230, July 11. Further distribution, Cuba to the French Ids.

E. Johnstoni Ames, Proc. Amer. acad. arts and sci., vol. 40, p. 684 (1905).- San Juan MIt., alt. 600 m., Johnston, no. 236, July 2. Endemic.

E. noctursum Jacq., Enum. pl. Carib., p. 29 (1760); Griseb., Fl. Brit. IT. Ind., p. 619.-San Juan Mt., alt. 650 m., Johnston, no. 
228, July 6. Tenezuela: Caracas, see Ann. bot., vol. 6, p. 404. Distribution general from Cuba to Guiana and Peru.

E. Rigidum Jacq., Enum. pl. Carib., p. 29 (1760); Griseb., Fl. Brit. W. Ind., p. 618.- Juan Griego trail, alt. $450 \mathrm{~m}$., Johnston, no. 231, Aug. 12-15. Very few specimens found. Venezuela: Caracas, see Ann. bot., vol. 4, p. 400 . Distribution general in tropical America.

E. secundum Jacq., Enum. pl. Carib., p. 29 (1760). E. elongatum Jacq., Ic. pl. rar., vol. 3, p. 17, pl. $60+$ (1786-1793); Griseb., Fl. Brit. W. Ind., p. 617.- San Juan Mt., alt. 700 m., Johnston, no. 227, July 6 . Reclining on low shrubs of the mountain top, abundant; flowers pink. Distribution general from the French Ids. to Venezuela.

\section{Hextefia Batem.}

H. sp.-San Juan MIt., alt. 500 m., Johnston, no. 239, July 2. Only three specimens found.

\section{MAXILlaRia R. \& P.}

M. virguxcula Rchb. f., Bonplandia, vol. 2, p. 16 (18.54).- San Juan MIt., alt. 500 m., Johnston, no. 240, July 2. Venezuela: Caracas, see Ann. bot., vol. 6, p. 522, and Xenia Orchid., rol. 1, p. 24, pl. 10.

\section{Orcioica Sw.}

O. Luridum Lindl., Bot. Reg., pl. 727 (1823); Griseb., Fl. Brit. IV. Ind., p. 632.- South Hill, El Valle, Miller \& Johnston, no. 37, July 10; Tacarigua, alt. 300 m., Johnston, no. 306, Aug. 14. Very few flowering specimens. Venezuela: see Ann. bot., vol. 6, p. 7\$1, and Bonpl., vol. 2, p. 13. Widely distributed from Cuba to Venezuela.

\section{Pelexia Poit.}

P. ADnata (Sw.) Spreng., Sỵst., rol. 3, p. 704 (1826); Urb., Șrmb. Ant., vol. 4, p. 16t. Satyrium adnatum Sw., Prod. veg. Ind. Occ., p. 11 (17S8).- El Valle, Miller \& Johnston, no. 116, July 25; San Juan Mt. and Juan Griego trail, alt. 400-500 m., Johnston, no. 243, July 27. Common but rery scattered in the damp woods of the mountain top. Further distribution, West Indies.

Plevrothallis R. Br.

P. Ruscifolia (Jacq.) R. Br. in Ait., Hort. Kew., ed. 2, vol. 5, p. 211 (1S13); Griseb., Fl. Brit. W. Ind., p. 608. Epidendrum rusci- 
folium Jacq., Enum. pl. Carib., p. 29 (1760).- Juan Griego trail, alt. 450 m., Johnston, no. 225, Aug. 12-15. Very abundant, growing in clusters. Venezuela: Caracas, Ernst, Sobre la flora y fauna, p. 268. Distribution general from Cuba to Guiana and Peru.

P. SP. affinis $P$. platycauli Rchb. f.- Juan Griego trail, alt. $600 \mathrm{~m}$., Johnston, no. 241, July 31.

P. Johnstonir Ames, Orchidaceae, vol. 2, p. 271 (1908).-San Juan MIt., alt. 600 m., Johnston, no. 238, July 16 . Endemic.

P. sP. San Juan Mt., Johnston, no. 237, July 16.

P. sp. San Juan Mt., Johnston, no. 235, July 11.

P. sP. San Juan Mt., Johnston, no. 234, July 11.

\section{PIPERACEAE.}

Peperonia R. \& P.

P. glabella (Sw.) A. Dietr., Sp. pl., vol. 1, p. 156 (1831); Griseb., Fl. Brit. W. Ind., p. 165. Piper glabellum Sw., Prod. veg. Ind. Occ., p. 16 (1788).- Bat Cave Peak, El Valle, alt. 300 m., Johnston, no. 12, July 4. Further distribution, Trinidad, Antigua, and Jamaica.

P. scandexs R. \& P., Fl. Per., vol. 1, p. 32, pl. 51, fig. b (1798). P. repens HBK., Nov. gen. et sp., vol. 1, p. 65 (1815); Griseb., Fl. Brit. W. Ind., p. 165.- Santa Ana, Ernst (reported under the name P. repens HBK.); Rio Asuncion, Johnston, no. 13, Aug. 12-15. Abundant on the rocks of the rivulet. Widely distributed in tropical America.

P. victoriana C. DC., var. margaritaxa C. DC., Proc. Amer. acad. arts and sci., vol. 40, p. 685 (1905).- Bat Cave Peak, on the cliffs, alt. 300 m., Johnstou, no. 18, July 4. Endemic.

\section{Piper L.}

P. Johnstoni C. DC., Proc. Amer. acad. arts and sci., vol. 40, p. 685 (1905).- Juan Griego trail, alt. 500 m., Johuston, no. 19, July 22. Endemic.

P. inargaritanum C. DC., Proc. Amer. acad. arts and sci., vol. 40, p. 685 (1905).—El Valle, Miller \& Johnston, no. 216, Aug. 5; San Juan Mt., alt. $700 \mathrm{~m}$., Johnston, no. 20, July 11. Endemic.

P. pseudo-irolliconum C. DC. in DC., Prod., vol. 17, pt. 1, p. 278 (1869).- Juan Griego trail, alt. 450 m., Johnston, no. 17, July 22. Further distribution, Peru. 


\section{ULAIACEAE.}

\section{Celtis L.}

C. Iguanaea (Jacq.) Sarg., Silva, vol. 7, p. 64 (1895); Urb., Symb Ant., vol. 4, p. 194. Rhammus iguanaeus Jacq., Enum., p. 16 (1760). Celtis aculeata Sw., Prod. reg. Ind. Occ., p. 53 (17SS).- Santa Ana, Ernst (reported under the name C. aculeata $\mathrm{Sw}$.); El Valle, Miller \& Johnston, no. 116, Aug. 14, along the trail up the mountain. Venezuela: Colonia Tovar, Fendler, no. 220, 1S54-5j. Further distribution, tropical America. Shrub, 2-3 m. high.

\section{'Trema Lour.}

T. morantha (L.) Blume, Mus. bot. Lugd. Bat., vol. 2, p. 58 (1S5̃3 ?). Rhamnus micranthus L., Syst. nat., ed. 10, vol. 2, p. 937 (1759). Sponia micrantha Dene., Nouv. ann. mus. Par., rol. 3, p. $49 S$ (1S34); Griseb., Fl. Brit. W. Ind., p. 150 (1S64).- Santa Ana, Ernst; San Juan MI., Johnston, no. 245, July 19. Tropical America.

\section{MORACEAE.}

\section{Artocarpus Forst.}

A. Incesa L. f., Suppl., p. 411 (1781); Griseb., Fl. Brit. W. Ind., p. 152; Desc., Ant., vol. \&, pl. 539.-Asuncion, Emst. Further distribution, tropical countries.

\section{Cecropia L.}

C. peltata L., Syrst. nat., ed. 10, vol. 2, p. 1286 (1759); Griseb., Fl. Brit. IV. Ind., p. 153; Desc., Ant., rol. 2, pl. 75.-Santa Ana, Ernst; San Juan Mt., Johnston, no. 132. Common in the woods of the mountain above the Juan Griego trail. Venezuela: Cumaná, HBK., Nov, gen. et sp., vol. 2, p. 43. Further distribution, Jamaica and the Caribbean Ids.

\section{Ficus L.}

F. SP.-- Santa Ana, Ernst.

\section{PROTEACEAE.}

\section{Roupala Aubl.}

R. SP. affinis R. ovali.-Juan Griego trail, alt. $450 \mathrm{~m}$., Johnston no. 330 , Aug. $12-15$. 


\section{OLACACEAE.}

\section{Ninienia L.}

X. anericana L., Sp. pl., vol. 2, p. 1193 (1753); Griseb., Fl. Brit. IV. Ind., p. 310.-El Valle, Johnston, no. 40, Aug. 31. Further distribution, tropical countries.

\section{LORAN'THACEAE.}

\section{LORANTHUS L.}

L. Avicularis Mart. ex Schult. f., Syst., vol. 7, p. 132 (1829); Griseb., Fl. Brit. IV. Ind., p. 312.- Santa Ana, Ernst. Further distribution, tropical America.

L. Emarginatus Sw., Prod. veg. Ind. Occ., p. 5 S (1788); Griseb., Fl. Brit. W. Ind., p. 312.- South Hill, El Valle, alt. 300 m., Johnston, no. 127, July 10. Further distribution, San Domingo.

L. ORInocensis Spreng., Syst., vol. 2, p. 129 (1S25); Griseb., Fl. Brit. W. Ind., p. 311.-El Valle, Miller \& Johnston, no. 250, Aug. 1; North Hill, El Valle, Johnston, no. 264, July 15, parasitic on Bourreria exsucca Jacq.; also common on the trees in the plain between San Antonio and Punta Mosquito. Further distribution, Trinidad and Venezuela.

\section{ARISTOLOCHIACEAE.}

\section{Aristolochia L.}

A. Ringens Vahl, Symb. bot., vol. 3, p. 99 (1794); Griseb., Fl. Brit. IV. Ind., p. 299; Bot. mag., vol. 94, pl. 5700.-Juan Griego trail, alt. 450 m., Johnston, no. 131, July 29. Venezuela: near Buenavista and Los Teques, HBK., Nov. gen. et sp., vol. 2, p. 147; Colonia Tovar, Fendler, no. 1063, 1856-57. Jamaica and Colombia.

\section{POLYGONACEAE.}

\section{Antigonon Endl.}

A. Leptopus Hook. \& Am., Bot. Beech. voy., p. 30s, pl. 69 (1S10); Griseb., Fl. Brit. W. Ind., p. 164.-El Valle, Miller \& Johnston, no. 53, Aug. 1. Venezuela: Sacupana, Rusty \& Squires, no. 35, 
1896. Abundant on fences near dwellings. A garden plant in the West Indies.

\section{Cocoloba L.}

C. Excoriata L., Syst. nat., ed. 10, rol. 2, p. 1007 (1759). C. nivea Jacq., Enum. pl. Carib., p. 19 (1760), and Hist. Stirp. Amer., p. 115, pl. 7S; Griseb., Fl. Brit. WV. Ind., p. 163.-South Hill, El Valle, Johnston, no. 274, Aug. 31. Further distribution, West Indies.

C. Ernsti Johnston, Proc. Amer. acad., arts and sci., vol. 40, p. 685 (1905).- South Hill, El Valle, Johnston, no. 250, Aug. $31 . \quad$ Plate 27, fig. 2

\section{CHENOPODIACEAE.}

\section{Atriplex L.}

A. cristata H. \& B. ex Willd., Sp. pl., vol. 4, p. 959 (1S06). Obione cristata Moq., Chenop. Enum., p. 73 (1S10).- Juan Griego, Ernst; Pt. Moreno, Johnston, no. 316, July 17. Venezuela: at promontory of Araya, HBK., Nov. gen. et sp., vol. 2, p. 192.

\section{Chenopodium L.}

C. Ambrosiomes L., Sp. pl., vol. 1, p. 219 (1753); Griseb., Fl. Brit. W. Ind., p. 60; Fenzl in Mart., Fl. Bras., vol. 5, pt. 1, p. 145; Desc., Ant., vol. 1, pl. 5S.- Santa Ana, Ernst. Venezuela: near Cumaná and Cocollar, HBK., Nov. gen. et sp., vol. 7, p. 191. Further distribution, tropical countries.

\section{SALICORNIA L.}

S. Fruticosa L., Sp. pl., ed 2, vol. 1, p. 5 (1762). S. ambigua Michx., Fl., vol. 1, p. 2 (1S03); Griseb., Fl. Brit. W. Ind., p. 60.Juan Griego, Ernst; Pt. Moreno, Johnston, no. 109, July S. Further distribution, from United States to Peru.

\section{AMARANTHACEAE.}

\section{Achyranthes L.}

A. aspera L., Sp. pl., vol. 1, p. 204 (1753); Griseb., Fl. Brit. IT. Ind., p. 62; Wight, Ic. Ind. Or., vol. 5, p. 1777.-- Juan Griego, Emst. Venezuela: Cumaná, Loefling, Reise nach den spanischen Ländlein, p. 152-153. Further distribution, tropical countries. 


\section{Alternanthera Forsk.}

A. Canescexs HBK., Nor. gen. et sp., vol. 2, p. 204 (1817).- Pt. Moreno, Johnston, no. 317, July S. Venezuela: Cumaná and Bordones, HBK., Nov. gen. et sp., vol. 2, p. 204. Coche, see page 291. Abundant about the lagoon at Pt. Moreno.

A. muscoides (Sw.) Benth. \& Hook. f., Gen., vol. 3, p. 39 (1S80), by implication, though without the exact combination. Lithophila museoides Sw., Prod. veg. Ind. Oce., p. 14 (17SS); Griseb., Fl. Brit IV. Ind., p. 66.- Juan Griego, Ernst. Further distribution, the Lesser Antilles.

\section{Aniranthus L.}

A. Crassipes Schlecht., Linnaea, vol. 6, p. 757 (1831); Urb., Symb. Ant., vol. 4, p. 219.- El Vaile, Miller \& Johnston, no. 29, July 26. Further distribution, tropical America.

A. Paxiculates L., Sp. pl., ed. 2, vol. 2, p. 1406 (1763); Griseb., Fl. Brit. W. Ind., p. 69; Seubert in Mart., Fl. Bras., vol. 5, pt. 1, p. 238; Willd., Hist. Am., pl. 2.- Santa Ana, Ernst. Further distribution, tropical countries.

A. spinosus L., Sp. pl., rol. 2, p. 991 (1753); Griseb., Fl. Brit. II. Ind., p. 68; Seubert in Mart., Fl. Bras., vol. 5, pt. 1, p. 239; Desc., Ant., rol. 5, p. 314.- Santa Ana, Ernst. Further distribution general, especially in tropical countries.

A. Tristis L., Sp. pl., vol. 2, p. 989 (1753); Griseb., Fl. Brit. W. Ind., p. 69; Seubert in Mart., Fl. Bras., vol. 5, pt. 1, p. 237; Wight, Ic. Ind. Or., vol. 2, p. 713.- El Valle, Miller \& Johnston, nos. 149 and 30, Aug. 21. Further distribution, Jamaica to Trinidad, also in Chili.

\section{Crathula Lour.}

C. Prostrata (L.) Bl., Bijlr., p. 549 (1S25); Griseb., Fl. Brit. W. Ind., p. 63; Seubert in Mart., Fl. Bras., vol. 5, pt. 1, p. 230. Achyranthes prostrata L., Sp. pl., ed. 2, vol. 1, p. 296 (1762).- Juan Griego and Santa Ana, Ernst.

\section{Gonphrena L.}

G. PIlosi (Mart. \& Gal.) Moq. in DC., Prod., rol. 13, pt. 2, p. 395 (1849). Mogiphanes pilosa Mart. \& Gal., Bull. acad. Brux., vol. 10, 1).34S (1S43). - El Valle, Miller \& Johnston, no. 260, July 2S; and Johnston, no. 106, July 14. 


\section{IRESINE P. Br.}

I. ELation Rich. ex Willd., Sp. pl., vol. 4, p. 766 (1806); Griseb., Fl. Brit. W. Ind., p. 61; Seubert in Mart., Fl. Bras., vol. 5, pt. 1, p. 226.- - Santa Ana, Ernst.

I. LAtifolia (Mart. \& Gal.) Benth. \& Hook. f., Gen., vol. 3, p. 42 (1880). Gomphrena latifolia Mart. \& Gal., Bull. acad. Brux., vol. 10, p. 349 (1843).-El Valle, Miller \& Johnston, no. 67, July S; Juan Griego trail, Johnston, no. 107, July 2. Shrub, 1-2 m. high; stem often $2.5 \mathrm{~cm}$. in diameter near base.

\section{Philoxerds R. Br.}

P. verimicularis (L.) R. Br., Prod., vol. 1, p. 416 (1810), as vermiculata; Krug, Ic. pl., p. 202; Urb., Symb. Ant., vol. 4, p. 223. Gomphrena vermicularis L., Sp. pl., vol. 1, p. 224 (1753).- Porlamar, Miller \& Johnston, no. 4, July 31, abundant on the low plains near the sea; Pt. Moreno, Johnston, no. 111, July 8.

\section{'Telanthera R. Br.}

T. Ficoined (L.) Moq. in DC., Prod., vol. 13, pt. 2, p. 363 (1St9); Seubert in Mart., Fl. Bras., vol. 5, pt. 1, p. 171. Gomphrena ficoidea L., Sp. pl., yol. 1, p. 225 (1753). Altemanthera ficoidea R. Br., Prod., p. 417 (1810); Griseb., Fl. Brit. IV. Ind., p. 67.- Juan Griego, Emst. Further distribution, tropical America and tropical Africa.

\section{NICTAGINACEAE.}

\section{BOERHatia L.}

B. erecta L., Sp. pl., vol. 1, p. 3 (1753); Griseb., Fl. Brit. W. Ind., p. 69; Schmidt in Mart., Fl. Bras., vol. 14, pt. 2, p. 370; Jacq., Vincl., vol. 1, p. 2, pls. 5, 6; Urb., Symb. Ant., vol. 4, p. 224.-- El Valle, Miller \& Johnston, no. 204, July 20. Further distribution, Georgia, Florida, tropical America to the Galapagos Ids.

B. Paniculata Rich., Act. soc. hist. nat. Par., vol. 1, p. 105 (1792); Griseb., Fl. Brit. IV. Ind., p. 69; Schmidt in Mart., Fl. Bras., vol. 14, pt. 2, p. 369, pl. 86; Urb., Symb. Ant., vol. 4, p. 224.- El Valle, Miller \& Johnston, no. 203, July 10. Tenezuela: Colonia Tovar, Fendler, no. 10\$3, 1854-5.5. Further distribution, tropical America and the Cape Verde Irls. 


\section{Buginvillaea Commerson.}

B. spectabilis Willd., Sp. pl., vol. 2, p. 348 (1799). Bougainvillea spectabilis Schmidt in Mart., Fl. Bras., vol. 14, pt. 2, p. 350 , pl. S2 (1872).- El Valle, Miller \& Johnston, no. 99, July 31, in a yard. Further distribution, tropical America. This specimen is almost as glabrous as B. glabra Choisy.

\section{Mirabilis L.}

M. JALAPA L., Sp. pl., vol. 1, p. 177 (1753); Griseb., Fl. Brit. IV. Ind., p. 69; Schmidt in Mart., Fl. Bras., vol. 14, pt. 2, p. 349.Santa Ana, Ernst. Further distribution, tropical America.

\section{Pisonia L.}

P. aculeata L., Sp. pl., vol. 2, p. 1026 (1753); Griseb., Fl. Brit. W. Ind., p. 70; Schmidt in Mart., Fl. Bras., vol. 14, pt. 2, p. 354.Juan Griego, Ernst. Further distribution, tropics.

P. ineruis Jacq., Select. Am., p. 275 (1763); Griseb., Fl. Brit. IV. Ind., p. 71.- Juan Griego, Ernst; El Valle, Miller \& Johnston, no. 231, July S; Juan Griego trail, alt. 400 m., Johnston, no. 335, Aug. 12-15, also San Juan Mt., alt. 600 in., Johnston, no. 121, Aug. 28. Venezuela: Colonia Tovar, Fendler, no. 1126, June 20, 1855. Further distribution, tropical America.

\section{BATIDACEAE.}

\section{Batis L.}

B. marituia L., Syst. nat., ed. 10, vol. 2, p. 1289 (1759); Griseb., Fl. Brit. IV. Ind., p. 61; Jacq., Hist. stirp. Am., p. 260.-Juan Griego, Ernst; Pt. Moreno, Johnston, no. 108, July 13. Further distribution, from Florida to Venezuela.

\section{PHY'TOLACCACEAE.}

\section{Petiveria L.}

P. Alliaces I., Sp. pl., vol. 1, p. 342 (1753); Griseb., Fl. Brit. IV. Ind., p. 59; Schmidt in Mart., Fl. Bras., vol. 14, pt. 2, p. 332; Urb., Symb. Ant., vol. 4, p. 229.- Santa Ana, Ernst; El Valle, Miller \&. 
Johnston, no. 13, July 15. Venezuela: near Bordones, HBK., Nov. gen. et sp., vol. 2, p. 1S8; Caracas, Birschel; Colonia Tovar, Fendler, nos. 865 and $1805,1854-57$. Further distribution, temperate and tropical America.

\section{Rivina L.}

R. Humilis L., Sp. pl., vol. 1, p. 121 (1753); Schmidt in Mart., Fl. Bras., vol. 14, pt. 2, p. 336; Urb., Symb. Ant., vol. 4, p. $228 . \quad R$. laevis L., Mant., vol. 1, p. 41 (1767); Griseb., Fl. Brit. W. Ind., p. 59.-Santa Ana, Emst; El Valle, Miller \& Johnston, no. 263, July S. Venezuela: Cumaná, Loefling, Reise nach den spanischen Ländern, p. 152-153; Colonia Tovar, Fendler, no. 1088, 1S5+-55; Sacupana, Rusby \& Squires, no. S0, 1S96. Further distribution, tropical America.

\section{AIZOACEAE.}

Crpselea Turp.

C. Humifusa Turp., Ann. mus. Par., vol. 7, p. 219, pl. 121, fig. 5 (1S06); Griseb., Fl. Brit. W. Ind., p. 56.- Shores of Juan Griego pond, Johnston, no. 252, Aug. 14. Further distribution, Caribbean Ids., Haiti to Guadeloupe.

\section{Mollugo L.}

M. verticillata L., Sp. pl., vol. 1, p. $\$ 9$ (1753); Griseb., Fl. Brit. W. Ind., p. 56; Rohrbach in Mart., Fl. Bras., vol. 14, pt. 2, p. 240; Urb., Symb. Ant., vol. 4, p. 230.-Juan Griego, Ernst; El Talle, Miller \& Johnston, no. 21, Aug. 10; Juan Griego, Johnston, no. 126, Aug. 14. This species was seen as a much-branched specimen on the dry roadside and as few-branched specimens on the shores of the Juan Griego pond. Tenezuela: Cumaná, HBK., Nov. gen. et sp., vol. 6, p. 20; Loefling, Reise nach den spanischen Ländern, p. 152-153. Common in tropical America.

\section{Sesuvium L.}

S. portulacastrum L., Syst. nat., ed. 10, vol. 2, p. 1058 (1759); Griseb., Fl. Brit. W. Ind., p. 57; Rohrbach in Mart., Fl. Bras., vol. 14, pt. 2, p. 310.- Juan Griego, Ernst. Venezuela: near Camaná, HBK., Nov. gen. et sp., vol. 6, p. 86; Tortuga and Los Roques, Ernst; near La Guaira, Fendler, no. 62, Aug. 16, 1855. 


\section{Trianthema L.}

'T. portulacastrum L., Sp. pl., vol. 1, p. 223 (1753); Moench, Meth., p. 700; Urb., Symb. Ant., vol. 4, p. 231. T. monogynum L., Mant., vol. 1, p. 69 (1767); Griseb., Fl. Brit. W. Ind., p. 5S.- Juan Griego, Emst; El Valle, Miller \& Johnston, no. 12, July 12; Pt. Moreno, Johnston, no. 110, July S. Very abundant near the lagoon at Pt. Moreno. Tenezuela: La Guaira, Fendler, no. 64, Aug. 1855. Further distribution, tropical America.

\section{POR'TULACACEAE.}

\section{Portulaca L.}

P. Halmoides L., Sp. pl., ed. 2, vol. 1, p. 639 (1762); Griseb., Fl. Brit. W. Ind., p. 57. P. pilosa a Rohrbach in Mart., Fl. Bras., vol. 14, pt. 2, p. 304.- Einst. Found in the West Indies.

P. Milleri Urb., Symb. Ant., vol. 5, p. 344 (1907).- Porlamar, Miller \& Johnston, no. 11 (in part), July 31. Endemic.

P. oleracea L., Sp. pl., vol. 1, p. 445 (1753); Griseb., Fl. Brit. IV. Ind., p. 57.-Common on Margarita, Miller \& Johnston. Tenezuela: Colonia 'Tovar, Fendler, no. 61, 1S5t-55. Widely distributed.

P. pllosa L., Sp. pl., vol. 1, p. 445 (1753); Griseb., Fl. Brit. W. Ind., p. 57; Rohrbach in Mart., Fl. Bras., rol. 14, pt. 2, p. 303.Santa Ana, Ernst.

P. venezuelexsis Lrb., Symb. Ant., vol. 5, p. 314 (1907).Porlamar, Miller \& Johnston, no. 11 (in part), July 31. Found also on the mainland by Gollmer, according to Urban.

\section{Talised Adans.}

T. Paniculatum (Jacq.) Gaertn., Fruct., vol. 2, p. 219, pl. 128 (1791); Urb., Symb. Ant., vol. 4, p. 231. Portulaea paniculata Jacq., Enum., p. 22 (1760). T. patens Willd., Sp. pl., vol. 2, p. $\$ 63$ (1S00); Ernst, Bot. excurs. Margarita, p. 2.- Juan Griego, Ernst; El Valle, Miller \& Johnston, no. 28, Aug. 1. Venezuela: La Guaira, Fendler, no. 66, Aug. 16, 1855. Common in tropical America.

T. triaxglatie (Jacq.) Willd., Sp. pl, vol. 2, p. 862 (1800); Griseb., Fl. Brit. W. Ind., p. 56; Urb., Symb. Ant., vol. 4, p. 231. Portulaea triangularis Jacq., Enum., p. 22 (1760).- T. racemosum Rohrbach in Mart., Fl. Bras., vol. 14, pt. 2, p. 297 (1872).-El Valle, Miller \& Johnston, no. 262, July 20. Common in tropical America. 


\section{MENISPERMACEAE.}

\section{Cissampelos L.}

C. PAReira L., Sp. pl., vol. 2, p. 1031 (1753); Griseb., Fl. Brit. IT. Ind., p. 10; Desc., Ant., rol. 3, p. 231, pl. 201; Urb., Symb. Ant., rol. 4, p. 237.- El Valle, Miller \& Johnston, no. 151, Aug. 2. Venezuela: near Caripe, HBK., Nor. gen. et sp., vol. 5, p. 66; Santa Catalina, Rusby \& Squires, no. 138, 1896. Common to all tropical countries.

\section{ANNONACEAE.}

\section{Annova L.}

A. Reticulata L., Sp. pl., rol. 1, p. 537 (1753); Griseb., Fl. Brit. IV. Ind., p. 5; Mart., Fl. Bras., vol. 13, pt. 1, p. 15; Jacq., Obs., p. 13, pl. 6.- Asuncion, Ernst. Found also in Jamaica, St. Vincent, and Guadeloupe.

A. squanosa L., Sp. pl., vol. 1, p. 537 (1753); Griseb., Fl. Brit. IV. Ind., p. 5; Mart., Fl. Bras., rol. 13, pt. 1, p. 14; Jacq., Hist. Stirp. Am., p. 162; Urb., Symb. Ant., vol. 4, p. 242.- El Valle, Miller \& Johnston, no. 51, July 22. Further distribution, tropical America.

\section{LAURACEAE.}

\section{Nectandra Roland.}

N. Corlacea (Sw.) Griseb., Fl. Brit. IV. Ind., p. 281 (1860); Urb., Symb. Ant., vol. 4, p. 249. Laurus coriacea Sw., Prod. veg. Ind. Occ., p. 65 (1788). N. Willdenoviana Nees, Syst. Laurin., p. 321 (1836).- San Juan Mt., alt. 600 m., Johnston, no. 25S, July 11. Further distribution, West Indies.

N. sp.-San Juan Mt., alt. 600 m., Johnston, no. 336, July 20, and no. 334, Aug. 28. No. 334 has leares smooth and the cupule truncate. It is possibly only $N$. coriacea in fruit.

\section{Phoebe Nees.}

P. cinvaliomifolia (HBK.) Nees, Linnaea, vol. 21, p. 488 (1848). Persea cinnamomifolia HBK., Nov. gen. et sp., vol. 2, p. 160 (1817).San Juan Mt., alt. 600 m., Johnston, no. 267, July 11; also no. 25s, July 11, and no. 336, July 20, at $600 \mathrm{~m}$. alt. Distribution general in American 'Tropics. 


\section{PAPAVERACEAE.}

Argemone L.

A. Mexictina L., Sp. pl., vol. 1, p. 50 S (1753); Lam., Encycl., pl. 452; Griseb., Fl. Brit. W. Ind., p. 13; Eichler in Mart., Fl. Bras., vol. 13, pt. 1, p. 316; Urb., Symb. Ant., vol. 4, p. 250.- Santa Ana, Ernst; El Valle, Miller \& Johnston, no. 245, July 25. Venezuela: near Caracas, Fendler, no. 19, Jan. 14, 1854. Further distribution, Caribbean Ids.

\section{CAPPARIDACEAE.}

\section{Capparis I.}

C. Breynia L., Syst. nat., ed. 10, vol. 2, p. 1071 (1759); Jacq., Hist. Stirp. Am., p. 161, pl. 103; Eichler in Mart., Fl. Bras., vol. 13, pt. 1, p. 271. C. amygdalina Lam., Encycl., vol. 1, p. 608 (1783); Griseb., Fl. Brit. W. Ind., p. 17.- Juan Griego, Emst. Venezuela: Cumaná, HBK., Nov. gen. et sp., vol. 5, p. 97; Loefling, Reise nach den spanischen Ländern, p. 152-153; Valencia, Fendler, no. 2273, Mar. 5, 1857. Further distribution, tropical America.

C. coccolobifolia Mart. ex Eichler in Mart., Fl. Bras., vol. 13, pt. 1, p. 284 (1S65). C. collina Johnston, Proc. Amer. acad. arts and sci., rol. 40, p. 686 (1905).- Tree, $5 \mathrm{~m}$. high, on hillside north of El Valle, Johnston, no. 10, July 15 . Further distribution, tropical America.

C. CrNophallophora L., Sp. pl., ed. 2, vol. 1, p. 721 (1762); Jacq., Hist. Stirp. Am., p. 158; Griseb., Fl. Brit. W. Ind., p. 1S; Eichler in Mart., Fl. Bras., vol. 13, pt. 1, p. 282.-El Valle, Miller \& Johnston, no. 111, July 12; North Hill, El Valle, alt. 200 m., Johnston, no. 5, July 3. A tree, about $5 \mathrm{~m}$. high. Further distribution, tropical America.

C. Jamaicensis Jacq., Enum. pl. Carib., p. 23 (1760), and Hist. Stirp. Am., p. 160; Griseb., Fl. Brit. W. Ind., p. 18; Eichler in Mart., Fl. Bras., vol. 13, pt. 1, p. 270.- El Valle, Miller \& Johnston, no. 106, July 18. Venezuela: Victoria, Fendler, no. 2274, Mar. 4, 1857. Further distribution, West Indies and Venezuela. The Margaritan specimen is very closely allied to $C$. linearis.

C. Linearis Jacq., Enum. pl. Carib., p. 24 (1760), and Hist. Stirp. Am., p. 161.- El Valle, alt. 150 m., Johnston, no. 4, July 3. Tree, about $3 \mathrm{~m}$. ligh; the wood brittle. Venezuela: Golfo de Cariaco, 
Punta Araya, and Laguna Chica, HBK., Nov. gen. et sp., vol. 5, p. 87. Further distribution, northern South America.

C. PACHACA HBK., Nov. gen. et ps., rol. 5, p. 93 (1821); Eichler in Mart., Fl. Bras., vol. 13, pt. 1, p. 281.-- Santa Ana, Ernst; El Valle, Johnston, no. 9, July 3. Venezuela: Cumaná, HBK., Nov. gen. et sp., rol. 5, p. 93 . Tree, about $5 \mathrm{~m}$. in height.

C. Stexosepala Urb., Symb. Ant., vol. 5, p. 529 (1908).-- El Valle, alt. $200 \mathrm{~m}$., Johnston, no. 8, July 27 . Tree, about $7 \mathrm{~m}$. high; flowers yellowish outside, purple within. Endemic. Occurs in Coche.

C. tenuisiliqua Jacq., Enum. pl. Carib., p. 24 (1760); Eichler in Mart., Fl. Bras., vol. 13, pt. 1, p. 27S.-El Valle, Miller \& Johnston, no. 9, July 13; also Johnston, no. 6, July 21, and in fruit Aug. S, alt. 150 m. Venezuela: Colonia Tovar, Fendler, no. 30, Aug. 16. 1855. Further distribution, Colombia.

C. verrucosa Jacq., Enum. pl. Carib., p. 23 (1760), and Hist. Stirp. Am., p. 159; Griseb., Fl. Brit. W. Ind., p. 19.-- North and South Hills, alt. 60-300 m., El Valle, Johnston, no. 1, July 26. Shrub, 2-3 m. high. Venezuela: Cumaná, HBK., Nov. gen. et sp., vol. 5, p. 90; Colonia Tovar, Fendler, no. 34 B, Aug. 16, 1855. Further distribution, tropical America.

\section{Crateva L.}

C. Grivandra L., Sp. pl., ed. 2, vol. 1, p. 636 (1762); Griseb., Fl. Brit. IV. Ind., p. 17.- Santa Ana, Ernst; El Valle, Johnston, no. 2, July 1. Venezuela: Cumaná, HBK., Nov. gen. et sp., vol. 5, p. S6; Turmera, Fendler, no. 1893, Mar. 14, 1857. Common in tropical America. Tree, about $20 \mathrm{~m}$. high, with a trunk $7 \mathrm{dm}$. in diameter at base.

\section{GrNandropsis DC.}

G. Pentaphylla DC., Prod., vol. 1, p. 238 (1S24); Eichler in Mart., Fl. Bras., vol. 13, pt. 1, p. 261, pl. 58, fig. 3; Urb., Symb. Ant., vol. 4, p. 252.-El Valle, Miller \& Johnston, no. 73, July 29. Distribution general in tropical countries.

\section{Morisonia L.}

M. americaxa L., Sp. pl., vol. 1, p. 503 (1753); Griseb., Fl. Brit. IV. Ind., p. 19.-- Santa Ana, Ernst. Tenezuela: Colonia Tovar, Fendler, no. 2472, Jan. 6, 1855. West Indies. 
M. Johnstonir Urb., Simb. Ant., vol. 5, p. 348 (1907).- El Valle, alt. $300 \mathrm{~m}$., Johnston, no. 7, July 10. A tree, about $7 \mathrm{~m}$. high; trunk $6 \mathrm{dm}$. in diameter at base; wood of strong odor, brittle. Endemic.

\section{Steriphoila Spreng.}

S. Elliptica Spreng., Syst., vol. 4, pt. 2, p. 139 (1827); Griseb., Fl. Brit. IV. Ind., p. 20.-El Valle, Miller \& Johnston, no. 267, July 30, and Johnston, no. 3, July 15, alt. 60-300 m. Small tree, $5 \mathrm{~m}$. high. Further distribution, Trinidad and Cumaná.

\section{CRUCIFERAE.}

\section{Cakile Juss.}

C. Aequalis L'Hér. ex DC., Syst., vol. 2, p. 430 (1S21); Griseb., Fl. Brit. W. Ind., p. 14; Deless., Ic., vol. 2, p. 17, pl. 57.-Juan Griego, Ernst. Tenezuela: Ernst, Sobre la flora y fauna, p. 223. Further distribution, West Indies.

\section{LEPIDIUII L.}

L. virginicuni L., Sp. pl., vol. 2, p. 645 (1753); Griseb., Fl. Brit. W. Ind., p. 14.- Santa Ana, Ernst. Common to the West Indies and eastern Lnited States.

\section{LEGUMINOSAE.}

\section{Abrus L.}

A. precatorius L., Syst. nat., ed. 12, vol. 2, p. 472 (1767); Griseb., Fl. Brit. W. Ind., p. 190; Desc., Ant., vol. 4, p. 194, pl. 275.- Juan Griego and Santa Ana, Ernst. Common in tropics.

\section{ACACIA Mill.}

A. Macracantha H. \& B. ex Willd., Sp. pl., vol. 4, p. 1080 (1806); Griseb., Fl. Brit. WT. Ind., p. 221; Kunth, Mim., p. 90, pl. 28.-El Valle, Miller \& Johnston, no. 114, Ang. 2, and River trail, El Valle, Johnston, no. 28, July 15. Venezuela: Colonia Tovar, Fendler, no. 362, 1S54-55. Slender shrub, $5 \mathrm{~m}$. high; flowers yellow. Further distribution, tropical America.

A. Panicclata Willd., Sp. pl., vol. 4, p. 107t (1S06); Griseb., 
Fl. Brit. W. Ind., p. 221; Mart., Fl. Bras., vol. 15, pt. 2, pl. 102.Santa Ana, Ernst. Venezuela: Colonia Tovar, Fendler, no. 351, 1S51-55. Further distribution, St. Lucia, Cuba, Guiana, and Brazil.

A. Tamarindifolia (L.) Willd., Sp. pl., vol. 4, p. 1092 (1806); Griseb., Fl. Brit. W. Ind., p. 221. Mimosa tamarindifolia L., Sp. pl., vol. 1, p. 523 (1753).-El Yalle, Miller \& Johnston, no. 113, July 15; en route Peilar to Pt. Moreno, Johnston, no. 37, July S. Venezuela: Bordones, HBK., Nor. gen. et sp., rol. 6, p. 277; La Guaira, Robinson \& Lyon, July 12, 1900. Slender shrub, 5 m. high. Tropical America.

\section{Bachixia L.}

B. cumanevis HBK., Nov. gen. et sp., vol. 6, p. 321 (1823); Bot. reg., vol. 14, pl. 1133. - South Hill, El Valle, Miller \& Jolnnston, no. 70, July 27. Observed on North Hill, Aug. 15, 1903, not in flower. Venezuela: Bordones, HBK., Nov. gen. et sp., rol. 6, p. 277. Further distribution, northern South America.

\section{Caesalpinia L.}

C. Corlaria (Jacq.) Willd., Sp. pl., vol. 2, p. 532 (1799). Libidibia coriaria Schlecht., Linnaea, vol. 5, p. 193 (1830); Griseb., Fl. Brit. IT. Ind., p. 206. Poinciana coriaria Jacq., Hist. Stirp. Amı, p. 123, pl. 175 (1763).- Santa Ana, Emst; El Valle, Johnston, no. 29. Further distribution, tropical America.

C. Pulcherrina (L.) Sw., Obs., p. 166 (1791); Griseb., Fl. Brit. IV. Ind., p. 205.- Santa Ana, Ernst; EI Valle, Miller \& Johnston, no. 64, July 22. Common in tropical countries.

\section{Cajanus DC.}

C. Ixdicus Spreng., Srst., vol. 3, p. 248 (1S26); Griseb., Fl. Brit. IV. Ind., p. 191; Urb., Ș̣mb. Ant., rol. 4, p. 306. Poinciana puleherrima L., Sp. pl., vol. 1, p. 380 (1753). - El Valle, Miller \& Johnston, no. 139, Aug. 6. Venezuela: Caracas, Birschel; Santa Catalina, Rusby \& Squires, no. 191, 1896; Colonia 'Tovar, Fendler, no. 303, 1S54-5.5. Common in tropical countries.

\section{Calliandra Benth.}

C. portoricensis Benth. in Hook., Lond. journ. bot., rol. 3, p. 99 (1844); Griseb., Fl. Brit. W. Ind., p. 224. Mimosa portoricensis 
Jacq., Ic. pl. rar., vol. 3, p. 20, pl. 633 (1793)._-El Valle, Miller \& Jolunston, no. 264, July 29. Tropical America.

C. Panlosia Johnston, Proc. Amer. acad. arts and sci., vol. 40, p. 686 (1905).- Abundant on hills at alt. 300-600 m., El Valle to Juan Griego, Miller \& Johnston, no. 58, July 22, and Johnston, no. 27, July 2. Endemic.

\section{Canavaluia DC.}

C. obtusifolia (Lam.) DC., Prod., rol. 2, p. 404 (1825); Griseb., Fl. Brit. W. Ind., p. 197; Rlieede, Hort. Mal., vol. 8, pl. 43. Dolichos obtusifolius Lam., Encycl, vol. 2, p. 295 (1786).- Juan Griego, Emst. Common in tropical countries.

\section{Cassia L.}

C. BIflora L., Sp. pl., vol. 1, p. 378 (1753); Griseb., Fl. Brit. W. Ind., p. 208; Bot. reg., vol. 16, pl. 1310.--Santa Ana, Emst; El Valle, Miller \& Johnston, no. 61, July 22. Further distribution, tropical America.

C. emarginata L., Sp. pl., vol. 1, p. 376 (1753); Griseb., Fl. Brit. IV. Ind., p. 207; Urb., Symb. Ant., vol. 4, p. 27t.-El Valle, Miller \& Johnston, no. 201, July 6, and also Johnston, no. 24, July 1.5. Growing in the River trail in El Valle and also on the hillside. Venezuela: Cumaná, Loefling, Reise nach den spanischen Ländern, p. 152153. Found also in Jamaica, St. Vincent, and Haiti.

C. Fistula L., Sp. pl., rol. 1, p. 377 (1753); Griseb., Fl. Brit. W. Ind., p. 206; Desc., Ant., vol. 2, p. 231, pl. 125; Urb., Symb. Ant., rol. 4, p. 272.- El Valle, Miller \& Johnston, no. 69, July 15. Venezucla: Ernst, Sobre la flora $y$ fauna, p. 226. Further distribution, tropical countries.

C. Nictitans L., Sp. pl., vol. 1, p. 380 (1753); Griseb., Fl. Brit. IV. Ind., p. 211; Urb., Symb. Ant., vol. 4, p. 276.- Margarita, according to Urb., l. c., p. 276; Santa Ana, Ernst. Distribution general in American 'Tropics and Sub-tropics.

C. obovata Collad., Hist. Cas., p. 92, pl. 15 (1816); Griseb., Fl. Brit. W. Ind., p. 209.-- El Valle, Miller \& Johnston, no. 228, July 26. Also in Jamaica, according to Griseb., introduced from tropical Africa.

C. occinentalis L., Sp. pl., vol. 1, p. 377 (1753); Griseb., Fl. Brit. W. Ind., p. 209; Desc., Ant., vol. 2, p. 277, pl. 135; Urb., Symb. Ant., vol. 4, p. 273.- Santa Ana and Asuncion, Ernst; El Valle, Miller 
\& Johnston, no. 60, July 11. Tenezuela: Cumaná, Loefling, Reise nach den spanischen Ländern, p. 152-153. Common in tropical countries.

C. oxyphylla Kunth, Mimos., p. 129, pl. 39 (1819-24).-El Valle, Miller \& Johnston, no. 62, Aug. 5. Venezuela: on Mt. Cocol- . lar, HBK., Nov. gen. et sp., vol. 6, p. 342; La Guaira, Robinson \& Lyon, July 6, 1900. Further distribution, Mexico to Ecuador.

C. SERicea Sw., Prod. reg. Ind. Occ., p. 66 (17SS), and Fl. Ind. Occ., rol. 2, p. 72t; Griseb., Fl. Brit. W. Ind., p. 209; Mart., Fl. Bras., vol. 15, pt. 2, p. 116, pl. 35.- El Valle, Miller \& Johnston, no. 56, July 5. Further distribution, Cuba to Panama and Brazil.

C. stenocarpa Tog., Syn. Cass., p. 68 (1837).-El Valle, Miller \& Johnston, no. 210, July 14. Venezuela: Colonia Tovar, Fendler, no. 321, 185t-55. Very closely allied to C. nictitans L., if not identical with it. Found also in Brazil.

C. Tora L., Sp. pl., vol. 1, p. 376 (1753). C. obtusifolia L., Sp. pl., vol. 1, p. 377 (1753); Griseb., Fl. Brit. W. Ind., p. 209.-Santa Ana, Ernst. Further distribution, tropical countries.

\section{Centrosena Benth.}

C. Brasilianua Benth., Ann. Wien. Mus., rol. 2, p. 115 (1837); Mart., Fl. Bras., vol. 15, pt. 1, p. 12S, pl. 34. Bradburya brasiliana Ktze., Rev. gen., vol. 1, p. $16+$ (1S91).-Asuncion, Miller \& Johnston, no. 54, Aug. 9. Venezuela: Colonia 'Tovar, Fendler, no. 1778, 1S5657. Found also in Brazil.

C. virginlanum (L.) Bentl., Ann. Wien. Mus., vol. 2, p. 120 (1S37), Urb., Symb. Ant., vol. 4, p. 200. Clitoria virginiana L., Sp. pl., vol. 2, p. 753 (1753); Bot. reg., vol. 13, p. 1047. Bradburya virginiana Ktze., Rer. gen., vol. 1, p. $16 \pm$ (1S91)._El Valle, Miller \& Johnston, no. 256, Aug. 15. Further distribution, tropical America and Nigeria.

\section{Cercidium Tulasne.}

C. spinosum 'Tulasne, Arch. mus. Par., vol. 4, p. 134 (18t4).-- El Valle, alt. 200 m., Johnston, no. 31, July 3. Shrub, 3 m. high; trunk $2 \mathrm{dm}$. in diameter at base; bark green; stem thorny; flowers vellow. Further distribution, Brazil.

C. viride (Karst.) Taub. in Engl. \& Prantl, Natürl. Pflanzenfam., vol. 3, pt. 3, p. 172 (1892). Rhetimophloeum viride Karst., Fl. Columb., vol. 2, p. 25, pl. 113 (1S62).- Santa Ana, Ernst. Found in northern South America. 


\section{Cititoria I.}

C. Ternatea L., Sp. pl., vol. 2, p. 753 (1753); Griseb., Fl. Brit. IV. Ind., p. 192; Garden, vol. 3S, p. 132, pl. 765; Urb., Symb. Ant., vol. 4, p. 299.-El Valle, Miller \& Johriston, no. 55, July 15, and Johnston, no. 36, July 15. 'Tropical countries.

\section{Cracca Benth.}

C. CARIBaEA Benth. ex Oerst. in Kjoeb. Vidensk. meddel., p. 9, (1S53); Griseb., Fl. Brit. W. Ind., p. 1S3.- El Valle, Miller \& Johnston, nos. 135 \& 141, July 27 and Aug. 2, respectively. Further distribution, tropical America.

\section{Crotalaria L.}

C. Ixcana L., Sp. pl., vol. 2, p. 716 (1753); Griseb., Fl. Brit. IV. Ind., p. 1S0; Cav., Ic., pl. 322; Urb., Symb. Ant., vol. 4, p. 2S1.Santa Ana, Ermst; El Valle, Miller \& Johnston, no. 50, July S. Further distribution, tropical America and tropical Africa.

\section{Desmanthus Willd.}

D. virgatus IVilld., Sp. pl., vol. 4, p. $10+7$ (1S06); Griseb., Fl. Brit. IV. Ind., p. 21S.- Santa Ana, Ernst. Further distribution, temperate and tropical America and East Indies.

\section{Desilodical Desv.}

D. Scorpitrus (Sw.) Desv., Journ. bot., vol. 1, p. 122 (1813); Griseb., Fl. Brit. IV. Ind., p. 187; [rb., Symb. Ant., vol. 4, p. 293. Hedysarum Scorpiurns Sw., Prod. veg. Ind. Oce., p. 107 (17SS).El Valle, Miller \& Johnston, no. 214, July 12. Further distribution, tropical America and Society Ids.

D. spirale (Sw.) DC., Prod., vol. 2, p. 332 (1825); Griseb., Fl. Brit. W. Ind., p. 18S. Hedysarum spirale Sw., Prod. veg. Ind. Occ., p. 107 (17Ss). - Santa Ana, Ernst. Further distribution, tropical countries.

D. supinua (Sw.) DC., Prod., vol. 2, p. 332 (1S25); Urb., Symb. Ant., vol. 4, p. 290. Hedysarum supimm Sw., Prod. veg. Ind. Occ., p. 106 (178S). D. incanum DC., Prod., vol. 2, p. 332 (1825).- El Valle, Miller \&. Johnston, no. 123, July 1t. Further distribution, tropical countries. 


\section{Dolichos L.}

D. SP.- Ernst.

Enterolobium Mart.

E. cyclocarpuy (Jacq.) Griseb., Fl. Brit. W. Ind., p. 226 (1560). Mimosa cyclocarpa Jacq., Frag., p. 30, pl. 34, fig. 1 (1809).- Santa Ana, Ernst. Occurs also in Jamaica and Venezuela.

\section{Erioseira Desv.}

E. CRinitum (HBK.) G. Don, Syst., rol. 2, p. 345 (1832). Glycine crinita HBK., Nov. gen. et sp., vol. 6, p. 421 (1S23).- Santa Ana, Emst. Further distribution, tropical America.

\section{ERTTHRINA L.}

E. Corallodexdrum L., Sp. pl., rol. 2, p. 706 (1753); Griseb., Fl. Brit. W. Ind., p. 199.- El Valle, Johnston, no. 259, July 16. Venezuela, according to Ernst in World's Columbian exposition at Chicago. Further distribution, from Mexico to Brazil.

\section{GLIRICIDIA HBK.}

G. Lutex Johnston, Proc. Amer. acad. arts and sci., vol. 40, p. 687 (1905).- On dry hillside, El Valle, Miller \& Johnston, no. 246, and Johnston, no. 34, Aug. 15. Endemic. Plate 30, fig. 1, 1a-d.

\section{Hyaienea L.}

H. Courbaril L., Sp. pl., vol. 2, p. 1192 (1753); Griseb., Fl. Brit. IV. Ind., p. 213.- El Valle, Johnston, no. 290, Aug. Venezuela: near Carichana, HBK., Nor. gen. et sp., rol. 6, p. 32.2. Further distribution, Cuba, Panama, Guiana.

\section{INDIGOFERA L.}

I. subulata Vahl ex Poir., Suppl., vol. 3, p. 150 (1S13); Griseb., Fl. Brit. W. Ind., p. 181. - El Valle, Willer \& Johnston, no. 142, July 26. Further distribution, Mexico to Venezuela and in East Indies.

I. suffruticosa Mill., Gard. dict., ed. S, no. 2 (1768). I. Anil L., Mant., vol. 2, p. 272 (1771); Urb., Symb. Ant., vol. 4, p. 1S1.-- Santa Ana, Ernst; El Valle, Jiller \& Johnston, no. 52, July 26, and Johnston, no. 35, July 24. Further distribution, temperate and tropical countries. 


\section{INGA Scop.}

I. ingomes (Rich.) Willd., Sp. pl., vol. 4, p. 1012 (1806); Griseb., Fl. Brit. W. Ind., p. 228. Mimosa ingoides Rich., Act. soc. hist. nat. Par., vol. 1, p. 113 (1792).- Juan Griego trail, alt. 400 m., Johnston, no. 26, Aug. 12-15. Tree, about $20 \mathrm{~m}$. high. Further distribution, West Indies and Guiana.

I. macrantha Johnston, Proc. Amer. acad. arts and sci., vol. 40, p. 687 (1905).- On Juan Griego trail, alt. 400 m., Johnston, no. 25, July 11. Endemic. Plate 28, fig. 1.

\section{LONCHOCARPUS HBK.}

L. Latifolius (Willd.) HBK., Nor. gen. et sp., vol. 6, p. 383 (1823); Griseb., Fl. Brit. IV. Ind., p. 199. Amerimmum latifolium Willd., Sp. pl., vol. 3, p. 909 (1S03).-Santa Ana, Ernst. Further distribution, tropical America.

L. velutinus Benth. in Seem., Bot. voy. Herald., p. 111 (1853).El Valle, Johnston, no. 23, July 7. Further distribution, Central America.

L. violaceus (Jacq.) HBK., Nov. gen. et sp., vol. 6, p. 383 (1823), in note; Griseb., Fl. Brit. W. Ind., p. 200. Robinia violacea Jacq., Enum. pl. Carib., p. 28 (1760). - El Valle, Miller \& Johnston, no. 257, July 21; and Jolenston, no. 22, Aug. Further distribution, tropical America. Trec, about $15 \mathrm{~m}$. high.

\section{Machaerium Pers.}

M. striatum Johnston, Proc. Amer. acad. arts and sci., vol. 40, p. 688 (1905). - North Hill, El Talle, Johnston, no. 124. Aug. S. Enmemic. Plate 28, fig. 2.

\section{Mrmosa L.}

"M. FAstiglata IV." acc. to Emst (a combination wholly obscure and probably due to some clerical error).- Santa Ana, Ernst.

\section{Myrosperium Jacq.}

M. Frutescens Jacq., Enum. pl. Carib., p. 20 (1760); Griseb., Fl. Brit. W. Ind., p. 204.- Juan Griego, Ernst. Venezuela: near Parapara and on the banks of the Rio Guarico, HBK., Nov. gen. et sp., vol. 6, p. 372. Further distribution, Trinidad and Colombia. 
Nissolia Jacq.

N. Wislizeni Gray, Journ. Linn. soc., vol. 5, p. 25 (1S61). Chaetocalyx Wistizeni Gray, Pl. Wright., vol. 1, p. 51 (1S52).— El Valle, Miller \& Johnston, no. 259, Aug. 4. Further distribution, Mexico.

\section{Parkinsonia L.}

P. aculeata L., Sp. pl., p. 375 (1753); Griseb., Fl. Brit. WV. Ind., p. 204; Urb., Symb. Ant., vol. 4, p. 277.-Asuncion, Ernst; El Valle, Miller \& Jolunston, no. 63, July 20. Venezuela: Cumaná, Loefling, Reise nach den spanischen Ländern, p. 152-153. Found in all tropical countries.

\section{Peltophorum Walp.}

P. acutifolium, n. comb. Caesalpinia acutifolia Johnston, Proc. Amer. acad. arts and sci., vol. 40, p. 686 (1905). Peltophorum Suringari Urb., Symb. Ant., vol. 5, p. 363 (1908).- On the hillside, El Valle, Miller \& Johnston, no. 200, Aug. 15, and Johnston, no. 33, Aug. 3. Found also on Aruba and Curaçoa.

\section{Phaseolus L.}

P. Lunatus L., Sp. pl., vol. 2, p. 724 (1753); DC., Prod., vol. 2, p. 393; Benth. in Mart., Fl. Bras., vol. 15, pt. 1, p. 181; Urb., Symb. Ant., vol. 4, p. 308.- Margarita according to Urban, l. c. Distribution general in tropical and temperate countries; cultivated.

P. SP.-Ernst.

P. sP.-El Valle, Miller \& Johnston, no. 57, July 26. Possibly this is a specimen of the above $P$. lunatus.

\section{Pithecolobium Mart.}

P. Ligustrinum (Jacq.) Klotzsch ex Benth., Trans. Linn. soc., vol. 30, p. 571 (1875). Mimosa ligustrina Jacq., Fragm., p. 29, pl. 32, fig. 6, not Vahl.-El Valle, Willer \& Johnston, no. 242, July 30, and no. 121; Johnston, no. 38, July 1. Tree, about $8 \mathrm{~m}$. high, and $1.2 \mathrm{dm}$. in diameter at its base. Further distribution, American tropics.

P. unguis-Cati (L.) Benth. in Hook., Lond. journ. bot., vol. 3, p. 200 (1S44); Griseb., Fl. Brit. W. Ind., p. 226. Mimosa unguis cati L., Sp. pl., vol. 1, p. 517 (1753).—Santa Ana, Emst; El Valle, 
Johnston, no. 43, July 14. Tenezuela: Cumaná, Loefling, Reise nach den spanischen Ländern, p. 152-153. A slender tree, about $5 \mathrm{~m}$. high, branching copiously and growing in clumps. Common in tropical America.

\section{Platyuiscium Vog.}

P. polystachycu Benth. in Seem., Bot. voy. Herald., p. 111, pl. 21 (1553). P. platystachyum Griseb., Fl. Brit. W. Ind., p. 200 (1860). - Santa Ana, Ermst. Further distribution, St. Vincent, Trinidad, Panama, and Venezuela.

\section{Polnciana L.}

P. Regia Boj. ex Hook., Bot. mag., pl. 28\$4 (1829); Urb., Symb. Ant., vol. 4, p. 278.- El Valle, Miller \& Johnston, no. 59, July 29. Common in tropical countries.

\section{Prosopis L.}

P. juliflora (Sw.) DC., Prod., vol. 2, p. 447 (1S25); Griseb., Fl. Brit. W. Ind., p. 217. Mimosa juliflora Sw., Prod. veg. Ind. Occ., p. 8.5 (17S8). P. cumanensis HBK., Nov. gen. et sp., vol. 6, p. 310 (1823).- Santa Ana, Ernst; El Valle, Johnston, no. 42, July 15. A tree, about $5 \mathrm{~m}$. high, with wide-spreading top. Further distribution, Mexico to Guayaquil, also Jamaica.

\section{Rhynchosia Lour.}

R. minma (L.) DC., Prod., vol. 2, p. 385 (1825); Griseb., Fl. Brit. W. Ind., p. 190; Urb., Symb. Ant., vol. 4, p. 307. Dolichos minimus L., Sp. pl., vol. 2, p. 726 (1753).-El Valle, Miller \& Johnston, no. 118, July 19. Common in tropical countries.

\section{Strlosanthes Sw.}

S. numls HBK., Nov. gen, et sp., vol. 6, p. 506, pl. 594 (1S23).Asmucion, Miller \& Johnston, no. 68, Aug. 9. Venezuela: near Carichana, HBK., l. $c$.

S. viscosa Sw., Prod. reg. Ind. Occ., p. 108 (17S8); Griseb., Fl. Brit. W. Ind., p. 1Ss.- El Talle, Miller \& Johnston, no. 268, July 30; Pt. Mosquito, Jolnston, no. 249, Aug. 10. Further distribution, tropical America.

\section{Tamarindus $\mathrm{L}$.}

T. INDica L., Sp. pl., vol. 1, p. 31 (1753); Griseb., Fl. Brit. W. Ind., p. 213; Urb., Symb. Ant., vol. 4, p. 270.- EI Valle, Willer \& Johnston, 
no. 65, July 21. Venezuela: Cumaná, Loefling, Reise nach den spanischen Ländern, p. 152-153. Common to all tropical countries.

\section{Tephrosia Pers.}

T. cinerea (L.) Pers., Syn., vol. 2, p. 328 (1S07); Griseb., Fl. Brit. W. Ind., p. 182; Urb., Symb. Ant., vol. 4, p. 283. Galega cinerea L., Syst. nat., ed. 10, rol. 2, p. 1172 (1759).- Santa Ana, Juan Griego, Ernst; El Valle, Miller \& Johnston, no. 136, July 20. Further distribution, tropical America.

\section{Teramus Sw.}

T. uncinates (L.) Sw., Prod. veg. Ind. Occ., p. 105 (17SS); Griseb., Fl. Brit. W. Ind., p. 193. Dolichos uncinatus L., Sp. pl., ed. 2, vol. 2, p. 1019 (1763).- Juan Griego, Santa Ana, Emst. Further distribution, tropical America.

\section{Vigna Savi.}

V. vexillata (L.) A. Rich. in Sagra, Cub., vol. 10, p. 191 (1S45); Griseb., Fl. Brit. W. Ind., p. 195. Phaseolus vexillatus L., Sp. pl., vol. 2, p. 724 (1753).- Santa Ana, Emst. Distributed generally in tropical countries.

\section{Zoria Gmel.}

Z. Diphylla (L.) Pers., Syn., vol. 2, p. 318 (1807); Griseb., Fl. Brit. IV. Ind., p. 1S5. Hedysarum diphyllum L., Sp. pl., vol. 2, p. 747 (1753).- Santa Ana, Emst. Further distribution, general in tropical countries.

\section{OXALIDACEAE.}

\section{Oxalis L.}

O. Barrelieri Jacq., Oxal., p. 24, pl. 3 (1794); Griseb., Fl. Brit. Wr. Ind., p. 133.- El Valle, Miller \& Johnston, no. 122, July 30. Further distribution, tropical America.

O. Plumeri Jacq., Oxal., p. 23 (1794). O. frutescens Griseb., Fl. Brit. IV. Ind., p. 133 (1S59), not L.-San Juan Mt., alt. 795 m., Johnston, no. 128, July 6. Further distribution, Dominica, St. Vincent, and Martinique. 


\section{ER I'THROXYLACEAE.}

\section{Erythroxylum P. Br.}

E. havanense Jacq., Stirp. Am., p. 135, pl. 87, fig. 2 (1763). $E$. ovatum Cav., Diss., p. 40t, pl. 233 (1789); Griseb., Fl. Brit. W. Ind., p. 113.- Santa Ana, Emst; El Valle, River trail, Johnston, no. 72, Aug. 20. Slender tree, $5 \mathrm{~m}$. high; flowers small, white. Further distribution, Dominica, Trinidad, Guiana, and Bahia.

\section{Stigmatophyllui Juss.}

S. SP.- Santa Ana, Ernst.

\section{ZYGOPHYLLACEAE.}

\section{Guajacum L.}

G. Arboreum (Jacq.) DC., Prod., vol. 1, p. 707 (1824). Zygophyllum arboreum Jac(.., Stirp. Am., p. 130, pl. \$3 (1763). - Santa Ana, Ernst; El Valle, Miller \& Johnston, no. 20, July 6; Johnston, no. 30, Aug. 8. Common on the road between Porlamar and Juan Griego by way of Asuncion. Further distribution, tropical America.

G. officinale L., Sp. pl., vol. 1, p. 381 (1753); Griseb., Fl. Brit. IV. Ind, p. 134.- Pt. Mosquito, Johnston, no. 117, Aug. 10. Venezuela: Ernst, Sobre la flora y fauna, p. 226. Distribution general from Cuba to Venezuela.

\section{Kallstroemia Scop.}

K. maxima (L.) Wight \& Arn., Prod., vol. 1, p. 145 (1834); Torr. \& Gray, Fl. N. Amer., vol. 1, p. 213; Engl. in Mart., Fl. Bras., vol. 12, pt. 2, p. 71; Urb., Symb. Ant., vol. 4, p. 315. Tribulus maximus L., Sp. pl., vol. 1, p. 386 (1753). - El Valle, Miller \& Johnston, no. 7, July 12; Juan Griego pond, Johnston, no. 292, Aug. 14. Venezuela: near Cumaná, HBK., Nov. gen. et sp., vol. 6, p. 11. Further distribution, tropical America.

\section{Tribulus L.}

T. terrestris L., Sp. pl., vol. 1, p. 387 (1753), var. Cistoides (L.) Oliv., Hl. trop. Afr., vol. 1, p. $28+$ (1S6S); Urb., Symb. Ant., vol. 4, p. 315. T. cistoides L., Sp. pl., vol. 1, p. 387 (1753).- Santa Ana, 
Ernst; El Valle, Miller \& Johnston, no. 98, July 20; Johnston, no. 32, Aug. 3. Distribution general in tropies.

\section{RUTACEAE.}

AwYris P. Br.

A. maritma Jacq., Enum. pl. Carib., p. 19 (1760); Griseb., Fl. Brit. W. Ind., p. 174; Sargent, Silva N. Amer., vol. 1, p. 85, pl. 36.San Juan Mt., alt. 500 m., Johnston, no. 277, Aug. 28. Further distribution, tropical America.

\section{Citrus L.}

C. Aurantium L., Sp. pl., vol. 2, p. 782 (1753); Desc., Ant., vol. 1, p. 181, pl. 3S.-El Valle, Miller \& Johnston, no. 39, July 11. Cultivation general in tropical and sub-tropical countries.

\section{Esenbeckia HBK.}

E. pilocarpoides HBK., Nov. gen. et sp., vol. 7, p. 24S, pl. 655 (1825).- El Valle, Miller \& Johnston, no. 226, July 25. Venezuela: near Quetepe, HBK., l.c. Distribution general in tropical America.

\section{Zaxthoxylui L.}

Z. Pterota (L.) HBK., Nov. gen. et sp., vol. 6, p. 3 (1S23). Fagara Pterota L., Syst. nat., ed. 10, vol. 2, p. 897 (1759). F. lentiscifolia Griseb., Fl. Brit. W. Ind., p. 137 (1859), not Willd.--Santa Ana, Ernst. Further distribution, southern United States, West Indies to Trinidad.

\section{SIMARUBACEAE.}

\section{Castela Turp.}

C. Depressa Turp., Ann. mus. Par., vol. 7, p. 79, pl. 5 (1806).Juan Griego, Ernst. Further distribution, San Domingo.

C. Nicholsoni Hook., Bot. mise., vol. 1, p. 271, pl. 55 (1830). C. erecta Griseb., Fl. Brit. W. Ind., p. 140 (1859), not Turp.-- El Valle, Miller \& Johnston, no. 236, July 18; Pt. Moreno, Johnston, no. 118, July 8. Further distribution, Texas, Antigua. 


\section{Striana L.}

S. Maritina L., Sp. pl., vol. 1, p. 284 (1753); Griseb., Fl. Brit. IV. Ind., p. 58.- Juan Griego, Ernst; Pt. Mosquito, Johnston, no. 2S4, Aug. 10. Distribution general in tropics.

\section{BURSERACEAE.}

\section{Bursera Jacq.}

B. simaruba (L.) Sarg., Gard. and for., rol. 3, p. 260 (1S90) and Silva N. Amer., vol. 1, p. 97, pls. 41, 42; Lrb., Symb. Ant., vol. 4, p. 324.- South Hill, El Valle, alt. 270 m., Johnston, no. 270, July 3. Venezuela: Ernst, Sobre la flora y fauna, p. 226. Further distribution, West Indies, Panama. Tree, about $7 \mathrm{~m}$. high; wood odorous and bitter.

\section{MELIACEAE.}

\section{Melia L.}

M. Azedarach L., Sp. pl., vol. 1, p. $3 \$ 4$ (1753); Urb., Symb. Ant., vol. 4, p. 325.-Cultivated, El Valle, Miller \& Johnston, no. 112, July 1S. Venezuela: valley of Aragua, HBK., Nor. gen. et sp., vol. 5, p. 218. Distribution general in tropical countries.

\section{Trichilla P. Br.}

T. HirTa L., Syst. nat., ed. 10, vol. 2, p. 1020 (1759); Griseb., Fl. Brit. W. Ind., p. 129. T. spondiodes Jacq., Enum. pl. Carib., p. 20 (1760).- Santa Ana, Emst. Further distribution, Jamaica, Cuba.

\section{MALPIGHIACEAE.}

\section{HETEROPTERIS HBK.}

H. LAtrifolia (L.) Juss., Ann. sci. nat, bot., ser. 2, vol. 13, p. 276 (1S40); Griseb., Fl. Brit. W. Ind., p. 119. Banisteria laurifolia L., Sp. pl., ed. 2, vol. 2, p. 611 (1762).- South Hill, El Valle, alt. 300 m., Miller \& Johnston, no. 46, Aug. 1; Juan Griego trail, alt. 300. m., Johnston, no. 54, Aug. 14. Further distribution, Jamaica, Cuba, and Porto Rico.

H. purpurea (L.) HBK., Nor. gen. et sp., vol. 5, p. 164 (1S21), 
in obs.; Griseb., Fl. Brit. IT. Ind., p. 119. Banisteria purpurea L., Sp. pl., vol. 1, p. 427 (1753).-El Valle, climbing over a hedge of Clerodendron molle, Johnston, no. 55, July 9. Flowers light purple or pink. Venezuela: near Cumaná, HBK., Nov. gen. et sp., vol. 5, p. 164. Further distribution, West Indies and Venezuela.

\section{Malpighia L.}

M. GLabra L., Sp. pl., rol. 1, p. 425 (1753); Griseb., Fl. Brit. W. Ind., p. 116.- Juan Griego and Santa Ana, Ernst. Further distribution, tropical America.

M. Punicifolia L., Sp. pl., ed. 2, vol. 1, p. 609 (1762), var. vulGaris Ndz., Gen. Malpigh., p. S (1S99).-- El Valle, Miller \& Johnston, no. 253, Aug. 11; Johnston, no. 130, Aug. 30. Distribution general in tropical America.

\section{POLYGALACEAE.}

\section{SECURIDaCa L.}

S. cordata Johnston, Proc. Amer. acad. arts and sci., vol. 40, p. $6 S S$ (1905).- North Hill, El Valle, alt. 250 m., Johnston, no. 60, Aug. S. Endemic.

\section{EUPHORBIACEAE.}

\section{ACALYPHA L.}

A. macrostachy Jacq., Hort. Schoenb., rol. 2, p. 63, pl. 245 (1797); Griseb., Fl. Brit. WV. Ind., p. 47.--San Juan MI., alt. 400 m.; Johnston, no. 122, July 6 . A single group of this species was found growing among Heliconia Bihai. Distribution general. in northern South America.

\section{ADELIA L.}

A. Ricinella L., Syst. nat., ed. 10, vol. 2, p. 1298 (1759), and Pl. Jam. Pugill., p. 29; Browne, Jam., pl. 36, fig. 3.- Santa Ana, Emst. Further distribution, Jamaica, Cuba.

\section{Argithamia Sw.}

A. Candicaxs Sw., Prod. veg. Ind. Occ., p. 39 (17S8); Griseb., Fl. Brit. W. Ind., p. 44; Juss., Euph., pl. 7.- Juan Griego and Santa Ana, Ernst. Further distribution, West Indies and Caribbean Ids. 
A. erubescens Johnston, Proc. Amer. acad. arts and sci., vol. 40, p. 689 (1905).- El Valle, Miller \& Johnston, no. 213, July 22; Johnston, no. 58, Aug. 21. Endemic. Plate 30, figs. 4, 4a-c, 5, 5a-c.

\section{Croton L.}

C. chamaedrifolius Lam., Encycl., vol. 2, p. 215 (1786); Griseb., Fl. Brit. W. Ind., p. 41; Muell. Arg. in Mart., Fl. Bras., vol. 11, pt. 2, p. 271; Desc., Ant., vol. 7, p. 491.- Santa Ana, Ernst. Further distribution, West Indies, Panama, Guiana.

C. Flavens L., Syst. nat., ed. 10, rol. 2, p. 1276 (1759), and Pl. Jam. Pugill., p. 28; Griseb., Fl. Brit. W. Ind., p. 38.-El Valle, Miller \& Johnston, no. 38, July 15; Johnston, no. 45, Aug. S. Further distribution, Jamaica, Trinidad, St. Thomas.

C. Glandulosus L., Syst. nat., ed. 10, vol. 2, p. 1275 (1759); Griseb., Fl. Brit. W. Ind., p. 41; Muell. Arg. in Mart., Fl. Bras., vol. 11, pt. 2, p. 267; Jacq., Ic., vol. 1, p. 104; Urb., Symb. Ant., vol. 4, p. 343.- Hillside, El Valle, Miller \& Johnston, no. 5, July 11. Further distribution, temperate and tropical America.

C. helicoideus Muell. Arg., Limnaea, vol. 34, p. 97 (1865-66).El Valle, Miller \& Johnston, no. 232, July 30; Johnston, no. 339, Aug. 8. Found also in St. Vincent.

C. Lobatus L., Sp. pl., vol. 2, p. 1005 (1753); Griseb., Fl. Brit. IV. Ind., p. 42; Muell. Arg. in Mart., Fl. Bras., vol. 11, pt. 2, p. 237; Urb., Symb. Ant., vol. 4, p. 343.- Santa Ana, Emst; El Valle, Miller \& Johnston, no. 120, July 16 . Venezucla: near Bordones, HBK., Nov. gen. et sp., vol. 2, p. 90 . Distribution general in tropical America.

C. margaritensis Johnston, Proc. Amer. acad. arts and sci., vol. 40, p. $6 \$ 9$ (1905).- Among shrubs near summit of San Juan Mt., alt. $700 \mathrm{~m}$., Johnston, no. 50, Aug. 28. Endemic. Plate 30, figs. 3 and $3 \mathrm{a}$.

C. Milleri Johnston, Proc. Amer. acad. arts and sci., vol. 40, p. 690 (1905).-El Valle, Miller \& Johnston, no. 229, July 18; Johnston, no. 48, Aug. 10. Common on the plains between El Valle and Pt. Mosquito. Endemic. Plate 30, figs. 2, 2a-ll.

C. Niveus Jacq., Enum. pl. Carib., p. 32 (1760); Griseb., Fl. Brit. IV. Ind., p. 40.- Santa Ana, Ernst; El Valle, Miller \& Johnston, no. 107, July 20. Further distribution, Jamaica, Dominica, Colombia.

C. oralifolius Vahl in West, Bidr. Ste. Croix, p. 307 (1793); 
Griseb., Fl. Brit. IV. Ind., p. 41.- Santa Ana, Ernst; El Valle, Miller \& Johnston, no. 199, July 14; Johnston, no. 46, Aug. 31. Further distribution, West Indies and Venezuela.

C. Populifolius Mill., Gard. dict., ed. S, no. 7 (1768), as Populi folia; Griseb., Fl. Brit. W. Ind., p. 41.-San Juan Mt., alt. 500 m., Johnston, no. 47, Aug. 28. Further distribution, West Indies.

C. Pseudochina Schlecht., Linnaea, rol. 5, p. $\$ 4$ (1830), as PseudoChina; Griseb., Fl. Brit. W. Ind., p. 39.- Santa Ana, Ernst. Further distribution, tropiçal America.

\section{Euphorbia L.}

E. Buxifolia Lam., Encycl., vol. 2, p. 421 (17S6); Griseb., Fl. Brit. W. Ind., p. 58 ; Muell. Arg. in Mart., Fl. Bras., vol. 11, pt. 2, p. 681.- Juan Griego, Ernst; Pt. Mosquito, Johnston, no. 44, Aug. 10. Found growing on the dunes of shifting sands. Further distribution, tropical America.

E. hypericifolia L., Sp. pl., vol. 1, p. 454 (1753); Griseb., Fl. Brit. IV. Ind., p. 5t; Muell. Arg. in Mart., Fl. Bras., vol. 11, pt. 2, p. 677.-El Valle, Miller \& Johnston, no. 109, July. Var. LAsioCARPA Griseb., l. c., p. 54 (1S59). E. lasiocarpa Klotzsch, Nov. act. nat. cur., vol. 19, suppl. 1, p. 414 (1843).- Santa Ana, Ernst. Venezuela: near Cumaná, HBK., Nor. gen. et sp., vol. 2, p. 56 (typical form); Loefling, Reise nach den spanischen Ländern, p. 152153. Common in tropical countries.

E. petiolaris Sims, Bot. mag., vol. 23, pl. $\$ 83$ (1806).- El Valle, Miller \& Johnston, no. 235, July 1S. Also from Island of St. Thomas.

E. Pilulifera L., Sp. pl., vol. 1, p. 454 (1753); Griseb., Fl. Brit. W. Ind., p. 54; Muell. Arg. in Mart., Fl. Bras., vol. 11, pt. 2, p. 684.El Valle, Miller \& Johnston, no. 108, July. Common in tropical countries.

E. тhymifolia L., Sp. pl., vol. 1, p. 454 (1753); Burm. f., Fl. Ind., p. 112; Muell. Arg. in Mart., Fl. Bras., vol. 11, pt. 2, p. 6st. E. macnlata Griseb., Fl. Brit. W. Ind., p. 53, not L.- El Valle, Miller \& Johnston, no. 150, Aug. 9; Juan Griego, at the pond and on the seashore, Johnston, no. 251, Aug. 14. Widespread in tropical countries.

\section{Hipponiane L.}

H. Mancinella L., Sp. pl., vol. 2, p. 1191 (1753); Griseb., Fl. Brit. IT. Ind., p. 50.- Juan Griego, Ernst; Pt. Moreno, Johnston, no. 261, 
July 13. Venezuela: Cumaná, Loefling, Reise nach den spanischen Ländern, p. 152-153. Further distribution, Cuba to Venezuela and Panama.

\section{Hura L.}

H. Crepitans L., Sp. pl., vol. 2, p. 1006 (1753); Griseb., Fl. Brit. W. Ind., p. 50; Muell. Arg. in Mart., Fl. Bras., vol. 11, pt. 2, p. 632.Asuncion, Ernst. Further distribution, Cuba, Brazil.

\section{JATROPHA L.}

J. Curcas L., Sp. pl., vol. 2, p. 1006 (1753); Griseb., Fl. Brit. W. Ind., p. 36; Muell. Arg. in Mart., Fl. Bras., vol. 11, pt. 2, p. 487.Santa Ana, Emst; El Valle, Miller \& Johnston, no. 237, July 24. Venezuela: Nova Andalusia, HBK., Nov. gen. et sp., vol. 2, p. 104. Distribution general in tropics.

J. gossypifolia L., Sp. pl., vol. 2, p. 1006 (1753); Griseb., Fl. Brit. W. Ind., p. 36; Muell. Arg. in Mart., Fl. Bras., vol. 11, pt. 2, p. 491; Urb., Symb. Ant., vol. 4, p. 350.-Santa Ana, Ernst; El Valle, Miller \& Johnston, no. 27, July 11. Venezuela: near Cumaná and La Guaira, HBK., Nov. gen. et sp., rol. 2, p. 10t. Distribution general in tropical America.

J. URens L., Sp. pl., vol. 2, p. 1007 (1753), var. Stinulosi (Michx.) Muell. Arg. in DC., Prod., vol. 15, pt. 2, p. 1101 (1S62). J. stimulosa Michx., Fl., vol. 2, p. 216 (1S03)._-Santa Ana, Ernst; El Valle, Miller \& Johnston, no. 48, July S; Johnston, no. 57, Aug. 3. Venezuela: near Gumaná, HBK., Nov. gen. et sp., rol. 2, p. 106. La Guaira, Robinson \& Lyon, July 12, 1900. Common in American tropics.

\section{MaNiHo't Adans.}

M. utilissma Pohl, Pl. Bras. Ic., vol. 1, p. 32, pl. 24 (1827); Muell. Arg. in Mart., Fl. Bras., vol. 11, pt. 2, pp. 457, 707; Urb., Symb. Ant., rol. 4, p. 350. Janipha Manihot HBK., Nor. gen. et sp., vol. 2, p. 108 (1817); Griseb., Fl. Brit. W. Ind., p. 37.- El Valle, Miller \& Johnston, no. 102, July 24. Widely cultivated in the hills and on the plain by Asuncion. Commonly cultivated in American tropies and subtropics.

\section{Pedilanthus L.}

P. Tithymaloides (L.) Poit., Ann. mus. Par., vol. 19, p. 390, pl. 19 (1S12); Griseb., Fl. Brit. W. Ind., p. 52. Euphorbia tithymaloides 
L., Sp. pl., vol. 1, p. 453 (1753).- Santa Ana, Ernst; El Valle, Miller \& Johnston, no. 31, July 10; Johnston, no. 59, July 27. Slender shrub, abour $1 \mathrm{~m}$. high, with red flowers. Venezuela: Ernst, Sobre la flora y fauna, p. 226; between Bordones and Cumaná, HBK., Nov. gen. et sp., vol. 2, p. 63; La Guaira, Robinson \& Lyon, July 6, 1900. Further distribution, West Indies.

\section{Phyllanthus L.}

P. Niruri L., Sp. pl., vol. 2, p. $9 \$ 1$ (1753); Griseb., Fl. Brit. W. Ind., p. 34; Muell. Arg. in Mart., Fl. Bras., vol. 11, pt. 2, p. 53, 705.Santa Ana, Ernst; Juan Griego trail, alt. 400 m., Johnston, no. 140, Aug. 14. Further distribution, tropical countries.

\section{Ricinus L.}

R. communis L., Sp. pl., vol. 2, p. 1007 (1753); Griseb., Fl. Brit. IV. Ind., p. 37; Muell. Arg. in Mart., Fl. Bras., vol. 11, pt. 2, p. 420.Santa Ana, Ernst. Venezuela: Cumaná, Loefling, Reise nach den spanischen Ländern, p. 152-153. Further distribution, tropies.

\section{Sebastiania Spreng.}

S. Corniculata (Juss.) Muell. Arg. in DC., Prod., vol. 15, pt. 2, p. 1168 (1862) and in Mart., Fl. Bras., vol. 11, pt. 2, p. 551. Microstachys corniculata Juss., Euphorb. Tent., p. 49 (1824); Griseb., Fl. Brit. W. Ind., p. 49.- Margarita according to Ernst. Further distribution, Trinidad and northern South America.

\section{Tragia L.}

'T. volubicis L., Sp. pl., vol. 2, p. 980 (1753); Griseb., Fl. Brit. IV. Ind., p. 4S; Muell. Arg. in Mart., Fl. Bras., vol. 11, pt. 2, p. 412.Santa Ana, Ernst; El Valle, Miller \& Johnston, no. 15, July 24; Juan Griego trail, Johnston, no. 253, July 31. Further distribution, West Indies and tropical South America.

\section{Genera Euphorbiacearum adHUC indeterminata.}

Euphorbiacea Actinostemoni affinis.-Johnston, no. 332 and 333, alt. $400 \mathrm{~m}$., July 29.

EupHorbiaceA Argithamniae affinis.- Miller \& Johnston, no. 251, Aug. 3. 


\section{ANACARDIACEAE.}

\section{Anacardium L.}

A. occidentale L., Sp. pl., vol. 1, p. 383 (1753); Jacq., Hist. Stirp. Am., p. 124; Griseb., Fl. Brit. W. Ind., p. 176.- Reported by Ernst. Cultivated in the Asuncion valley. Distribution general in tropical countries.

\section{Mangifera L.}

M. INDica L., Sp. pl., vol. 1, p. 200 (1753); Griseb., Fl. Brit. W. Ind., p. 176.- Cultivated extensively. Common to all tropical countries.

\section{MaUria Kunth.}

M. Heterophylla HBK., Nov. gen. et sp., vol. 7, p. 13, pl. 606 (1S25).- Juan Griego and Santa Ana, Ernst. Venezuela: Colonia Tovar, Fendler, no. 174, 1S56-57. Further distribution, Colombia and Peru.

\section{Spondias L.}

S. Lutea L., Sp. pl., ed. 2, vol. 1, p. 613 (1762); Griseb., Fl. Brit. IV. Ind., p. 175.-Cultivated according to Ernst. In the market place of El Valle also. Venezuela: Cumaná, Loefling (1754), Reise nach den spanischen Ländern, p. 152-153. Common to all tropical countries.

\section{CELASTRACEAE.}

\section{Elaeodendron Jacq.}

E. sP. Johnston, no. 307. Related to E. xylorarpum DC., Prod., vol. 2, p. 11, from St. Thomas. Similar to no. 926 of P. Sintenis's Plantae Portoricenses (1S85) determined by I. Urban. Differs in having broader leaves, which are often orbicular and very broad at the base. In general the leaves are larger than in E. xylocarpum.

\section{Mrginda Jacq.}

M. Rhacoma Sw., Prodl. reg. Ind. Occ., p. 39 (17S8); Griseb., Fl. Brit. W. Ind., p. 146.- Juan Griego, Emst. Tenezuela: near Laguna Chica, HBK., Nov. gen. et sp., vol. 7, p. 67. Florida, Jamaica, Cuba. 
M. sp. San Juan Mt., alt. 600 m., Johnston, no. 282, Aug. 28. Allied to M. latifolia Sw. and M. Grisebachii Sarg. Leaves in this form larger than those of the others, obovate, entire, sometimes $5 \mathrm{~cm}$. long and $3 \mathrm{~cm}$. wide, the base often obtuse, decurrent into a short petiole, the apex rounded, obtuse or minutely retuse; flowers on the plan of four.

\section{SAPINDACEAE.}

\section{Cardiospermum L.}

C. Halicacabun L., Sp. pl., vol. 1, p. 366 (1753); Griseb., Fl. Brit. W. Ind., p. 122; Desc., Ant., vol. 4, pl. 241.- Santa Ana, Ernst; El Valle, Miller \& Johnston, no. 208, July 20, Venezuela: Ernst. Sobre la flora y fauna, p. 226. Common in tropical countries.

\section{MeLICOCCA L.}

M. Bı.JugA L., Sp. pl., ed. 2, vol. 1, p. 495 (1762); Griseb., Fl. Brit. W. Ind., p. 127.- El Valle, Miller \& Johnston, no. 12S, July 18. Further distribution, tropical America.

\section{Paullinia L.}

P. Cururu L., Sp. pl., vol. 1, p. 365 (1753); Desc., Ant., vol. 3, pl. 181.- El Valle, Miller \& Johnston, no. 239, July 30; Tacarigua, Johnston, no. 66, Aug. 14. Distribution general in IVest Indies. Not the same as Serjania nodosa as claimed by. some authors, for the fruit is pyriform and not at all alate.

\section{SAPINDUS L.}

S. SAponaria L., Sp. pl., vol. 1, p. 367 (1753); Griseb., Fl. Brit. IV. Ind., p. 126.- Santa Ana, Ernst; El Valle, Miller \& Johnston, no. 127, July 18. Further distribution, Jamaica, and Venezuela.

Talisia Aubl.

T. olivaeformis (HBK.) Radlk., Sitzb. math.-phys. Akad., Muench., vol. S, p. 342 (1878). Melicocca olivaeformis HBK., Nov. gen. et sp., vol. 5, p. 130 (1821). - Santa Ana, Ernst; EI Valle, alt. 300 m., Johnston, no. 299, July 4. Further distribution, Colombia.

\section{Urvillea HBK.}

U. UlilaceA HBK., Nor. gen. et sp., rol. 5, p. 106, pl. 440 (1821).El Valle, Miller \& Johnston, no. 23, July 31. Venezuela: near Caracas, HBK, l. c. Further distribution, tropical America. 


\section{RHAMNACEAE.}

\section{Ziziphus Juss.}

Z. sp.- El Valle, River trail, Johnston, no. 269, July 15. A tree, about $7 \mathrm{~m}$. high, with wide-spreading top. Related to Z. mexicana Rose, Contrib. U. S. nat. herb., vol. 1, p. 315. Differs in having leaves more broadly oval, more rounded at base, and distinctly threenerved. The margin is almost the same in each.

\section{VITACEAE.}

\section{Cissus L.}

C. sicyoides L., Syst. nat., ed. 10, vol. 2, p. 897 (1759); Griseb., Fl. Brit. IV. Ind., p. 102; Desc., Ant., vol. 7, pl. 481.- Santa Ana, Ernst; El Valle, Miller \& Johnston, no. 244, Aug. 1. Further distribution, tropical America.

\section{'TILIACEAE.}

\section{Corchorus L.}

C. Acutangulus Lam., Encycl., vol. 2, p. 104 (1786); Wight, Ic. pl. Ind. Or., rol. 3, p. 739; Griseb., Fl. Brit. WV. Ind., p. 97.-- El Valle, Miller \& Johnston, no. 97, July 11. Common to all tropical countries.

C. Hirsutus L., Sp. pl., vol. 1, p. 530 (1753); Jacq., Hort. Vind., vol. 3, pl. 57; Griseb., Fl. Brit. W. Ind., p. 97.- Juan Griego, Ernst. Further distribution, Antigua, Guadeloupe, Venezuela.

C. siliquosus L., Sp. pl., vol. 1, p. 529 (1753); Jacq., Hort. Vind., vol. 3, pl. 59; Griseb., Fl. Brit. W. Ind., p. 97.- Santa Ana, Ernst. Further distribution, southern United States and tropical America.

\section{Triumfetta L.}

T. Lappula L., Sp. pl., vol. 1, p. 444 (1753); Griseb., Fl. Brit. IV. Ind., p. 95.- Santa Ana, Ernst. Venezuela: Colonia Tovar, Fendler, no. 1918, 1856-57. Further distribution, Mexico, IVest Indies Panama, and Cape Verde Ids. 


\section{MALVACEAE.}

\section{Abutilon Hill.}

A. CRispuir (L.) G. Don, Gen. syst., vol. 1, p. 502 (1831); Wight, Ic. pl. Ind. Or., vol. 1, pl. 68; Griseb., Fl. Brit. IV. Ind., p. 79; Schumann in Mart., Fl. Bras., vol. 12, pt. 3, p. 382. Sida crispa L. Sp. pl., vol. 2, p. 685 (1753).-El Valle, Miller \& Johnston, no. 71; Juan Griego, Johnston, no. 271, Aug. 14. Venezuela: Colonia Tovar, Fendler, no. 97, 1851-55. Common in tropics.

A. unibellatum (L.) Sweet, Hort. Brit., ed. 1, p. 53 (1827); Griseb., Fl. Brit. IT. Ind., p. 7S. Sida umbellata L., Syst. nat., ed. 10, vol. 2, 1145 (1759); Jacq., Hort. Vind., vol. 1, pl. 56.-El Valle, Miller \&. Johnston, no. 152, July 7. Further distribution, Jamaica, St. Thomas.

\section{BASTARDIA HBK.}

B. viscosa HBK., Nor. gen. et sp., vol. 5, p. 256 (1821); Griseb., Fl. Brit. WV. Ind., p. S0; Schumann in Mart., Fl. Bras., vol. 12, pt. 3, p. 360.-Asuncion, Ernst. Venezuela: near Cumaná, HBK., l. c.; Colonia Tovar, Fendler, no. 2543, 1856-57. Distribution, Cuba to Brazil and Peru.

\section{Cienfeggosia Cav.}

C. Heterophyla (Vent.) Garcke in Bonplandia, vol. S, p. 150 (1S60). Redutea heterophylla Vent., Descr. pl. jard. Cels, pl. 11 (1S00). - El Valle, Miller \& Johnston, no. 66, July 5. Further distribution, tropical America.

\section{Gossypiun L.}

G. Barbadense L., Sp. pl., vol. 2, p. 693 (1753); Griseb., Fl. Brit. IV. Ind., p. S6; Gürke in Mart., Fl. Bras., vol. 12, pt. 3, p. 582.-E] Valle, Miller \& Johnston, no. 207, July 31. Tenezuela: Cumaná, Loefing, 1754. Common in tropical America.

\section{Malvastrum Gray.}

M. spicatuir Gray, Mem. Amer. acad. arts and "sci., n. s., vol. t, p. 22 (1S49); Griseb., Fl. Brit. WV. Ind., p. 72; Schumann in Mart., Fl. Bras., vol. 12, pt. 3, p. 271.-Santa Ana, Ernst. Venezuela: Colonia Tovar, Fendler, no. 11S, 1S5̃ -55. Further distribution, tropical countries. 


\section{Pavonia Cav.}

P. spinifex Cav., Diss., vol. 3, p. 133, pl. 45 (1787); Griseb., Fl. Brit. W. Ind., p. 82; Gürke in Mart., Fl. Bras., vol. 12, pt. 3, p. 480.Juan Griego, Ernst. Venezuela: near Caripe, HBK., Nov. gen. et sp., vol. 5, p. 280. Further distribution, tropical America.

\section{SiDA L.}

S. ACUTA Burm. f., Fl. Ind., p. 147 (1768); Schumann in Mart., Fl. Bras., vol. 12, pt. 3, p. 325. S. carpinifolia L. f., Suppl., p. 307 (1781); Griseb., Fl. Brit. W. Ind., p. 73.-Santa Ana, Ernst. Distribution general in tropical countries.

S. Clliaris L., Syst. nat., ed. 10, vol. 2, p. 1145 (1759); Griseb., Fl. Brit. W. Ind., p. 73; Schumann in Mart., Fl. Bras., vol. 12, pt. 3, p. 283.- Santa Ana, Ernst; El Valle, Miller \& Johnston, no. 209, Aug. 9. Distribution general in tropical America.

S. rhombifolia L., Sp. pl., vol. 2, p. 684 (1753); Griseb., Fl. Brit. IT. Ind., p. 74; Schumann in Mart., Fl. Bras., vol. 12, pt. 3, p. 337.Santa Ana, Ernst. Venezuela: near Cumaná, Loefling, 1754; HBK., Nov. gen. et sp., vol. 5, p. 261. Found in tropical countries.

S. spinosa L., Sp. pl. 5, p. 261, vol. 2, p. 683 (1753), var. Angustifolia (Lam.) Griseb., Fl. Brit. W. Ind., p. 74 (1859); Schumann in Mart., Fl. Bras., vol. 12, pt. 3, p. 298. S. angustifolia Lam., Encycl., vol. 1, p. 4 (1783).--Asuncion, Miller \& Johnston, no. 6 , July 30 . Found in tropical countries.

\section{Thespesia Soland.}

T. populnea (L.) Soland. ex Correa in Ann. mus. Par., vol. 9, p. 290, pl. 8, fig. 2 (1807); Griseb., Fl. Brit. W. Ind., p. 87. Hibiscus populneus L., Sp. pl., vol. 2, p. 694 (1753).- Juan Griego, Emst. Further distribution, tropical countries.

\section{Wissadula Medic.}

IV. Periplocifolia (L.) Griseb., Cat. pl. Cuba, p. 25 (1866). Sida periplocifolia L., Sp. pl., vol. 2, p. 684 (1753).- El Valle, Miller \& Johnston, no. 126, Aug. 31. Further distribution, Jamaica. 


\section{BOMBACACEAE.}

BOMBax L.

B. cumanense HBK., Nov. gen. et sp., vol. 5, p. 300 (1821). - Santa Ana, Ernst. Venezuela: near Cumaná, HBK., l. c. There are several Bombax trees occurring in El Valle, but no specimens were collected.

\section{STERCULIACEAE.}

Guazuma Adans.

G. ulmifolia Lam., Encycl., vol. 3, p. 52 (17S9); Griseb., Fl. Brit. W. Ind., p. 91.- Santa Ana, Ernst. Distributed from Cuba to Brazil.

\section{Helicteres L.}

H. Baruensis Jacq., Enum. pl. Carib., p. 30 (1760), and Hist. Stirp. Am., p. 236; Schumann in Mart., Fl. Bras., vol. 12, pt. 3, p. 20.Santa Ana, Ernst. Venezuela: Vargas, Flora, p. 192. Further distribution, tropical America.

\section{Melochia L.}

M. tomentosa L., Syst. nat., ed. 10, vol. 2, p. 1140 (1759); Griseb., Fl. Brit. W. Ind., p. 93.- Santa Ana, Ernst; El Valle, Miller \& Johnston, no. 44, July 5; Juan Griego trail, Johnston, no. 56, July 31. Shrub, $1 \mathrm{~m}$. high ; flowers pink or purplish. Venezuela: near Cumaná, HBK., Nov. gen. et sp., vol. 5, p. 323; Caracas, Birschel; Colonia Tovar, Fendler, no. 22S9, 1856-57. Further distribution, tropical America.

\section{WALTHERIA L.}

W. Americana L., Sp. pl., vol. 2, p. 673 (1753); Griseb., Fl. Brit. IV. Ind., p. 95.- - El Valle, Miller \& Johnston, no. 202, July S. Venezuela: near Bordones and Cumaná, HBK., Nov. gen. et sp., vol. 5 , p. 333; Colonia Tovar, Fendler, nos. 110 and 112, 185t-55. Widely distributed in tropical countries.

\section{MARCGRAVIACEAE.}

\section{Caracasia Szýszyl.}

C. Tremadena (Ernst) Szyszyl. in Engl. et Prantl, Natürl. Pflanzenfam., vol. 3, pt. 6, p. $16+$ (1893). Vargasia tremadena Ernst, Vár- 
gas consid. como bot., p. 23 (1877).-Alt. 700 m., San Juan Mt., Johnston, no. 279, Aug. 28. A shrub, $2 \mathrm{~m}$. high. Venezuela: near Caracas, Ernst, May, 1876.

\section{GUTTIFERAE.}

\section{Cluesia L.}

C. Flava Jacq., Enum. pl. Carib., p. 34 (1760), and Hist. Stirp. Am., p. 272; Griseb., Fl. Brit. W. Ind., p. 107.- San Juan Mt., alt. 700 m., Johnston, no. 133, July 31. Occurs also in Jamaica.

\section{BIXACEAE.}

\section{Bixa L.}

B. Orellana L., Sp. p]., vol. 1, p. 512 (1753); Griseb., Fl. Brit. W. Ind., p. 20; Eichler in Mart., Fl. Bras., vol. 13, pt. 1, p. 433, pl. 87.- Santa Ana, Ernst; Juan Griego trail on open hillside, alt. 300 m., Johnston, no. 11, July 2. Shrub, 3-4 m. high.-- Venezuela: Cumaná, Loefling, 1754. Found in tropical countries.

\section{VIOLACEAE. \\ Hrbanthus Jacq.}

H. oppositifolius (L.), n. comb. Tiola oppositifolia L., Sp. pl., ed. 2, vol. 2, p. 1327 (1763). Ionidium oppositifolium Roem. \& Schult., Syst., vol. 5, p. 395 (1819). - El Valle, Miller \& Johnston, no. 124, July 6. Venezuela: Colonia Tovar, Fendler, no. 2419, 1S56-57; Cumaná, Loefling according to Roem. \& Schult., l. $c$.

\section{Rinorea Aubl.}

R. marginata (Tr. \& Planch.) Rusby in herb. Alsodeia marginata Triana et Planchon, Ann. sci. nat., bot., ser. 4, vol. 17, p. 127 (1862).-El Valle, Miller \& Johnston, no. 225, July 27; San Juan IIt., alt. 300 m., Johnston, no. 120, Aug. 2S. Further distribution, Colombia.

\section{SaUvagesia L.}

S. erecta L., Sp. pl., vol. 1, p. 203 (1753); Griseb., Fl. Brit. W. Ind., p. 112.- San Juan MIt., alt. 795 m., Johnston, no. 90, Aug. 28. 
Venezuela: near Caripe and Cumanacoa, HBK., Nov. gen. et sp., vol. 5, p. 389; Colonia Tovar, Fendler, no. 2319, 1856-57. Distribution general in tropics.

\section{FLACOURTIACEAE.}

\section{Casearia Jacq.}

C. PARvifolia Willd., Sp. pl., vol. 2, p. 628 (1799); Jacq., Hist. Stirp. Am., p. 127; Griseb., Fl. Brit. IV. Ind., p. 23.- South Hill, El Valle, Johnston, no. 129, Aug. 31. Distributed from Cuba to Guiana.

C. guianensis (Aubl.), n. comb. Iroucana guianensis Aubl., Pl. Guian., vol. 1, p. 329, pl. 127 (1775). Casearia ramiflora Vahl, Symb., vol. 2, p. 50 (1791); Griseb., Fl. Brit. W. Ind., p. 24.- El Valle, Miller \& Johnston, no. 19, Aug. 5. Distributed from Cuba to Bahia.

C. spiralis Johnston, Proc. Amer. acad. arts and sci., vol. 40, p. 691 (1905).- El Valle, River trail, Johnston, no. 2S3, Aug. 30. Endenic.

C. sylvestris Sw., Fl. Ind. Occ., vol. 2, p. 752 (1800). - El Valle, Miller \& Johnston, no. 105, July 18; San Juan Mt., alt. 500 m., Johnston, no. 266, July 19. Further distribution, tropical America.

\section{Irlosua Forst. f.}

X. Nitidua (Hellen.) Gray ex Griseb., Fl. Brit. IV. Ind., p. 21 (1859). Hisingera nitida Hellen., Vet. akad. handl. Stocklı., 1792, p. 32, pl. 2 (1792).- Santa Ana, Ernst. Distributerl in tropical America.

\section{TURNERACEAE.}

\section{TurNerA L.}

T. Diffusa Willd. ex Schult., Syst., rol. 6, p. 679 (1820).- El Valle, Miller \& Johnston, no. 266, July 30, and Johnston, no. 28S, July 15. Further distribution, Brazil, West Indies, and Central America.

T. ulmifolia L., Sp. pl., vol. 1, p. 271 (1753).-Asuncion, Miller \& Johnston, no. 223, July 30. Further distribution, tropical America. 


\section{PASSIFLORACEAE.}

\section{PAssiflora L.}

P. roetida L., Sp. pl., vol. 2, p. 959 (1753); Griseb., Fl. Brit. IV. Ind., p. 294.- Santa Ana, Ernst; El Valle, Miller \& Johnston, no. S5, July 9, and Johnston, no. 63, Aug. 3. Venezuela: Cumaná, Loefling, 1754; in Prov. Cumaná, in Sierra de Chacao, and near the town of Fernando de Apure, HBK., Nov. gen. et sp., vol. 2, p. 138; Colonia 'Tovar, Fendler, no. 475, 1854-55. Distribution general in tropical America.

P. LaUmifolia L., Sp. pl., vol. 2, p. 956 (1753); Griseb., Fl. Brit. IV. Ind., p. 293.- Juan Griego trail, alt. 450 m., Johnston, no. 289, Aug. 12 to 15. Further distribution, tropical America.

P. nonticola Johnston, Proc. Amer. acad. arts and sci., vol. 40, p. 692 (1905).- Climbing over low shrubs at the mountain top, alt. 700 to 795 m., San Juan Mt., Johnston, no. 64, July 11. Endemic.

P. Nitexs Johnston, Proc. Amer. acad. arts and sci., vol. 40, p. 692 (1905). - El Valle, South Hill, Johnston, no. 65, Aug. 31. Endemic.

\section{CARICACEAE.}

\section{Carica L.}

C. Papaya L., Sp. pl., vol. 2, p. 1036 (1753); Griseb., Fl. ${ }^{\top}$ Brit. IV. Ind., p. 290.- In Asuncion valley. Venezuela: Cumaná, Loefling, Reise nach den spanischen Ländern, p. 152-153. Cultivated in all tropical countries.

\section{LOASACEAE.}

\section{Mentzelia L.}

M. aspera L., Sp. pl., vol. 1, p. 516 (1753); Griseb., Fl. Brit. IV. Ind., p. 298.- Juan Griego, Ernst. Venezuela: Colonia 'Tovar, Fendler, no. 453, 1856-57. Further distribution, in tropical America.

\section{BEGONIACEAE.}

\section{Begonia L.}

B. scandexs Sw., Prod., p. 86 (17SS).- Juan Griego trail, alt. 400 m., Jolnston, no. 291, July 31. Further distribution, West Indies, Brazil, Peru. 


\section{CACTACEAE.}

\section{Cereus Mill.}

C. Caripensis (HBK.) DC., Prod., vol. 3, p. 467 (1828). Cactus caripensis HBK., Nov. gen. et. sp., vol. 6, p. 66 (1S23).- South Hill, El Valle, alt. $300 \mathrm{~m}$., Johnston, no. 217, July 18. Further distribution, Mexico.

C. Eburneus Salm-Dyck, Obs. bot., p. 6 (1S22).-El Valle, Johnston, no. 342, July 27. Further distribution, Curaçoa and Chili.

C. Jaliacaru DC., Prod., rol. 3, p. 467 (182S).- Tacarigua, Johnston, no. 341, Aug. 15. Further distribution, Brazil.

C. Margaritensis Johnston, Proc. Amer. acad. arts and sci., rol. 40, p. 693 (1905). - El Valle, on the hillside, Johnston, no. 344, July 27. Endemic.

C. Swartzn Griseb., Fl. Brit. IV. Ind., p. 301 (1860).- Santa Ana, Ernst. Found also in Jamaica.

\section{Mammillaria Haw.}

M. simplex Haw., Sin. pl. succ., p. 177 (1812); Griseb., Fl. Brit. IV. Ind. p. 300.- Juan Griego, Ernst. Further distribution, Haiti and Tenezuela.

\section{Melocactus Link \& Otto.}

II. comuvis Link \& Otto in Verh. preuss. Ver. Gartenb., vol. 3, p. 417, pl. 11 (1S27); Griseb., Fl. Brit. W. Ind., p. 300.-Juan Griego, Emst; El Valle, plain by sea, Miller \& Johnston, no. 272, Aug. 3; Pt. Moreno, Johnston, no. 343, Aug. 10. Further distribution, West Indies.

\section{Opuntia Mill.}

O. Leptocaulis DC. in Mém. mus. Par., vol. 17, p. 118 (1828).En route El Valle to San Antonio, Johnston, no. 340, Aug. 3. Further distribution, Mexico.

O. 'Tuna Mill., Gard. dict., ed. \&, no. 3 (1768); Griseb., Fl. Brit. W. Ind., p. 302.-Common ererywhere on the plains and hillsides. El Valle, Johnston, no. 218, July 4. Common in tropical America.

\section{Pereskia Mill.}

P. opuntiaeflora DC. in Mém. mus. Par., vol. 17, p. 76, p . 19 (1828).- Pt. Moreno, Johnston, no. 216. Further distribution, Mexico. 
P. sP., reported by Emst at Santa Ana, is probably the above.

Rhipsalis Gaertn.

R. Cassutha Gaertn., Fruct., vol. 1, p. 137, pl. 28 (1788); Griseb., Fl. Brit. W. Ind., p. 302 as cassytha.-El Valle, Johnston, no. 16. Found hanging from the branches of trees; fruit becoming white. Further distribution, tropical America.

\section{THYMELAEACEAE. \\ DaphNopsis Mart. \& Zuce.}

D. americana (Mill.), n. comb. Laurus americana Mill., Dict., ed. S, no. 10.(1768). Daphne tinifolia Sw., Prod. veg. Ind. Occ., p. 63 (1788). Daphnopsis tinifolia Griseb., Fl. Brit. W. Ind., p. 278 (1860).- Juan Griego trail, alt. 450 m., Johnston, no. 257, Aug. 14. Further distribution, Jamaica.

\section{LI'THRACEAE.}

\section{Rotala L.}

R. Dextifera (Gray) Koehne in Engl., Bot. Jahrb., vol. 1, p. 161 (1881). Ammannia dentifera Gray, Pl. Wright., vol. 2, p. 55 (1853). — Juan Griego, Johnston, no. 276, Aug. 14. Further distribution, Mexico.

\section{RHIZOPHORACEAE.}

\section{Rhizophora L.}

R. Maxgle L., Sp. pl., vol. 1, p. 443 (1753); Griseb., Fl. Brit. W. Ind., p. 274.- Laguna Chica, Johnston, no. 246, Aug. 10, also at Pt. Piedras, and at Laguna Grande. Tenezuela: Ernst, Sobre la flora y fauna, p. 223. Common to tropical countries.

\section{IIRTACEAE.}

\section{Mrrela DC.}

M. Coriacea (Vahl) DC., Prod., vol. 3, p. 243 (1828); Griseb., Fl. Brit. W. Ind., p. 234. Myrtus coriacea Vahl., Symb., vol. 2, p. 59 (1791).-San Juan Mt., alt. 780 m., Johnston, no. 263, July 6. These 
specimens differ from the typical form in having oral leares with short blunt points, and in having narrow calyx-lobes. Further distribution, West Indies.

\section{Psidiuli L.}

P. GUajava L., Sp. pl., vol. 1, p. 470 (1753). P. Guara Radd. acc. to Griseb., Fl. Brit. IV. Ind., p. 241 (1560).- El Valle, Miller \& Johnston, no. 140, July 14, and Johnston, no. 241, July 2. Venezuela: Cumaná, Loefling Reise nach deli spanischen Ländern, p. 152-153. Common to tropical countries.

\section{COMBRETACEAE.}

\section{Combretum L.}

C. SEcunduy Jacq., Enum. pl. Carib., p. 19 (1760).- Santa Ana, Ernst. Further distribution, Trinidad, Tenezuela, Colombia, Guiana.

\section{CoNocarpes L.}

C. erectus L., Sp. pl., vol. 1, p. 176 (1753); Jacq., Hist. Stirp. Am., p. 78; Griseb., Fl. Brit. W. Ind., p. 277.- Juan Griego, Ernst; Laguna Chica, Johnston, no. 215, Aug. 10. Further distribution, tropical America and tropical Africa.

\section{LAGUNCULARIA Gaertn.}

L. RACEMosa Gaertn. f., Fruct., vol. 3, p. 209, pl. 217, fig. 3 (1805); Griseb., Fl. Brit. W. Ind., p. 276.- Juan Griego, Ernst; Laguna Chica, Johnston, no. 247, Aug. 10. Further distribution, tropical America and tropical Africa.

\section{Quisqualis L.}

Q. INDICA L., Sp. pl., ed. 2, vol. 1, p. 556 (1762).—EI Valle, Miller \& Johnston, no. 91, July 29. Cultivated in Margarita from tropical Asia.

\section{Terminalia L.}

T. Brceras (L.) Wright in Sauv., Fl. Cub., p. 38 (1873). Bucida Buceras L., Syst. nat., ed. 10, vol. 2, p. 1025 (1759).- San Juan Mt., Johnston, no. 275, Aug. 28. Further distribution, tropical America. 


\section{MELASTOMACEAE.}

\section{Blakea P. Br.}

B. Monticola Johnston, Proc. Amer. acad. arts and sci., vol. 40, p. 693 (1905).-- Abundant on the exposed top of San Juan Mt., alt. 700 to $795 \mathrm{~m}$., Johnston, no. 51, July 6 . Shrub, 1-2 m. high, branching copiously; flowers pink. Endemic. Plate 27, fig. 1.

\section{Clidemia D. Don.}

C. Neglecta D. Don, Mem. Wern. soc., vol. 4, p. 307 (1823); Mart., Fl. Bras., vol. 14, pt. 4, p. 483.- El Valle, alt. 600 m., Miller \& Johnston, no. 33, July 25. Further distribution, Peru and Brazil.

Miconia Ruiz \& Pav.

M. Laevigata (L.) DC., Prod., vol. 3, p. 188 (1S2S). Melastoma laevigata L., Sp. pl., ed. 2, vol. 1, p. 559 (1762).-El Valle, Miller \& Johnston, no. 119, July 24; San Juan Mt., alt. 600 m., Johnston, no. 52, July 11. Venezuela: Colonia Tovar, Fendler, no. 2262, 1856-57. Further distribution, tropical America.

M. prasina (Sw.) DC., Prod., vol. 3, p. 188 (182S). Melastoma prasina Sw., Fl. Ind. Occ., vol. 2, p. 777 (1S00).- Juan Griego trail, alt. 450 m., Johnston, no. 53, Aug. 12-15. A slender tree, about 7 m. high, with small white flowers. Further distribution, tropical America.

\section{ONAGRACEAE.}

\section{Jussiaea L.}

J. suffruticosa L., Sp. pl., vol. 1, p. $38 S$ (1753); Griseb., Fl. Brit. W. Ind., p. 273.- Juan Griego, Miller \& Johnston, no. 45, Aug. 9. Common to tropical countries.

\section{ARALIACEAE.}

\section{Gilibertia Ruiz \& Pav.}

G. ARborea (L.) March. in Durand \& Pittier, Bull. soc. bot. Belg., vol. 30, p. 281 (1S91). Aralia arborea L., Pl. Jam. Pugill., pt. 2, p. 11, and Syst. nat., ed. 10, vol. 2, P. 967 (1759). Dendropanax arboreum 
Dene. \& Planch., Rev. hort., ser. 4, vol. 3, p. 107 (1S54).- Juan Griego trail, alt. $450 \mathrm{~m}$., Johnston, no. 112, July 31. Further distribution, tropical America. A tree, $20 \mathrm{~m}$. high, with a clear trunk, which is $10 \mathrm{~m}$. high and $35 \mathrm{~cm}$. in diameter. Spread of foliage about $15 \mathrm{~m}$.

\section{Oreopanax Dene. \& Planch.}

O. Capitatum (Jacq.) Dene. \& Planch., Rev. hort., ser. 4, vol. 3, p. 108 (1S54). A ralia capitata Jacq., Hist. Stirp. Am., p. $S 9$ (1763).Juan Griego trail, alt. 450 m., Johnston, no. 308, Aug. 12-15. Venezuela: Colonia Tovar, Fendler, nos. 1320 and 526, 185t-55; Funk \& Schlim, no. 91, according to Seemann, Journ. bot., vol. 3, p. 270. Further distribution, Jamaica and Brazil.

\section{ERICACEAE.}

\section{VACCINIUII L.}

V. Latifolium B. \& H. f., Gen., vol. 2, p. 575 (1876), by implication. Thibaudia latifolia Griseb., Fl. Brit. W. Ind., p. 143 (1859).San Juan Mt., alt. 700 m., Johnston, no. 272, Aug. 28; also found on Juan Griego trail, alt. 450 m., July 31. Further distribution, Trinidad.

\section{PLUMBAGINACEAE.}

\section{Plumbago L.}

P. Capensis Thunb., Prod. pl. Cap., p. 33 (1794).- El Valle, in a garden, Miller \& Johnston, no. 117, July 29. Cultivated in Margarita from South Africa.

P. scandens L., Sp. pl., ed. 2, vol. 1, p. 215 (1762); Griseb., Fl. Brit. W. Ind., p. 390.- Santa Ana, Ernst; El Valle, Miller \& Johnston, no. 36, July 17. Venezuela: lower Orinoco, Rusby \& Squires, no. 68, 1896. 'Tropical America.

\section{SAPO'TACEAE.}

Achras L.

A. Zapota L., Sp. pl., vol. 2, p. 1190 (1753); Jacq., Hist. Stirp. Am., p. 57, pl. 61. Sapota Aehras Mill., Gard. (lict., ed. S, no. 1 (1768); Griseb., Fl. Brit. IV. Ind., p. 399.- El Valle, Miller \& Johnston, no. 
103. Cultivated in El Valle, Asuncion, and Tacarigua. Found in tropical countries.

\section{Bumelia Sw.}

B. cuneata Sw., Fl. Ind. Occ., vol. 1, p. 496 (1797); Griseb., Fi. Brit. IY. Ind., p. 401; Jacquinia petiolata Johnston, Proc. Amer. acad. arts and sci., vol. 40, p. 693 (1905).- Pt. Mosquito, east of Laguna Chica, Johnston, no. 273, Aug. 10, and Coche, Aug. 5. Distribution general in West Indies.

\section{OLEACEAE.}

\section{LINOCIERA Sw.}

L. Caribaea (Jacq.) Knobl., Bot. Centralbl., vol. 61, p. 87 (1895). Chionanthus caribaea Jacq., Coll., vol. 2, p. 110 (1788). Linociera compacta R. Br., Prod., p. 523 (1810); Griseb., Fl. Brit. IV. Ind., p. 405.- Juan Griego trail, alt. 350 m., Johnston, no. 136, July 2. Tree, $10 \mathrm{~m}$. high. Further distribution, tropical America.

\section{GENTIANACEAE.}

\section{Coltovbea Aubl.}

C. oensiflora Mart., Nov. gen. et sp., vol. 2, p. 111, pl. 185 (1826); Griseb., Fl. Brit. W. Ind., p. 423.- San Juan Mt., alt. 650 m., Johnston, no. 123, July 6. Further distribution, tropical America. Flowers white.

\section{APOCYNACEAE.}

\section{Echites P. Br.}

E. secundiflora A. DC. in DC., Prod., vol. 8, p. 457 (1844).San Juan MIt., alt. 750 m., Johnston, no. 137, July 11. Venezuela: Colonia Tovar, Fendler, nos. 1033, 1034, 1854-55. Further distribution, Mexico.

E. subsagitTata Ruiz \& Pav., Fl. Per., vol. 2, p. 19 (1799); Griseb., Fl. Brit. W. Ind., p. 413.- Santa Ana, Ernst. Further distribution, tropical America.

E. Umbellata Jacq., Enum. pl. Carib., p. 13 (1760), and Hist. Stirp. Am., p. 30; Griseb., Fl. Brit. IV. Ind., p. 414.- Santa Ana, Emst. Further distribution, West Indies. 


\section{Nerium L.}

N. Oleander L., Sp. pl., vol. 1, p. 209 (1753).- El Valle, Miller \& Johuston, no. 209, July 11. Cultivated for ornament in all warm countries.

\section{Plumeria L.}

P. Alba L., Sp. pl., vol. 1, p. 210 (1753); Griseb., Fl. Brit. W. Ind., p. 411.- Santa Ana, Ernst. Venezuela: near Cumaná and Caracas, HBK., Nor. gen. et sp., vol. 3, p. 230. Distributed from Cuba to the French Ids.

P. Caracasava Johnston, Contrib. U. S. nat. herb., vol. 12, p. $10 \mathrm{~S}$ (1908)._El Valle, Miller \& Johnston, no. 100, July 24. Venezuela: between Caracas and La Guaira, alt. 500 m., Fendler, no. 1026, Aug. 16, 1855; La Guaira, Robinson \& Lyon, July 13, 1900.

\section{RaUvolfia L.}

R. Lamarkil DC., Prod., vol. S, p. 337 (1844); Griseb., Fl. Brit. IV. Ind., p. 408. - El Valle, Miller \& Johnston, no. 43, July 5, and Johnston, no. 135, July 15. Further distribution, West Indies.

\section{Taberyaeniontana L.}

T. Amigdalifolia Jacq., Enum. pl. Carib., p. 14 (1760); Bot. reg., vol. 4, p. 338.- El Valle, Miller \& Johnston, no. 90, and Johnston, no. 68, July 1. Venezuela: Colonia Tovar, Fendler, no. 1029, Mar. 13, 185̄. Further distribution, tropical America. Varying from a tree about $\$$ meters high with a trunk 1 decimeter in diameter at its base to a diffuse shrub in form.

T. psychotrifolia HBK., Nov gen. et sp., rol. 3, p. 227 (181s); Griseb., Fl. Brit. W. Ind., p. 409.- Santa Ana, Ernst. Venezuela: Sacupana, Rusby \& Squires, no. S5, 1896. Further distribution, Trinidad.

\section{Thevetia Adans.}

T. Nereifolia Juss. ex Steud., Nom., ed. 2, vol. 2, p. 680 (1841); Griseb., Fl. Brit. W. Ind., p. 407; Mart., Fl. Bras., vol. 6, pt. 1, pl. 10. - Santa Ana, Ernst; El Valle, garden, Miller \& Johnston, no. 101, July 31. Distribution, tropical America. 


\section{ASCLEPIADACEAE.}

\section{Asclepias L.}

A. curassavica L., Sp. pl., vol. 1, p. 215 (1753); Griseb., Fl. Brit. W. Ind., p. 419; Desc., Ant., vol. 2, p. 191, pl. 116.-Santa Ana, Ernst; El Valle, Miller \& Johnston, no. S4, July 11. Venezuela: near Cumaná, HBK., Nov. gen. et sp., vol. 3, p. 190; Consejo, Fendler, no. 1037, Mar. 13, 1855; Sacupana, Rusby \& Squires, no. 26, April, 1896. Further distribution, tropical America.

\section{Calotropis R. Br.}

C. Procera (WVilld.) Dryand. in Ait. f., Hort. Kew., ed. 2, vol. 2, p. $7 S$ (1811); Griseb., Fl. Brit. W. Ind., p. 420; Bot. reg., vol. 21, pl. 1792. Asclepias procera Willd. Sp. pl., rol. 1, p. 1263 (1798).Juan Griego, Ernst; El Valle, Miller \& Johnston, no. 252, July 6. Venezuela: La Guaira, Fendler, no. 1053, Aug. 16, 1S55. Common in tropical countries.

\section{Ditassa R. Br.}

D. subulata Johnston, Proc. Amer. acad. arts and sci., vol. 40, p. 694 (1905).- San Juan Mt., climbing orer low shrubs at the summit, alt. 710 m., Johnston, no. 262, July 6 . Endemic.

\section{IBATIA Dene.}

I. maritima (L.) Dene. in DC., Prod., vol. S, p. 599 (18t4). Cynanchum maritimum L., Mant., p. $5+$ (1767); Jacq., Hist. Strip. Am., p. 83, pl.56. Ibatia muricata Griseb., Fl. Brit. W. Ind., p. 421 (1S61).Juan Griego, Emst. Further distribution, West Indies, Venezuela.

\section{Marsdenia R. Br.}

M. maculata Hook., Bot. mag., pl. 4299 (1S47); Griseb., Fl. Brit. W. Ind., p. 422.-El Valle, Miller \& Johnston, no. 17, July 18, and Johnston, no. 67, Aug. S. A rine hanging from trees. Further distribution, Ceutral America and Colombia.

\section{Metastelaia R. Br.}

M. Schlectendahlit Dene. in DC., Prod., vol. S, p. 513 (1S44); Griseb., Fl. Brit. W. Ind., p. 417.-El Valle, Miller \& Johnston, no. 14, July 14. Tenezuela: according to Emst in Seem., Journ. bot., vol. 5, p. 294 (1567). Further distribution, tropical America. 
Sarcostemala R. Br.

S. GLAUCA HBK., Nov. gen. et sp., vol. 3, p. 194, pl. 229 (181S). - Santa Ana, Ernst. Venezuela: near La Guaira, Maiqueti, and Cabo Blanco, HBK., l. $c$.

\section{CONVOLVULACEAE.}

\section{Cuscuta L.}

C. australis R. Br., Prod., p. 491 (1810). C. obtusiflora HBK., Nor. gen. et sp., rol. 3, p. 122 (1S1S); Mart., Fl. Bras., vol. 7, p. 3S0, pl. 127; Griseb., Fl. Brit. W. Ind., p. 476.-Santa Ana, Ernst. Distributed from Florida to Pert.

\section{Evolvulus L.}

E. Arexicola Johnston, Proc. Amer. acad. arts and sci., vol. t0, p. 694 (1905).- Along the trail from Porlamar to San Antonio, Miller \&. Johnston, no. 21S, Aug. 2. Endemic.

E. Filipes Mart., Flora, rol. 24, pt. 2, Beibl., p. 100 (1841).- El Valle, Miller \& Johnston, no. 16, Aug. 9. Further distribution, Brazil.

E. Incanes Pers., Syn., vol. 1, p. 288 (1805). E. sericeus Sw., Prod. reg. Ind. Occ., p. 55 (17SS); Griseb., Fl. Brit. W. Ind., p. 475. - Santa Ana, Emst. Distribution general in tropical America.

E. mucronatus Sw. ex Wikstr., Vet. akad. handl. Stockh., p. 61 (1827); Griseb., Fl. Brit. W. Ind., p. 475.- Santa Ana, Emst. Distributed from Porto Rico to Peru.

\section{Iponioea L.}

I. Batatas Poir., Encycl., vol. 6, p. 14 (1S04); Griseb., Fl. Brit. W. Ind., p. 46S. Cultivated, according to Ernst. Commonly cultivated in warm climates.

I. PES-CAPrae (L.) Sweet, Hort. suburb. Lond., p. 35 (1S18); Griseb., Fl. Brit. W. Ind., p. 470. Convolvulus pes caprae L., Sp. pl., rol. 1, p. 159 (1753). I pomoea biloba Forsk., Fl. Aegypt.-Arab., p. 44 (1775).- Juan Griego, Ernst. Venezuela: Colonia Torar, Fendler, no. 937, 1S51-55. All tropical countries.

I. CARNed Jacq., Enum. pl. Carib., p. 13 (1760); Griseb., Fl. Brit. 
W. Ind., p. 469.- El Valle, Miller \& Johnston, no. 79, July 18, and Johnston, no. S0, Aug. S, and no. 39. Further distribution, Nicaragua, Jamaica, Colombia.

I. Coccinea L., Sp. pl., vol. 1, p. 160 (1753); Griseb., Fl. Britt. W. Ind., p. 472.-El Valle, Miller \& Johnston, no. 75, July 19. Venezucla: Colonia Tovar, Fendler, nos. 933, 2075, 1S54-57. Further distribution, temperate and tropical countries.

I. Quanoclit L., Sp. pl., vol. 1, p. 159 (1753); Griseb., Fl. Brit. IV. Ind., p. 472.- El Valle, Miller \& Johnston, no. 76, July 12. Venezuela: near Cumanacoa and Angostura, HBK., Nov. gen. et sp., vol. 3, p. 110. Further distribution, tropical countries.

I. Sivuata Orteg., Hort. Matr. dec., S4 (179S). I. dissecta Pursh ace. to Griseb., Fl. Brit. W. Ind., p. 467.-Asuncion, Willer \& Johnston, no. 78, Aug. 9. Venezuela: Ernst in Seem., Journ. bot., 1S67, p. 290-296. Further distribution, tropical countries.

I. Trifid A. Don, Gen. syst., vol. 4, p. 280 (1S38).-El Valle, Miller \& Johnston, no. 77, July 16. Venezuela: La Victoria, Fendler, no. $207+$ B, Nov. 21, 1S56. Further distribution, tropical America.

I. tuberosa L., Sp. pl., vol. 1, p. 160 (1753); Griseb., Fl. Brit. IV. Ind., p. 467.- Santa Ana, Emst. Further distribution, tropical countries.

\section{JACQUEMONTIA Choisy.}

J. violaces (Vahl.) Choisy in Mém. soc. phỵs. Genèr., vol. S, p. 61 (1S38). Convolvulus violaceus Vahl, Symb., vol. 3, p. 29 (1794). C. pentanthos Jacq., Coll., vol. 4, p. 210 (1790); Griseb., Fl. Brit. IW. Ind., p. 474.-El Valle, Miller \& Johnston, no. 80, July 12. Venezuela: Ernst in Seem., Journ. bot., 1867, p. 293. Further distribution, Cuba and Mexico to Peru.

\section{BORRAGINACEAE.}

\section{Bourreria P. Br.}

B. exsccca Jacq., Enum. pl. Carib., p. 14 (1760); Griseb., Fl. Brit. W. Ind., p. 482.-- Santa Ana, Ernst; El Valle, Miller \& Johnston, no. 10, July S, and Johnston, no. S6, July 15. A slender tree, 3 to $4 \mathrm{~m}$. high. Further distribution, St. Vincent, Colombia, Venezuela.

\section{Cordia I.}

C. Alba (Jacq.) Roem. \& Schult., Syst., vol. 4, p. 466 (1S19); Griseb., Fl. Brit. W. Ind., p. 478. Tarronia alba Jacq., Hist. Stirp. 
Am., p. 41 (1763).- El Valle, Miller \& Johnston, no. 72, July 6, and Johnston, no. 57, July 24. Venezuela: Ernst, Sobre la flora y fauna, p. 227; La Guaira, Fendler, no. 921, Aug. 16, 1S5.5. A slender tree, 5 m. high. Further distribution, tropical America.

C. cylindristachya (Ruiz \& Pav.) Roem. \& Schult., Syst., vol. 4, p. 459 (1819); Griseb., Fl. Brit. W. Ind., p. 480. V'arronia cylindrostachya Ruiz \& Pav., Fl. Peruv., vol. 2, p. 23, pl. 147 (1799). - Juan Griego, Santa Ana, Emst; El Valle, Miller \& Johnston, no. 26, July 4, and Johnston, no. 85, July 15. Further distribution, Lower California, W'est Indies to Uruguay.

C. Discolor Cham., Linnaea, vol. 4, p. 4S2 (1S29) - San Juan MIt., alt. $570 \mathrm{~m}$., Johnston, no. 296, July 19 . Leaves narrower than in the typical specimen. Further distribution, Brazil and Bolivia.

C. Gerascanthus Jaeq., Hist. Stirp. Am., p. 43, pl. 175 (1763); Griseb., Fl. Brit. W. Ind., p. 478. - Santa Ana, Emst. Venezuela: Colonia 'Tovar, Fendler, no. 2054, 1850-57. Further distribution, West Indies, Guatemala to Brazil.

C. GLobosa (Jacq.) HBK., Nov. gen. et sp., vol. 3, p. 76 (1S1S); Griseb., Fl. Brit. W. Ind., p. 481. Varronia globosa Jacq., Enum. pl. Carib., p. 14 (1760).-- El Valle, Miller \& Johnston, no. 221, July 1S; Asuncion, Johnston, no. S4, July 9. Slender bush, 2-3 in. high. Venezuela: Ernst, Sobre la flora y fauna, p. 227. Further distribution, tropical America.

C. Reticulata Vahl, Eelog. Am., vol. 3, p. 5 (1S07); Griseb., Fl. Brit. W. Ind., p. 479.- Juan Griego, Ernst. Further distribution, West Indies, Venezuela.

C. Sebestena L., Sp. pl., vol. 1, p. 190 (1753); Griseb., Fl. Brit. IV. Ind., p. 478; Jacq., Hist. Stirp. Am., p. 42.-Juan Griego, Emst; El Valle, Miller \& Johnston, no. 219, July 26. Further distribution, West Indies to Guiana and Colombia.

\section{Heliotropium L.}

H. curassavicum L., Sp. pl., vol. 1, p. 130 (1753); Griseb., Fl. Brit. W. Ind., p. 486.- Juan Griego, Ernst. Venezuela: Tortuga, Ernst in Seem., Journ. bot., vol. 14, p. 179 (1876). Common to tropical countries.

H. Filiforue HBK., Nov. gen. et sp., vol. 3, p. S6, pl. 204 (1S18); Griseb., Fl. Brit. W. Ind., p. 486.- El Valle, Miller \& Johnston, no. 14S, Aug. 8; Juan Griego, Johnston, no. 331, Aug. 14. Venezuela: at Rio Apure, between the towns of El Diamente and San Fernando, 
HBK., Nor. gen. et sp., vol. 3, p. 86 . Further distribution, American tropies.

H. Inideur L., Sp. pl., vol. 1, p. 130 (1753); Griseb., Fl. Brit. IV. Ind., p. 485.- Juan Griego, Santa Ana, Asuncion, Ernst; El Valle, Miller \& Johnston, no. 206, July 5, and Johnston, no. 49, July 15. Found in all tropical countries.

H. Parviflorum L., Mant., vol. 2, p. 201 (1771); Griseb., Fl. Brit. W. Ind., p. 485.-El Valle, Miller \& Johnston, no. 32, July 7, and Johnston, no. 93, Aug. S; also found at Juan Griego. Further distribution, tropical America.

\section{Tournefortia L.}

T. GNaphalodes R. Br., Prod., p. 496 (1810), by implication; Griseb., Fl. Brit. Wr. Ind., p. 483.- Juan Griego, Emst.; Pt. Moreno, Johnston, no. 105, July S. Further distribution, Florida, Bahamas, West Indies. A shrub, 0.5-1 m. high, growing in the loose sand near the sea.

T. Hirsutissima L., Sp. pl., vol. 1, p. 140 (1753); Griseb., Fl. Brit. W. Ind., p. 483.-El Valle, Miller \& Johnston, no. 47. Venezuela: near Cumaná and Bordones, HBK., Nov. gen. et sp., rol. 3, p. 80. Further distribution, tropical America.

T. Incana Lam., Illust., vol. 1, p. 417 (1791); Griseb., Fl. Brit. IV. Ind., p. 485.- Santa Ana, Enst. Further distribution, Cuba, Haiti.

T. scandens Mill., Gard. dict., ed. S, no. 4 (1768).-El Valle, Miller \&. Jolinston, no. 2, Aug. 2, and no. 249, July 20, and Johnston, no. 254, July 27. Further distribution, Jamaica.

T'. volubilis L., Sp. pl., rol. 1, p. 140 (1753); Griseb., Fl. Brit. IV. Ind., p. 4St.- Santa Ana, Ernst. Further distribution, tropical America.

T. sp. Shrubby; leaves oral, entire, glabrous on upper side, pilose on lower side, with acuminate apex and rounded unequal base, $4 \mathrm{~cm}$. wide and $\mathrm{Sem}$. long, and smaller; petiole 5 to $10 \mathrm{~mm}$. long: inflorescence cymose, reddish-pubescent; (alyx-lobes narrowly acute.-El Valle to San Juan, Johnston, no. \&3, July 11.

\section{VERBENACEAE.}

\section{Avicennia L.}

A. NitidA Jacq., Enum. pl. Carib., p. 25 (1760), and Jacq., Hist. Stirp. Am., p. 177.- Pt. Mosquito, Johnston, no. 139, Aug. 10. 
Venezuela: Ernst, Sobre la flora y fauna, p. 223. Further distribution, Cuba to Brazil, tropical Africa.

\section{Bocchea Cham.}

B. Ehrenbergir Cham. in Linnaea, vol. 7, p. 253 (1832); Griseb., Fl. Brit. W. Ind., p. 493.- El Valle, Miller \& Johnston, no. 205, July 20. Venezuela: La Guaira according to Schaner, Linnaea, vol. 20, p. 478. Further distribution, tropical America.

\section{Citharexylum Mill.}

C. quadrangulare Jacq., Enum. pl. Carib., p. 26 (1760); Griseb., Fl. Brit. W. Ind., p. 497; Jacq., Hort. Vind., vol. 1, pl. 22.-Santa Ana, Ernst. Further distribution, West Indies, Guiana.

\section{Clemodendrox L.}

C. Molde HBK., Nor. gen. et sp., rol. 2, p. 244 (1817).- El Valle, Miller \& Johnston, no. S, July 30; en route El Valle to Asuncion, Johnston, no. 82, July 9. A form with small flowers, especially a small calyx. Short spines occur in some axils. Further distribution, Peru and Galapagos Ids.

\section{Duranta L.}

D. Plumieri Jacq., Hist. Stirp. Am., p. 156, pl. 176, fig. 76 (1763); Griseb., Fl. Brit. W. Ind., p. 49S.-Santa Ana, Ernst; El Valle, Miller \& Johnston, no. 115, July 15. Venezuela: Cumaná, Moritz, no. 424, according to Schaner, Limnaea, vol. 20, p. 482. Further distribution, tropical America.

LANTANA L.

L. Camara L., Sp. pl., vol. 2, p. 627 (1753); Griseb., Fl. Brit. IV. Ind., p. 495; Desc., Ant., vol. 4, p. 330, pl. 304.-Santa Ana, Ernst; El Valle, Miller \& Johnston, no. 95, July 6. Venezuela: Caracas and Valencia, Moritz, according to Schauer, Linnaea, rol. 20, p. 481; Colonia Tovar, Fendler, no. 860 (in part), Jan. 31, Feb. 23, 1854; Sacupana, Rusby \& Squires, no. 4; Paloma, Rusby \& Squires, no. 312; Caracas, A. II. Moore, Mar. 16, 1899; San Julian, Robinson \& Lyon, July 17, 1900. Distribution general in tropical America.

L. Lilacina Desf., Cat. hort. Par., ed. 3, p. 392 (1S29); Schauer in Mart., Fl. Bras., vol. 9, p. 261, pl. 44, fig. 1. L. stricta, var. lilacina 
Griseb., Fl. Brit. W. Ind., p. 496 (1861).—El Valle, Miller \& Johnston, no. 94. Venezuela: Caracas, Moritz, according to Schauer, Linnaea, vol. 20, p. 481. Distributed from Mexico to Brazil.

L. Reticulata Pers., Syn., vol. 2, p. 141 (1807); Griseb., Fl. Brit. W. Incl., p. 496; Ernst, Bot. Excurs. Margarita, p. 7. L. Sellowiana Link \& Otto, Ic. pl. sel. Berol., p. 107, pl. 50 (1S28).-- Santa Ana, Ernst. Further distribution, West Indies, Venezuela.

L. trifolia L., Sp. pl., vol. 2, p. 626 (1753); Griseb., Fl. Brit. IV. Ind., p. 496.- Santa Ana, Ernst. Venezuela: Aragua, E. Otto \& Moritz, according to Schauer, Linnaea, vol. 20, p. 4S1. Further distribution, tropical America.

\section{LIPPIA L.}

L. GEminata HBK., Nov. gen. et sp., vol. 2, p. 266 (1817); Griseb., Fl. Brit. IV. Ind., p. 495.-Santa Ana, Ernst; El Valle, Viller \& Johnston, no. 125, Aug. 16, and Johnston, no. 81, Aug. S. Further distribution, tropical America. A spreading bush, $1 \mathrm{~m}$. high.

L. meronera Schauer in DC., Prod., vol. 11, p. 587 (1847); Griseb., Fl. Brit. WT. Ind., p. 495.- Juan Griego, Ernst. Venezuela: Angostura, Moritz, according to Schauer, Linnaea, vol. 20, p. 480. Distributed from West Indies to Guiana.

\section{Priva Adans.}

P. Lappulacea (L.) Pers., Syn., vol. 2, p. 139 (1807). P. echinata Juss., Ann. mus. Par., vol. 7, p. 69 (1806); Griseb., Fl. Brit. IV. Ind., p. 493. Verbena lappulacea L., Sp. pl., vol. 1, p. 19 (1753).- Santa Ana, Emst; El Valle, Miller \& Johnston, no. 96, July 9. Further distribution, tropical America.

\section{Stachytarpheta Vahl.}

S. Cajanexsis Vahl, Enum., vol. 1, p. 208 (1804); Griseb., Fl. Brit. IV. Ind., p. 494.- Sinta Ana, Ernst; El Valle, Miller \& Johnston, no. 93, July 6, and Johnston, no. 92, Aug. S. Venezuela: near Cumaná, Bordones, and Caripe, HBK., Nov. gen. et sp., vol. 2, p. 280; Guayre River, Moritz, and Orinoco, S. Ayres, according to Schauer, Linnaea, vol. 20, p. 47s. Further distribution, tropical America.

S. Jaldicexsis (L.) Tahl, Enum., vol. 1, p. 206 (1804); Griseb., Fl. Brit. W. Ind., p. 494. Verbena jamaicensis L., Sp. pl., vol. 1, p. 19 (1753). S. indica Vahl, Enum., vol. 1, p. 206 (1S04); Griseb., 
Fl. Brit. W. Ind., p. 494. Verbena indica L., Sp. pl., ed. 2, vol. 1, p. 27 (1762).-EI Valle, Miller \& Johnston, no.92, July 5. Venezuela: near Cumaná, HBK., Nov. gen. et sp., rol. 2, p. 279. Further distribution, tropical countries.

\section{LABIATAE. \\ Coleus Lour.}

C. Amboinicus Lour., Fl. Cochinch., vol. 2, p. 372 (1790); Griseb., Fl. Brit. IV. Ind., p. 487. Plectranthus aromaticus Roxb., Hort. Bengal., p. 45 (1S14). C. aromaticus Benth. in Wallich, Pl. As. rar., vol. 2, p. 15 (1831); Bot. reg., vol. 18, pl. 1520.- Santa Ana, Emst. Introduced into the West Indies from the East Indies.

\section{Hyptis Jacq.}

H. Capitata Jacq., Coll., vol. 1, p. 102 (1786), and Ic. rar., vol. 1, pl. 114; Griseb., Fl. Brit. W. Ind., p. 4SS.-Santa Ana, Ernst. Further distribution, tropical America, Manila.

H. Pectinata (L.) Poit., Ann. mus. Par., vol. 7, p. 474, pl. 30 (1806); Griseb., Fl. Brit. W. Ind., p. 489. Nepeta pectinata L.. Syst. nat., ed. 10, vol. 2, p. 1097 (1759).- El Valle, Miller \& Johnston, no. 110, July 24. Further distribution, tropical America, East Indies.

LeONotis R. Br.

L. Nepetifolia R. Br. in Ait. f., Hort. Kew., ed. 2, vol. 3, p. 409 (1S11); Griseb., Fl. Brit. W. Ind., p. 492.-Santa Ana, Ernst; El Valle, Miller \& Johnston, no. 74, July 19. Common in tropical countries.

\section{Salvia L.}

S. coccinea Juss. ex Murr., Comm. Goetting., vol. 1, p. 86, pl. 1 (1778); Griseb., Fl. Brit. W. Ind., p. 490.-Santa Ana, Ernst. Further distribution, tropical America.

S. occidentalis Sw., Prod. veg. Ind. Occ., p. 14 (1788); Griseb., Fl. Brit. W. Ind., p. 490.- Santa Ana, Ernst. Further distribution, tropical America.

S. tiliaefolia Tahl, Symb. bot., vol. 3, p. 7 (1794).- Santa Ana, Ernst. Venezuela: valley of Caracas, HBK., Nov. gen. et sp., vol. 2, p. 299 (as S. fimbriata). Further distribution, Mexico. 


\section{SOLANACEAE.}

Bassovia Aubl.

B. Ciliata Johnston, Proc. Amer. acad. arts and sci., vol. 40, p. $69+$ (1905). - El Valle, River trail, Miller \& Johnston, no. 255, July 15, and Johnston, no. 75, Aug. 30.

\section{Brachistus Miers.}

B. Pringlei Wats., Proc. Amer. acad. arts and sci., vol. 25, p. 159 (1890).- El Valle, Miller \& Johnston, no. 35, July 27. Further distribution, Mexico, Central America, and Colombia.

\section{Brunfelsia I.}

B. Hopeana (Hook.) Benth. in DC., Prod., vol. 10, p. 200 (1846); Griseb., Fl. Brit. W. Ind., p. 432; Mart., Fl. Bras., vol. S, pt. 1, p. 261, pl. 43. Francisera Hopeana Hook., Bot. mag., pl. 2829 (1S2S).El Valle, Miller \& Johnston, no. 265, Aug. 2, and Johnston, no. 71, Aug. 31 on South Hill. Further distribution, Trinidad, Brazil.

\section{Capsicum L.}

C. Annuum L., Sp. pl., vol. 1, p. $18 S$ (1753); Desc., Ant., vol. 6, p. 172, pl. 422.-- El Valle, Miller \& Johnston, no. \$1, July 24. Venezuela: Cumaná, Loefling, Reise nach den spanischen Ländern, p. 152153. All tropical countries.

C. Baccatum L., Mant., vol. 1, p. 47 (1767); Griseb., Fl. Brit. IV. Ind., p. 436; Desc., Ant., vol. 6, p. 177, pl. 423.-El Valle, Miller \& Johnston, no. S2, July 12 . Venezuela: between Cumaná and Bordones, HBK., Nov. gen. et sp., vol. 3, p. 49. Found in all tropical countries.

C. frutescens L., Sp. pl., vol. 1, p. 189 (1753); Griseb., Fl. Brit. W. Ind., p. 436; Lam., Encycl., pl. 116.-Cultivated, according to Ernst. Venezuela: near Bordones, HBK., Nov. gen. et sp., vol. 3, p. 48. Found in all tropical countries.

\section{Cestrum L.}

C. vespertinum L., Mant., vol. 2, p. 206 (1771); Griseb., Fl. Brit. IV. Ind., p. 443; Jacq., Hort. Schoenbr., vol. 3, pl. 32S.- River trail, El Valle, Johnston, no. 285, Aug. 30. Further distribution, West Indies to Panama, Peru, and Brazil. 


\section{Datura L.}

D. Metel L., Sp. pl., vol. 1, p. 179 (1753); Griseb., Fl. Brit. W. Ind., p. 434; Bot. mag., vol. 35, pl. 1440.-El Valle, Miller \& Johnston, no. \$3, July 30. Further distribution, tropical America, tropical Africa, Spain.

D. Tatula L., Sp. pl., ed. 2, vol. 1, p. 256 (1762); Griseb., Fl. Brit. W. Ind., p. 434; Sweet, Brit. fl. gard., vol. 1, pl. S3.-Santa Ana, Ernst. Further distribution, tropical and temperate countries.

\section{LYCIUMI L.}

L. SAlsum Ruiz \& Pav., Fl. Per., vol. 2, p. 46, pl. 183 (1799).El Valle, Miller \& Johnston, no. 153, July 31, and Johnston, no. 61, Aug. 3. Further distribution, Peru.

\section{LrCOPERSICUM Hill.}

L. Humboldtir (Willd.) Dunal, Solan., p. 112 (1813); Griseb., Fl. Brit. W. Ind., p. 436. Solanum IIumboldtii Willd., Hort. Berol., p. 27, pl. 27 (1804).-Cultivated, according to Ernst. Distributed from Mexico to Brazil.

\section{Nicotiana L.}

N. Tabacum L., Sp. pl., vol. 1, p. 180 (1753); Griseb., Fl. Brit. IT. Ind., p. 434.- Cultivated. Widely grown in temperate and tropical countries.

\section{Phisalis L.}

P. Peruviana L., Sp. pl., ed. 2, vol. 2, p. 1670 (1763); Griseb., Fl. Brit. W. Ind., p. 435.- Santa Ana, Ernst. Widely distributed in temperate and tropical countries.

\section{Solanum L.}

S. aculeatissinum Jacq., Coll., vol. 1, p. 100 (17S6); Griseb., Fl. Brit. IV. Ind., p. 442.- Santa Ana, Ernst. Further distribution, tropical America.

S. Hirtum Vahl, Symb. bot., vol. 2, p. 40 (1791).- Santa Ana Ernst.

S. Lanceaffolium Jacq., Coll., vol. 2, p. 286 (17SS).- San Juan Mt., alt. $790 \mathrm{~m}$., Johnston, no. 69, July 6. Slender shrub, ascending, 1-1.5 m. high. Further distribution, West Indies, Venezuela. 
S. ifargaritense Johnston, Proc. Amer. acad. arts and sci., vol. 40, p. 695 (1905). - El Valle, a single ascending stem branching copiously near the top, found at the side of the River trail, Johnston, no. 315, Aug. 20. Plate 29, fig. 2.

S. Nigrum L., Sp. pl., vol. 1, p. 186 (1753). S. nodiflorum Jacq., Ic. pl. rar., vol. 2, p. 11, pl. 326 (1781); Griseb., Fl. Brit. IV. Ind., p. 437.- Santa Ana, Ernst; El Valle, Johnston, no. 73, July 16. Venezuela: Cumaná, Loefling, Reise nach den spanischen Ländern, p. 152-153. A low herb, about $5 \mathrm{dm}$. high. Temperate and tropical countries.

S. Polyganum, Vahl, Symb. bot., vol. 3, p. 39, pl. 55 (1794); Griseb., Fl. Brit. W. Ind., p. 442.- El Valle, Miller \& Johnston, no. 143, July 18. Further distribution, Trinidad, St. 'Thomas, St. Croix.

S. Seaforthinanui Andr., Bot. rep., vol. 8, pl. 504 (1799-1811). - El Valle, Miller \& Johnston, no. 104, July 18. Further distribution, tropical America.

S. Triste, Jacq., Enum. pl. Carib., p. 15 (1760); Griseb., Fl. Brit. W. Ind., p. 437.- Juan Griego trail, alt. 450 m., Johnston, no. 70, July 22. Venezuela: near Cumanacoa and Guanaguana, HBK., Nov. gen. et sp., vol. 3, p. 34. Further distribution, tropical America.

S. verbascifolium L., Sp. pl., vol. 1, p. 184 (1753); Griseb., Fl. Brit. W. Ind., p. 438. - Santa Ana, Ernst; El Valle, Miller \& Johnston, no. 22:2, and Johnston, no. 74, July 15. Venezuela: near Cumaná, HBK., Nor. gen. et sp., vol. 3, p. 30; Ernst in Seemann's Journ. bot., vol. 3, p. 319. Further distribution, tropical America to East Indies. A low shrub, about $1 \mathrm{~m}$. high.

S. umbritile Johnston, Proc. Amer. acad. arts and sci., vol. 40, p. 695 (1905).- Rio Asuncion, in the heavy woods along the trail to Juan Griego, Johnston, no. 321, July 22.

\section{SCROPHULARIACEAE.}

Berrichia Cham. \& Schlecht.

B. scltellarioides Benth., Scroph. Ind., p. 9 (1835), in note; Griseb., Fl. Brit. W. Ind., p. 429; Mart., Fl. Bras., vol. 8, pt. 1, pl. 50, fig. 2.- Santa Ana, Emst. Further distribution, Trinidad, Venezuela, Brazil.

\section{Capraria L.}

C. BIflora L., Sp. pl., vol. 2, p. 628 (1753); Griseb., Fl. Brit. W. Ind., p. 427; Desc., Ant., vol. 4, p. 313, pl. 300.- Santa Ana, Ernst, 
reported as C. mexicana; El Valle, Miller \& Johnston, no. 41, July 30, and Johnston, no. 265, Aug. S. Venezuela: near Cumaná, HBK., Nor. gen. et sp., vol. 2, p. 354. Further distribution, tropical America and tropical Africa.

\section{ILYSANTHES Rafin.}

I. Riparia Rafin., Ann. nat., p. 13 (1820).- Juan Griego, Johnston, no. 125, Aug. 14. Further distribution, tropical America.

\section{SCOPARIA L.}

S. Dulcis L., Sp. pl., vol. 1, p. 116 (1753); Griseb., Fl. Brit. W. Ind., p. 427.- Juan Griego, Ernst; El Valle, Miller \& Johnston, no. 144, Aug. 11. Venezuela: between Caracas and Bordones, HBK., Nor. gen. et sp., rol. 2, p. 354; Cumaná, Loefling, Reise nach den spanischen Iändern, p. 152-153. Further distribution, tropical countries.

\section{BIGNONIACEAE.}

\section{Bignonia L.}

B. Acuminata Johnston, Proc. Amer. acad. arts and sci., vol. 40, p. 696 (1905).- El Valle, Johnston, no. 345. Endemic. Plate 29, figs. $1,1 \mathrm{a}, 1 \mathrm{~b}$.

B. AEquinoctialis L., Sp. pl., vol. 2, p. 623 (1753); Griseb., Fl. Brit. W. Ind., p. 448; Plum., Ic. Burm., pl. 55.-El Valle, Johnston, no. 78 , Aug. S. Further distribution, tropical America. A rine or sprawling shrub.

B. sP.- El Valle, Miller \& Johnston, no. 154, Aug. 6. Leaves trifoliolate, glabrous, smooth; calyx cupulate; margin undulate, shortly 5-parted; corolla purple, 5-lobed, slightly 2-lipped, about $2.5 \mathrm{~cm}$. long.

B. sp.-El Valle, Johnston, no. 77, Aug. 15. Calyx cupulate, with 5 procurrent short teeth; corolla purple, 5-lobed, slightly 2lipped, $3-5 \mathrm{~cm}$. long.

\section{Crescentia L.}

C. Cujete L., Sp. pl., vol. 2, p. 626 (1753); Jacq., Hist. Stirp. Am., p. 175; Griseb., Fl. Brit. IV. Ind., p. 445.- Santa Ana, Ernst; El Valle, Johnston, no. 248. A tree, about $4 \mathrm{~m}$. high, with widespreading top and trunk $2.5 \mathrm{dm}$. in diameter at the base. Common to tropical America. 


\section{MaCfadyena A. DC.}

M. Cormibosa Griseb., Bonplandia, vol. 6, p. 10 (1858).-El Vallc, Johnston, no. 255, July 24. Distributed from Panama to equatorial Brazil.

\section{Tabebuia Gom.}

T. Rufescens Jolnston, Proc. Amer. acad. arts and sci., vol. 40, p. 696 (1905).--San Juan MIt, alt. 500 m., Johnston, no. 79, July 2. Endemic.

\section{Teconta Juss.}

T. stans (L.) Juss., Gen., p. 139 (1789); Griseb., Fl. Brit. IV. Ind., p. 447. Bignonia stans L., Sp. pl., ed. 2, vol. 2, p. $S 71$ (1762).-El Valle, Willer \& Johnston, no. 220, July 1S, and Johnston, no. 76, July 24. Venezuela: MIt. Cocollar, HBK., Nov. gen. et sp., vol. 3, p. 144. Further distribution, Mexico and West Indies.

\section{MAR'TYNIACEAE.}

\section{Craniolaria L.}

C. AnvuA L., Sp. pl., vol. 2, p. 61 (1753); Jacq., Hist. Stirp. Am., p. 173; Griseb., Fl. Brit. WV. Ind., p. 466.-El Valle, Miller \& Johnston, no. 89, July 12. Venezuela: Ernst, Sobre la flora y fauna, p. 227; llanos of New Barcelona, HBK., Nov. gen. et sp., vol. 3, p. 153. Distribution, New Mexico to Tenezuela.

\section{GESNERIACEAE.}

\section{Drymonia Mart.}

D. serrulata (Jacq.) Mart., Nor. gen. et sp., vol. 3, p. 59 (1S29). D. bicolor Lindl., Bot. reg., vol. 24. pl. 4 (1S38). Besleria serrulata Jacq., Hort. Schoenb., vol. 3, p. 21 (179S).- Rio Asuncion, alt. 400500 m., Johnston, 110. 27S, Aug. 12-15. Found in the West Indies.

\section{LENTIBULARIACEAE.}

\section{Utricularia I.}

U. alpina Jacq., Enum. pl. Carib., p. 11 (1760). U. montana Jacq., Hist. Stirp. Am., p. 7, pl. 6 (1763); Griseb., Fl. Brit. IV. Ind., 
p. 390.- San Juan Mt., alt. 600 m., Miller \& Johnston, no. 271, Aug. 2, and Johnston, no. 223, July 2. Further distribution, tropical America.

\section{ACAN'THACEAE.}

\section{DlaNTHERA L.}

D. SEcunda (Vahil) Griseb., Goett. Abh., vol. 7, p. 246 (1S57). Justicia secunda Vahl, Symb. bot., vol. 2, p. 7 (1791).- San Juan Mt., alt. $795 \mathrm{~m}$., Johnston, no. 89, July 2. Further distribution, tropical America.

\section{JACOBINIA Moric.}

J. PAdCiflora (Nees) Benth. \& Hook. f., Gen., vol. 2, p. 1115 (1S76). Sericographis pauciflora Nees in Mart., Fl. Bras., vol. 9, p. 110 (1847).- South Hill, El Valle, alt. 300 m., Johnston, no. 19, July 27. Further distribution, Brazil.

\section{Ruellia L.}

R. tuberosa L., Sp. pl., vol. 1, p. 635 (1753); Griseb., Fl. Brit. IV. Ind., p. 452.- El Talle, Willer \& Johnston, no. SS, July 12. Venezuela: Colonia Tovar, Fendler, no. 799, Aug. 16, 155.5. Further distribution, tropical America.

\section{Siphonoglossa Oerst.}

S. Pilosella (Nees) Torr., Bot. Mex. bound., p. 124 (1859). Monechma Pilosella Nees in DC., Prod., vol. 11, p. 412 (1S47).-- El Valle, Miller \& Johnston, no. 227, July 26. Further distribution, Texas, Mexico, and St. Thomas.

\section{RUBIACEAE.}

BasanaCANTHA Hook. f.

B. tetracantha (Cav.) Hook. f. in Benth. \& Hook. f., Gell., vol. 2, p. $\$ 3$ (1S73). Mussaenda tetracantha Cav., Ic., vol. 5, p. 20, pl. 435 (1799).- Santa Ana, Emst. American tropies.

\section{Cephaelis Sw.}

C. Iuscosa (Jacq.) Sw., Prod. reg. Ind. Occ., p. 46 (178s); Griseb., Fl. Brit. W. Ind., p. 347. Morinda muscosa Jacq., Hist. Stirp. Am., 
p. 65 , pl. 45 (1763).--San Juan Mt., alt. 500 m., Johnston, no. 113, July 11. Further distribution, West Indies, Guiana to Brazil. A slender shrub, 0.5-1 m. ligh.

\section{Chiococca P. Br.}

C. micrantha Johnston, Proc. Amer. acad. arts and sci., vol. 40, p. 696 (1905).- San Juan MIt., in woods above South Hill, alt. 400 m., Johnston, no. 115, July 27. Endemic. Plate 29, figs. 3, 3a, $3 \mathrm{~b}, 3 \mathrm{c}$.

C. Raceirosa Jacq., Hist. Stip. Am., p. 68 (1763); Griseb., Fl. Brit. WV. Ind., p. 336. - El Valle, Miller \& Johnston, no. 42, July 15, on bank of the River trail; Tacarigua, Johnston, no. 119, Aug. 15. Widely distributed in tropical America.

\section{Chomelia Jacq.}

C. spinosa Jacq., Enum. pl. Carib., p. 12 (1760), \& Hist. Stirp. Am., p. 18.- Santa Ana, Emst.

\section{Coutarea Aubl.}

C. hexandra (Jacq.), n. comb. Portlandia hexandra Jacq., Hist. Stirp. Am., p. 63, pl. 1S2, fig. 20 (1763). Coutarea speciosa Aubl., Pl. Guian., vol. 1, p. 31t, pl. 122 (1775); Griseb., Fl. Brit. IV. Ind., p. 323.- Santa Ana, Emst. Widely distributed in tropical America.

\section{Diodia L.}

D. RIGIDA (HBK.) Cham. \& Schlecht., Linnaea, vol. 3, p. 341 (182S); Griseb., Fl. Brit. W. Ind., p. 348. Spermacoce rigida HBK., Nov. gen. et sp., vol. 3, p. 342 (1S1S).- Santa Ana, Ernst. Further distribution, West Indies to Paraguay.

\section{Erithalis P. Br.}

E. Fruticosa L., Syrst. nat., ed. 10, vol. 2, p. 930 (1759); Griseb. Fl. Brit. W. Ind., p. 336.- Juan Griego, Ernst. Further distribution, tropical America.

Gonzalagunia Ruiz \& Par.

G. Hirstta (Jacq.) Schum. in Mart., Fl. Bras., vol. 6, pt. 6, p. 291 (1S59). Justicia hirsuta Jacq., Enum. pl. Carib., p. 11 (1760). 
Gonzalea spicata DC., Prod., rol. 4, p. 437 (1S30); Griseb., Fl. Brit; W. Ind., p. 321.-El Valle, Miller \& Johnston, no. 217, July 24. San Juan Mt., Johnston, no. 100, July 11. Distribution general in tropical America.

\section{Guettarda L.}

G. PARviflora Sw., acc. to Ernst, by which, however, is probably meant G. parvifolia Sw., Fl. Ind. Occ., vol. 3, p. $195 \mathrm{~S}$ (1S06); Griseb., Fl. Brit. IW. Ind., p. 333.- Santa Ana, Ernst.

G. scabra Lam., Tabl. encycl., vol. 2, p. 218, pl. 15t, fig. 3 (1793). -San Juan MIt., alt. 600 m., Johnston, no. 114, July 19. Tree, 6 m. high; wood very brittle. Distribution general in tropical America.

\section{Hillia Jacq.}

H. Parasitica Jacq., Enum. pl. Carib., p. $1 \mathrm{~S}$ (1760), and Hist. Stirp. Am., p. 96, pl. 66. H. longiflora Sw., Prod. reg. Ind. Occ., p. 5S (1788); Griseb., Fl. Brit. W. Ind., p. 325.-San Juan MIt., alt. 550 m., Johnston, no. 41, July 16 . Shrub, 2-1 m. high; flowers waxy, white. Further distribution, Mexico, West Indies, Colombia.

\section{Psrchotria L.}

P. Glabrata Sw., Prod. veg. Ind. Oce., p. 43 (17SS); Griseb., Fl. Brit. IV. Ind., p. 341.-El Valle, Miller \& Johnston, no. 215, Aug. 15; Juan Griego trail, alt. 450 m., Johnston, no. 337, Aug. 12-15. Further distribution, Jamaica, Antigua, Cuba.

P. Horizontalis Sw., Prod. reg. Ind. Occ., p. 44 (17SS); Griseb., Fl. Brit. W. Ind., p. 344.-El Valle, Miller \& Johnston, no. 49, July 27. Further distribution, tropical America.

\section{RaNdia L.}

R. Aculeata L., Sp. pl., vol. 2, p. 1192 (1753); Griseb., Fl. Brit. IV. Ind., p. 31S.- South Hill, El Valle, Johnston, no. 2S0, Aug. 31. Venezuela: Ernst in Seem., Journ. bot., vol. 5, p. 292 (1S67). Distribution general in West Indies.

\section{Sperifacoce L.}

S. Texcion L., Sp. pl., vol. 1, p. 102 (1753) excl. syn. Dill.; Griseb., Fl. Brit. W. Ind., p. 349.-El Valle, Miller \& Johnston, no. 198, July 15; Juan Griego trail, Johnston, no. 99, Aug. 12-15. Widely distributed in tropical America. 
S. verticillata L., Sp. pl., vol. 1, p. 102 (1753).- Santa Ana, Ernst. Distributed in tropical America and Africa.

\section{CUCURBITACEAE.}

\section{Anguria L.}

A. umbrosa HBK., Nov. gen. et sp., vol. 2, p. 121 (1817); Griseb., Fl. Brit. W. Ind., p. 289.- Juan Griego trail, alt. 450 m., Johnston, no. 2S7, Aug. 12-15. Tenezuela: near Bordones and Cumaná, HBK., l. c. Further distribution, Mexico, Trinidad, Venezuela.

ANGURIOPSIS Johnston.

A. margaritensis Jolmston, Proc. Amer. acad. arts and sci., vol. 40, p. 697 (1905).--Along the roadside from El Valle to San Antonio, Johnston, no. 2S6, Aug. S. Climbing over such shrubs as Lycium salsum and Cereus eburneus.

\section{Ceratosanthes Adans.}

C. tuberosa J. F. Gmel., Srst., p. 102 (1791); Griseb., Fl. Brit. W. Ind., p. 259.- El Valle, Miller \& Johnston, nos. 25S, 248; Tacarigua, Johnston, no. 62, Aug. 1t. Further distribution, Grenada, Martinique, Venezuela.

\section{Citrullus Forsk.}

C. vulgaris Schrad. ex Eckl. \& Zeyh., Enum., p. 279 (1835); Duthie \& Fuller, Field and gard. crop, vol. 2, pls. 55, 56.-Cultivated.

\section{Cucumis L.}

C. Melo L. Sp. pl., vol. 2, p. 1011 (1753).- El Valle, Miller \& Johnston; no. S7. Cultivated.

C. Anguria L., Sp. pl., vol. 2, p. 1011 (1753); Griseb., Fl. Brit. IV. Ind., p. 2ss; Mart., Fl. Bras., vol. 6, pt. 4, p. 16.-El Valle, Miller \& Johnston, no. 224, July 14. Distribution general in tropical America.

\section{Cucurbita L.}

C. Pepo L., Sp. pl., vol. 2, p. 1010 (1753); Desc., Ant., vol. 5, pl. 323.- Cultivated, according to Ernst. 


\section{Melothria L.}

M. Pervaga (Macf.) Griseb., Fl. Brit. IT. Ind., p. $2 \$ 9$ (1S60). Landersia pervaga Macf., Fl. Jamaic., vol. 2, p. 142 (1837).- Santa Ana, Ernst. Distribution general in tropical America.

\section{Momordich L.}

M. Charantia L., Sp. pl., vol. 2, p. 1009 (1753); Griseb., Fl. Brit. W. Ind., p. 287.- Santa Ana, Ernst; El Valle, Miller \& Johnston, no. 86 , July 12 . Widely distributed in tropical countries.

\section{CAMPANULACEAE.}

\section{Centropogon Presl.}

C. surinamensis (L.) Presl, Prod. monog. Lobel., p. 48 (1S36); Paxt., Mag., vol. 13, p. 149; Griseb., Fl. Brit. W. Ind., p. $385 . \quad$ Lobelia surinamensis L., Sp. pl., ed. 2, vol. 2, p. 1320 (1763).- San Juan Mt., alt. $750 \mathrm{~m}$. , Johnston, no. SS, July 6. Further distribution, St. Vincent, Trinidad, and tropical South America.

\section{GOODENIACEAE.}

\section{Scaevola L.}

S. Plumieri Vahl, Symb. bot., rol. 2, p. 36 (1791); Griseb., Fl. Brit. W. Ind., p. 3SS. - Pt. Mosquito, Johnston, no. 319, Aug. 10. Venezuela: Tortuga, Emst, Fl. Chelon. in Journ. bot., vol. 14, p. 178. Widely distributed in tropical countries.

\section{COMPOSITAE.}

Acanthospermum Schrank.

A. australe (Loefl.) Ktze., Rev. gen., vol. 1, p. 303 (1S91). A. brasilum Schrank, Pl. rar. hort. Monac., vol. 2, p. 53 (1819). A. xanthioides DC., Prod., vol. 5, p. 521 (1836); Griseb., Fl. Brit. W. Ind., p. 369. Melam podium australe Loefl., It. Hisp., p. 268 (175s).Santa Ana, Ernst. Further distribution, West Indies, Venezuela, Guiana to Buenos Ayres. 


\section{Ageratum L.}

A. conrzoides L., Sp. pl., vol. 2, p. $\$ 39$ (1753); Griseb., Fl. Brit. W. Ind., p. 356.- Santa Ana, Ernst. Common in tropical countries.

\section{BACCHARIS L.}

B. Rhexioldes HBK., Nov. gen. et sp., vol. 4, p. 66 (1820).- El Valle, Miller \& Johnston, no. 230, July 30; San Juan Mt., alt. 600 m., Johnston, no. 98, Aug. 28. Further distribution, Peru, Brazil.

\section{Bidens L.}

B. PILOSA L., Sp. pl., vol. 1, p. 832 (1753). B. leucantha Willd., Sp. pl., vol. 3, p. 1719 (1804); Plum., Ic. Burm., pl. 53; Griseb., Fl. Brit. W. Ind., p. 373.- Santa Ana, Ernst. Distribution general in tropical countries.

\section{Blainvillea Cass.}

B. BAHiensis (DC.) Baker in Mart., Fl. Bras., vol. 6, pt. 3, p. 177, pl. 57 (1882). Oligogyme bahiensis DC., Prod., vol. 5, p. 629 (1836).El Valle, Miller \& Johnston, no. 131, July 20. Further distribution, Brazil.

\section{Clibadium L.}

C. surinanensis L., Mant., vol. 2, p. 294 (1771). C. asperum DC., Prod., vol. 5, p. 506 (1836); Griseb., Fl. Brit. W. Ind., p. 367.- Santa Ana, Ernst. Distribution general in tropical America.

Eclipta L.

E. Alba (L.) Hassk., Pl. Jav. rar., p. 528 (1848); Griseb., Fl. Brit. W. Ind., p. 370. E. erecta L., Mant., vol. 2, p. 286 (1771). Verbesina alba L., Sp. pl., vol. 2, p. 902 (1753).-Santa Ana, Ernst; El Valle, Miller \& Johnston, no. 241, July 30. Widely distributed in temperate and tropical countries.

\section{Elephantopus L.}

E. spicatus B. Juss. ex Aubl., Pl. Guian., vol. 2, p. 808 (1775). Distreptus spicatus Cass., Dict. sci. nat., vol. 13, p. 367 (1819); Griseb., Fl. Brit. W. Ind., p. 355.- Santa Ana, Ernst. Venezuela: Caracas, according to Sch. Bip., Linnaea, vol. 20, p. 519. Widely distributed in tropical America. 


\section{Eleutheranthera Poit.}

E. ovata Poit. ex Steud., Nom., ed. 2, vol. 1, p. 549 (1841). - El Valle, Miller \& Johnston, no. 132, July 18. Distributed in tropical America.

\section{Erigeron L.}

E. spathulatus Vahl in West, Bidr. Ste-Croix, p. 303 (1793); Griseb., Fl. Brit. W. Ind., p. 365.- Rio Asuncion, alt. 450 m., Johnston, no. 324, July 29. Further distribution, Antigua, and Virgin Ids.

\section{Eupatorium L.}

E. ballotaefolium HBK., Nov. gen. et sp., vol. 4, p. 121 (1S20); Mart., Fl. Bras., vol. 6, pt. 2, p. 380.-El Valle, Willer \& Johnston, no. 234, July 15; San Juan Mt., alt. 500 m., Johnston, no. 94, July 19. Venezuela: Colonia Tovar, Fendler, no. 653, 1854-55; La Guaira, Robinson \& Lyon, July 12, 1900. Further distribution, Colombia, Brazil.

E. IREsinoides HBK., Nov. gen. et sp., vol. 4, p. 106, pl. 340 (1820); Griseb., Fl. Brit. W. Ind., p. 360.- El Valle, Miller \& Johnston, no. 129, July 30; San Juan Mt., alt. 500 m., Johnston, no. 95, July 19. Widely distributed in West Indies, Panama, Yenezuela. A thick shrub, 2-3 m. high.

\section{ISOCARPHA R. Br.}

I. oppositifolia R. Br., Trans. Linn. soc., vol. 12, p. 110 (1816); Griseb., Fl. Brit. W. Ind., p. 376.- El Valle, Miller \& Johnston, no. 138, July 10. Further distribution, Bahamas, Jamaica, Trinidad.

\section{LACTUCA L.}

L. INTYBaCEA Jacq., Ic. pl. rar., vol. 1, pl. 162 (1781-1786). Brachyramphus intybaceus DC., Prod. vol. 7, pt. 1, p. 177 (1838); Griseb., Fl. Brit. W. Ind., p. 384.- El Valle, Miller \& Johnston, no. 25, July 27, and Johnston, no. 351, Aug. 12-15. Widely distributed in tropical countries.

\section{Mikania Willd.}

M. AMARA Willd., Sp. pl., vol. 3, p. 1744 (1804).- En route El Valle to Juan Griego, Johnston, no. 104, Aug. 12-15. Further distribution, Martinique, Bolivia. 


\section{Parthenium L.}

P. Hysterophorum L., Sp. pl., vol. 2, p. 988 (1753); Griseb., Fl. Brit. IV. Ind., p. 369.- Santa Ana, Ernst; EI Valle, Miller \& Johnston, no. 233. Tenezuela: near Caracas and on the banks of the Orinoco, HBK., Nov, gen. et sp., vol. 4, p. 259. Widely distributed in temperate and tropical America.

\section{Pluchea Cass.}

P. odorata (L.) Cass., Dict. sci. nat., vol. 42, p. 3 (1826); Griseb., Fl. Brit. IV. Ind., p. 366 . Conyza odorata L., Syst. nat., ed. 10, vol. 2, p. 1213 (1760).-Santa Ana, Ernst; El Valle, Miller \& Johnston, no. 1, July 21, and Johnston, no. 318. Distribution general in tropical America.

\section{Porophyllui Adans.}

P. ruderale (Jacq.) Cass., Dict. sci. nat., vol. 43, p. 56 (1826); Griseb., Fl. Brit. IV. Ind., p. 379. Kleinia muderalis Jacq., Enum. pl. Carib., p. 28 (1760), as rudealis.-Santa Ana, Ernst; El Valle, Miller \& Johnston, no. 130, July 24. Distribution general in tropical America.

\section{Senecio I.}

S. sonchifolia (L.) Moench, Meth. suppl., p. 231 (1S02). Cacalia sonchifolia L., Sp. pl., vol. 2, p. 835 (1753). Emilia sonchifolia DC., Prod., vol. 6, p. 302 (1837).- El Valle, Miller \& Johnston, no. 137, July 19. Widely distributed in tropical countries.

\section{Sonchus L.}

S. oleraceus L., Sp. pl., vol. 2, p. 794 (1753); Griseb., Fl. Brit. W. Ind., p. 384.- Santa Ana, Ernst. Widely distributed in temperate and tropical countries.

\section{Spilanthes Jacq.}

S. ocyuifolia (Lam.) A. H. Moore, Proc. Amer. acad. arts and sci., vol. 33, p. 531 (1907). Bidens ocymifolia Lam., Encycl., vol. 1, p. 416 (1783). Spilanthus exasperata Jacq., Ic. pl. rar., vol. 3, p. 15, pl. 584 (1786-93).- Rio Asuncion, alt. 450 m., Johnston, no. 102, Aug. 12-15. Venezuela: Caracas, Birschel; Colonia Tovar, Fendler, no. 691, 1854-55. Distribution general in tropical America. 


\section{SrnedRElla Gaertn.}

S. NoDiflora Gaertn., Fruct., vol. 2, p. 456, pl. 171 (1791); Griseb., Fl. Brit. IV. Ind., p. 377.- Santa Ana, Ernst. Distribution genera in tropical America.

Trixis P. Br.

T. Radiale (L.) Lag., Amen. nat., vol. 1, p. 36, in obs. (1811), combination implied but not specifically made; Hook. f. \& Jacks., Ind. Kew., vol. 2, p. 1131 (1895). Perdicium radiale L., Sp. pl., ed.'2, vol. 2, p. 1248 (1763). Trixis frutescens P. Br. ex Spreng., Syst., vol. 3, p. 501 (1S26); Griseb., Fl. Brit. W. Ind., p. 383; Ernst, Bot. Excurs. Margarita, p. 6.- Santa Ana, Ernst; El Valle, Miller \& Johnston, no. 24, Aug. 1. Widely distributed in tropical America.

\section{Verbesina L.}

V. Alata L., Sp. pl., vol. 2, p. 901 (1753); Griseb., Fl. Brit. W. Ind., p. 374; Sims., Bot. mag., pl. 1716.- Santa Ana, Ernst. Distribution general in tropical America.

\section{Vernonia Schreb.}

V. Arborescens (L.) Sw., Fl. Ind. Occ., vol. 3, p. 1320 (1S06); Griseb., Fl. Brit. W. Ind., p. 353; Plum., Ic. Burm., pl. 130, fig. 2. Conyza arboreseens L., Syst. nat., ed. 10, vol. 2, p. 1213 (1759).- San Juan Mt., alt. 500 m., Johnston, no. 96, July 19. Flowers blue; stem 2-3 m. high. Distribution general in tropical America.

V. Milleri Johnston, Proc. Amer. acad. arts and sci., vol. 40, p. 698 (1905).- Summit of South Hill, alt. 300 m., Miller \& Johnston, no. 254, July 31, and Johnston, no. 329, Aug. 31. Endemic.

V. scorpioides (Lam.) Pers., Syn., vol. 2, p. $40 t$ (1S07); Griseb., Fl. Brit. W. Ind., p. 353. Conyza scorpioides Lam., Encycl., vol. 2, p. SS (1786). - El Valle, Miller \& Johnston, nos. 133, 134, July; Juan Griego trail, Johnston, nos. 322, 323, July. Venezuela: Caracas, according to Sch. Bip., Linnaea, vol. 20, p. 510. Further distribution, Trinidad and tropical South America.

\section{IVedelia Jacq.}

W. Buphthalviomes Griseb., Goett. Abh., vol. 7, p. 235 (18.57).-El Valle, Miller \& Johnston, nos. 145, 146, and 147, July and Aug. 
Shrubby, spreading, 0.3 to $1 \mathrm{~m}$. high. Further distribution, West Indies.

IV. Caracasana DC. Prod., vol. 5, p. 541 (1836); Griseb., Fl. Brit. W. Ind., p. 371. - San Juan Mt., alt. 600 m., Johnston, no. 97, July 11. Venezuela: near Caracas, Vargas, no. 97, 1829; Birschel; A. II. Moore, Mar. 16, 1899; Colonia Tovar, Fendler, nos. 682, 683 , in 1851-55, and 1961, Jan. 25, 1857; La Guaira, Robinson \& Lyon, July 6. Further distribution, Trinidad, Panama.

WulfFia Neck.

IV. Baccata (L. f.) Ktze., Rev. gen., vol. 1, p. 373 (1891). Coreopsis baccata L. f., Suppl., p. 380 (1781). Helianthus ? sarmentosus Rich., Act. soc. nat. hist. Par., vol. 1, p. 112 (1792). Wulffia stenoglossa DC., Prod., vol. 5, p. 563 (1\$36); Griseb., Fl. Brit. W. Ind., p. 372.-San Juan Mt., alt. 500 m., Johnston, no. 101, July 19. Bush, 1-1.5 m. high. Further distribution, West Indies, Guiana to Peru.

\section{Economic Plants of Margarita.}

The most important of the plants, which are cultivated on Margarita, is the coconut palm. Although there are only the two large groves, one completely filling the lower part of El Valle and the other extending through Asuncion valley, yet they furnish a large part of the coconuts used on the island. The huts of the poor people are constructed of the leaves, and the fruit constitutes an important part of their food. The cultivation of these groves consists merely in keeping the undergrowth down and in maintaining irrigation ditches.

'The next most important vegetable product of the island is cassava. made from Manihot utilissima. Fields of this are usually upon a hillside and it is abundantly grown in such places in El Valle. There is, however, in Asuncion, one large field of cassava on the plain. The cultivation of this plant on the hillside, which is preferable on account of loose soil and good drainage, is rendered difficult by the steepness of the slopes. Planting is done by setting out cuttings from the stem each about $15 \mathrm{~cm}$. (6 in.) long, that are placed in rows about $1.5 \mathrm{~m}$. apart. The earth is heaped in small ridges between the rows and the plants so as to form ridges about each plant, thus serving to hold whatever water may come down. In some parts, a single row of stones extends between each two rows of plants, in that way forming 
a sort of a stone wall about each plant. This contrivance is to prevent the heary rains from washing all the soil down the hillside. When the crops are mature, the roots are gathered and treated in the customary way, bruised, mashed, and the poisonous juice pressed out. The dried remainder is grated into meal and made into cassava bread which is sold in large, thin, circular disks.

Indian corn is grown very little upon the island, and then it is used mostly for fodder. A few acres were given up to its cultivation at an altitude of $500 \mathrm{~m}$. in 1901 . In 1903, however, the same space was entirely occupied by a rank growth of Cordia cylindristachya and other weeds.

The bread-fruit tree, Artocarpus incisa, is to be found in Asuncion valley. Bananas and plantains are to be had in moderate amount and only fair in quality.

Dates grow in sufficient abundance for the needs of the natives. There are probably a half dozen mature trees in El Valle. The foot of the mountain with its moist soil and extremely hot atmosphere is a good place for growing the trees.

The cashew-nut (Anacardium occidentale) and the hog-plum (Spondias lutea) are to be found sparingly near Asuncion.

Maranta arundinacea, the arrowroot plant, grows in the woods above Asuncion but it is not eultivated.

The mamon (Melicocca bijuga) and the toco (Crataeva Tapia) are often eaten and sometimes found for sale in the market-place.

The mango is the most abundant fruit in Margarita and though of inferior quality is largely used by all the people.

There are orange trees in El Valle, Asuncion, and Tacarigua, those from the last place being superior. The few bitter orange and the lime trees are of little importance.

Good sapodillas or nisperos grow both in El Valle and at Asuncion. A poor quality of pineapple is to be found in the market in season. There are several pineapple fields on the hillsides of El Valle.

Sugar cane grows well in very few places on the island. There are several small cane-presses, turned either by hand or by burro. The product is for home consumption, and there is not enough of it to supply the people. Most of the sugar that is used is brought over from Cumana in the form of large brown cakes called papelon. The cane-juice may be found in the market in a slightly fermented condition when it is known as guarapo. 
'The following fruits and vegetables grow in El Valle and at Asuncion though sparingly, not being cultivated to any extent: Annona reticulata, the custard-apple; Malpighia punicifolia a small fruit not used much; Cajanns indicns, the pigeon pea; Dolichos sp., a pea; Phaseolus vulgaris, a bean; Psidium grajava, from which guava jelly is made; Passiflora laurifolia, the belle-apple; Dioscorea alata, a yam; Cucumis Melo and C. Anguria, both small melons; Physalis peruriana, the "tomato"; Tamarindus indica, the tamarind, quite abundant in El Valle.

The foregoing plants may be considered as the only ones of much economic importance to the inhabitants; that is, they are the only ones that are cultivated or made much use of. As a matter of fact the number of plants that are found growing on Margarita and that are important commercially on the mainland and elsewhere is rather large. The list contains a few specimens of timber trees, of medicinal plants, of plants producing resins and gums, and other products such as dyes and tannins. The fact that these do grow upon Margarita suggests that they might be cultivated in greater abundance. That they are not found in greater quantities, while of course due largely to the physical conditions of the island, is also owing to the ignorance and lack of eare on the part of the inhabitants. This is strikingly illustrated in two or three small private haciendas where such things as grapes, egro-plants, tomatoes, and peppers are successfully cultivated. The fact that so many of the plants furnish useful products suggests the possibility that Margarita may be made more productive than it is at present; for that reason it is desirable to enumerate them here together with their uses.

Material for the following has largely been drawn from La Exposicion nacional de Venezuela en 1883 by A. Ernst; the World's Columbian Exposition in Chicago - Venezuela; and Medicinal plants of Caracas by A. Ernst in Seemann's Journal of botany for 1865 .

\section{IIOODS.}

Anacardium occidentale. Wood strong and durable. Little used, as its fruit is of more importance. Trees scarce.

Achras Zapota. Nispero. Wood very hard, strong, and heavy, of a reddish color and taking a beautiful polish. Chiefly valued for its fruit. Trees abundant. 
Bourreria exsucca. Wood good but little used. This is a small tree averaging between 3 and $5 \mathrm{~m}$. in height. Fairly common.

Bursera simaruba. Soft and resinous wood. Common on hilltop.

Caesalpinia cortaria. Dividive. On the mainland a large tree reaching sometimes a height of $30 \mathrm{~m}$. with a clear trunk $10 \mathrm{~m}$. high. On Margarita and on Coche it occurs as bushes about $2 \mathrm{~m}$. high. The wood is very heavy, strong, and fine-grained. The sapwood is whitish yellow, the heartwood black and almost as hard as iron. Rather scarce.

Capparis pachaca. Ajicito. A small tree with a light-colored wood of not much hardness, used for cabinet work. Very few trees.

Cassia fistula. Cañafistula. Wood of a flesh-color, heavy, hard, and strong but not much used. Few trees.

Clusea rosea. Copey. Wood of a reddish color, rather heavy, used considerably on Margarita for building-posts, but not much elsewhere. Common.

Cordia AlBa. Canjaro. Attains a height of 12 to $15 \mathrm{~m}$. with a trunk 4 to $5 \mathrm{~m}$. high. A tolerably good wood for building purposes. Only a few trees.

Crataeva grvandra. Toco. Very light and soft, generally of a whitish color though sometimes showing reddish veins. Sawn into boards for boxes. Common in El Valle.

Crescentia Cujete. Taparo. Wood yellowish white, closegrained, and rather hard and strong. The tree is of medium size. Common.

Guajacli arboreum. Vera. A large tree vielding a wood similar to the following.

Guajacum officinale. Guayacan. Tood exceedingly hard and tough, the fibers erossing each other, so that it does not split. The sap wood is of a yellowish color; the heart wood is brown with dark greenish veins and markings. Used for turnery work. Both of these trees are abundant.

Guazuma ulmifolia. Guacimo. Of a grayish white color with a somewhat reddish hue and occasionally some dark veins; it is fibrous, not very fine-grained, and comparatively light.

Hipponane Mancinelda. Manzanilla de playa. Good wood, durable and heavy, fine grain, annual rings distinet, and pores numerous. Few trees.

Hura crepitans. Jobillo. Wood light and soft, of fibrous texture, durable under water, and color white. Few trees. 
Hymenaed Courbaril. Algarrobo. A very hard and heavy wood, of dark yellowish color with some greenish veins; it has straight fibers and is free of knots, so that it can be easily worked. It is used especially for crushing-wheels and similar things in coffee estates. Height usually 20 to $25 \mathrm{~m}$., with a clear trunk 7 to $8 \mathrm{~m}$. On Margarita, however, the only specimens observed were very low trees.

LAGUNCULARIA RACEnOSA. Mangle amarillo. Wood hard and heavy, fibrous and compact in texture. Color gray with many black points. Common about the lagoons.

Lonchocarpus sP. Mahomo. Very hard and elastic. The species on Margarita are from 15 to $20 \mathrm{~m}$. high. Few trees.

Malphigia 'PUNicifolia. Wood very compact, light, not very durable, of a clear brown color. Few trees.

Melicocca BiJugA. Mamon. Wood hard and heavy, compact and close-grained, color yellowish with very narrow and somewhat darker veins. Its fruit is usually valued more highly than the wood. Common.

Morisonia SP. Wood white, soft and light. Scarce.

NECTANDRA CORIACEA. Tery strong and resistent, not heavy, with good grain, and a more or less agreeable odor. Is easy to work, being used in carpentry and cabinet work. Scarce.

Oreopaxax capitatum. Candelero. Wood whitish, brittle and light. Searee.

Pisonia Ineruis. Wood of little weight, light in color, good for use under water. Common.

Rhizophora Mangle. Mangle colorado. Wood red, strong, and heavy. Much used for rafters. Very abundant about lagoons.

Tabernamontana psychotrifolia. Berraco. The wood is fibrous, not very hard, and of an olive color, sometimes with darker veins. It is easily worked and takes a good polish. Common.

Other trees found in small numbers on Margarita and not much used are Inga ingoldes, Guettarda scabra, Morisonia Johnstonil, ACACIA MACRACANTHA, and LiNOCIERA LATIFOLIA.

\section{FIBER PLANTS.}

All of the following grow rather sparsely in Margarita:-

Agave americana. Cocmy.

Bолвах сепв. Ceiba. The fibers are short and are used solely for stuffing pillows, etc. 
Bromelia Pinguin. Maya.

Gossipium barbadense. Algodon. Many hammocks are handmade from cotton on Margarita. See Ernst: La Exposicion nacional de Venezuela en 1S83, pages 560, 561 for quotation describing method of making hammocks according to A. A. Level, La Margarita, pages Ixviii and lxix.

Musa paradisiaca. Platano.

Tournefortia hirsutissiala. Niguo.

Tragia volubilis. Pringamosa Morada.

Palia Carana so called by the natives and mentioned by Ernst. Its specific identity is doubtful. The leaves are used to make brooms, ropes, etc.

\section{GUMS AND RESINS.}

Achras Zapota. Gum Chicle. Milk juice obtained by incision in bark. Tapping may occur once in three years without danger to life of tree. The raw milk is boiled and then allowed to harden into the gum. Used for chewing gum.

Bursera gummitera. Resina indio desmudo.

Cercidium viride. Res na de cuica ó yabo. This resin exudes from the bark of the stem and branches, covering them in a continuous layer. It is used by soap manufacturers.

Clusea rosea. Resina de Copey.

Hrienaea Courbaril. Resina de algarrobo. The resin exudes from the stem and roots of the tree and is often found in a semi-fluid state in the soil. It may be used for making varnish, like copal. Is used for incense.

Spondias lutea. Goma de jobo.

\section{DYES AND TANNIN.}

Bixa orellana. Onoto. The seeds are covered with a deep red pulp which hardens when dry, and being separated from the seeds forms the arnatto of commerce, used by dyers and varnish-makers also for coloring cheese and butter. The South American Indians paint their bodies with it.

Caesalpinia coriaria. Dividive. The pods contain from 30 to $40 \%$ tannin and form an important article of exportation from several ports of Venezuela. 
Cecropia peltata. The bark may be used for tanning.

INDIGOFERA SUFFRUTICOSA. Formerly much used in making indigo.

Persea Gratissima. The bark of this might be used for tanning leather.

RH:zophora MANGle. Contains from 22 to $33 \%$ of tannin according to the age of the tree, and likewise a pigment which colors the leather.

\section{MEDICINAL PLANTS.}

Abrus pRECATORIUs. Substitute for licorice in India. Is a poison and a medicine.

Acacia inacracantha. Corteza de Cuji. Astringent.

Agave americana. Raiz de Cocuy. Purgative.

Anacardium occidentale. Root is purgative, the green fruit astringent. In the middle layer of shell of fruit is an oily liquid capable of poisoning the skin, and turning black on exposure. This is used as an indelible ink.

ANNoNa muricata. Root yields a fish poison.

AnNona squamosa. Bark has drastic properties.

Argemone mexicana. Cardo santo. Used as a mild cathartic and in cases of intermittent ferer, and dropsy.

Aristolochia ringens. Congrina. For rheumatism.

Asclepias curassavica. The root is a diuretic and carminative, in large doses a cathartic and an emetic.

Bastardia viscosa. Chivatera 6 fistulera. Aromatic. The powder is said to cure fistula.

Bixa orellana. The red arillus is used in soups and sauces.

Brunfelsia Hopeana. The dried root and stem used for chronic muscular rheumatism.

Bursera gumifera. Indio desmudo. Used for rhelumatism.

Calipyloneuruir phyllitidis. Lengua de sierpe. Antisyphilitic.

Capparis Jamaicensis and Capparis cynophallophora. Root bark possesses blistering properties and may be taken internally as a diuretic.

Capsicun baccatun. The berries used as caustics, and the leaves bruised and mixed with tallow, are applied to tumors to promote suppuration.

Capraria biflora. The root is a tonic. 
Carica Papaya. The juice from the fruit aids in digestion.

CAssia BIFloRA. Leaves soaked in water make a refreshing draught, principally taken in fever caused by insolation, but also in intermittents.

Cassia fistula. Cañafistula. Mild purgative.

Cassia occidentalis. Brusca. Febrifuge and astringent. The seeds roasted give a drink similar to coffee.

Ceratosanthes tuberosa. Raiz de pepino. Emetic.

Cissampelos Pareira. Diuretic.

Cordia gerascanthus. Cantaro. Sudorific and pectoral.

Costus spicatus. Raiz de Caña de la India. Antisiphilitic.

Craniolaria anvua. Escorzonera. The seeds are used against the irregularities of the blood, the root is a purgative and cooling.

Dactrlocteniun aegrptum. Raiz de Guarataro. Diuretic.

Dieffenbachia seguine. Decoction made from stem, thickened with gum arabic is used in cases of gonorrhea.

Guazuxia ulmifolia. Guasimo. Mucilaginous, refreshing and healing.

Hippoilane Mancinella. Fish poison from juice. Caustic, emetic, cathartic.

Hrmenaea Courbaril. The dried pulp of the fruit is a pectoral. Jatropha Curcas. Pinon. Emetic.

JAtropha gossypifolia. Tivatua. Emetic.

Lantana Camara. Cariaquito encarnado. Diuretic and refreshing. The root is used against gonorrhea.

Mangifera INDiCA. Seed is anthelmintic; juice of trunk antisvphilitic; bark and leares astringent.

Melia Azedarach. Decoction of bark is an anthelmintic for the removal of the round worm.

Melochia tomentosa. Bretonica. Used in the infirmities of the eyes.

Parthenium Hysterophorum. Large doses have an antipyretic effect.

Pedilanthus tithrialoides. The milky sap is an emetic.

Persea gratissina. Astringent kernel, eut in pieces, roasted, and reduced to powder, good for diarrhea and dysentery.

Petiverla alliacea. Raiz de Mapurite. Antispasmodic and vermifuge.

Phyllanthus Niruri. Laxative and alterative, especially against jaundice. 
Plumbago scandens. Guapote. Rubefacient.

Portulaca oleracea. Decoction is anthelmintic and refreshing. Portulaca pilosa. Verdolaga salvaje. A bitter and tonic.

Scoparia Dulcis. Raiz de escobilla. A decoction used to restrain diarrhea and vomiting.

Sida RHonifolia. Escoba blanca 6 babosa. The root is a diuretic.

Spondias lutea. Corteza de Jobo. A decoction is used to cauterize stubborn ulcers.

Stachytarpheta Jamaicensis. Verbena. A bitter, tonic, and febrifuge.

Trixis radiale. Juan de la Calle. Antirheumatic.

Turnera diffusa. Contains damiana, a stimulant tonic; in large quantities a laxative.

Turnera ulmifolia. Carminative and tonic.

\section{Distribution of the Plants.}

It may easily be seen from the description of the physical features of the island that the variety of the vegetative conditions is very great and also that the conditions for vegetative growth are much more farorable in the rainy than in the dry season. The distribution of the plants is naturally determined by their adaptation to particular conditions.

Bordering the sandy beaches are bushes of Tournefortia gnaphalodes and Suriana maritima, both of these being narrow-leaved, and the former being woolly-pubescent. Croton flacens is a similar plant. Next inland occur the low spiny bushes of Castela Nicholsoni. The small and long-rooted Euphorbia buxifolia grows in the sand dunes and near by are the small trees of Bumelia cuneata and Guaiacum officinale. On the exposed rocky shores by Juan Griego, Euphorbia thymifolia grows in abundance in almost no soil.

The region nearest in character to the seashore is the lagoon. This is often bordered by Rhizophora Mangle, Laguncularia racemosa, and Avicennia nitida, all shrubby or aborescent plants. On clear sandy stretches by the lagoons and sometimes partly submerged in the water are the low succulent plants, Batis, Salicornia, and Trianthema. Mingled with these in the drier places are Alternanthera canescens and Iresine portulacoides, both having a low sprawling habit. On the muddy shores of the brackish pond near Juan Griego grow the 
tiny Cypselea humifusa, Euphorbia thymifolia, Ilysanthes riparia; and small specimens of Mollugo verticillata.

Inland from these wastes are the plains covered with the melon cactus, the flat-stemmed Opuntia, the symmetrical tree-like Pereskia, and the candelabra-shaped, tall Cereus eburneus. All these are thorny and more or less fleshy plants. In addition are the arborescent acacias, and Capparis cynophallophora; the shrubby Croton flacens, Croton Milleri, Jatropha gossypifolia, and Jatropha urens, and the small shrub Stylosanthes.

The desolation of the plains is emphasized on the hills by the addition of great stretches of Agave and Aloe which make the regions almost inaccessible. The high parts of the hills have dense growths of bushes, as Cordia cylindristachya, C. globosa, Securidaca, Capparis verrucosa, and Heteropteris lanrifolia. Then scattered over the hills are the small trees of Capparis cynophallophora, C. lincaris, Bursera, and Steriphoma. Along the hilltops are a few larger trees of Morisonia and Clusia. These trees have leathery and rery large leaves in all cases except Bursera and Capparis linearis. The undergrowth among the trees on the hills consists of the green-stemmed Pedilanthus, the bushy Brunfelsia, and the bromeliaceous plants Aechmea and Thecophyllum. Here are a few epiphytes as Oncidium, the cactus Rhipsalis, a few Polypodiums, some of the Bromeliaceae, and Anthurium scandens.

The vegetation of the valleys, with the exception of Asuncion and El Valle, is similar in general to that of the hills and the plains. These two valleys have groves of coconut, mango, sapodilla, and orange trees, and in consequence afford a good place for the growth of annual plants. The river beds and arroyas are the most interesting places in the valleys. The only large river bed on the island is that extending from the mountain through $\mathrm{El}$ Valle to the sea at Porlamar. It is bordered with such trees as Crataeva Tapia, Guajacum arboreum, Lonchocarpus, Pithecolobium, and Bombax, all with tall gray trunks. There are many bushes, some half-climbers, Chiococea, Cestrum, Malpighia purpurea, Solanum, Acacia, and Marsdenia. The arroyas or gullies high up in the valleys have the small tree Tecoma, the shrub Cordia globosa, and the vine Bignonia.

The mountain furnishes varied situations and conditions for the growth of plants. In general it is forested from $300 \mathrm{~m}$. nearly to the summit; in the valleys the woods grow at a lower altitude also. The 
trees which make up the forest are Bombax, Clusia rosea, Cecropia, Inga, Gilibertia, Linociera, and the palms Acrocomia, Oredoxa, and Bactris, all these being trees of a more or less straight trunk. The crooked trees of $500 \mathrm{~m}$. altitude or more are Guettarda, Hellia, Pisonia, Nectandra, Phoebe, Psychotria, and Clusia lutea. At the summit of the mountain are the dwarfed Clusia lutea, the wide-spreading Blakea, Vaccinium, and Myrcia.

Among the trees of the lower altitudes there is little undergrowth, consisting of the ferns or orchids. At an altitude of $400 \mathrm{~m}$. on the San Juan trail there is a small marshy area covered with Heliconia Bihai and at one side is Acalypha. At an altitude of $500 \mathrm{~m}$. in the Asuncion valley, that is, the northeast side of the island, there is considerable undergrowth. In the woods, Dioscorea, Smilax, Piper; by the "rios," Athyrocarpus, Costus, Calathea, Renealmia; and on the rocks and trees, Philodendron, Anthurium, and Dieffenbachia, together with many terrestrial and epiphytic ferns and orchids, may be found. Various members of the Gramineae are scattered throughout the woods. In open thickly grown places is Scleria bracteata; by rivulets are Cyperus, Scirpus, Elcocharis, and a few other Cyperaceae. Drymonia serrulata is one of the vines among the trees by the "rio." Gonzalugania and Chioeocca micrantha at lower altitudes have slender ascending or climbing habits. Above $500 \mathrm{~m}$. the melastomaceous Cliclemia and Miconia are to be found. The deep ravines are characterized by the abundance of ferns, especially one ravine by the treefern, Cyathea.

The wooded mountain top between 600 and $700 \mathrm{~m}$. has practically no undergrowth. The low trees already mentioned are crooked and crowded together. They are corered with moss which is saturated with moisture. On the trunks may be found Polypodium jubaeforme and Yiphopteris serrulata, but practically nothing else, unless it is Lycopodium and mosses. Above $700 \mathrm{~m}$. or on the exposed part of the mountain top is a great variety of plants. The bromeliaceous genus Glomeropitcairnia is found growing thickly over parts of the top. The delicate Utricularia is abundant in the rich and moist humus. The tiny shrub Sauvagesia, the vine Echites, and the sprawling Epidendrum secundum, the erect Epidendrum nocturnum, the beautiful Centropogon, several passion-flowers, the gentian Coutoubea, the silver fern and several sedges cover the top of the mountain. Such in general is the distribution of the plants according to regions. 
In connection with this discussion there is an interesting field for study in the distribution of the individual species of plants whether in groups or singly, whether in one place or scattered in many, and whether on one slope and not on another.

The species of plants found on the seashore and by the lagoons are without exception growing in groups with but little intermixture. Almost any of the plants illustrate this, Rhizophora, Avicennia, Batis, Salicomia, and Trianthema. The species growing on the plains are found in abundance though well interspersed with other species. So far as was discovered Opuntia leptocaulis was growing in abundance but over only a small area to the west of Porlamar. Similarly, Croton Milleri was localized though well mixed with other plants. MIany of the plants were to be found in different locations, which, however, had similar moisture conditions. This was even more accentuated in the valleys and hillside than on the plains. While many plants could be found in a fairly large quantity, the majority of the hillside plants occurred in small numbers. Some plants which were found only on the south slope of a hill might be found on the south slope of another hill but not on the north side. Further detailed exploration might tend to generalize this statement but the conditions as given were rather striking to me. For example, Steriphoma elliptica occurs on the south slope of South Hill and on the south slope of North Hill but not on the other parts of the island so far as explored. Bauhinia cumanensis occurs similarly. Cases of isolation are Securidaca only on the north side of North Hill, Hymenea Courbaril on the south side of South Hill and Pedilanthus on the summit of South Hill.

The instances in which only two or three plants of a species were found are few and it must be admitted that a thorough examination of the hills and ralleys in a better season would probably show more of the plants. Notwithstanding this the paucity of specimens was only too apparent to me when collecting, and it seems to be a fact that in the majority of cases (striking exceptions are Tribulus, Stachytarpheta, Jatropha, and Croton) the number of plants of the individual species is very much smaller than is the case with our common plants of the United States. Only one plant was found of Chiococca micrantha, a half dozen near together of Securidaca, a single one of Hymenea, a single tree of Acacia macracantha in San Antonio valley and three or four along the river trail of $\mathrm{El}$ Valle, three plants of Oncidium luridum, three of Huntieya, and three of Elleanthus attenuatus. The list 
might easily be extended and with a more thorough study might furnish an interesting question as to the rapidity of multiplication of these plants and as to the means by which they hold their own among the more rapidly multiplying plants. As to the question why certain plants are found on one slope and not on another, the palms as an example may explain. At an altitude of 500 or more meters, palms of various kinds are scattered about among the other forest vegetation. This occurs, however, only on slopes to the northeast, that is, exposed to the northeast trades. The opposite sides at this high altitude present an ordinary forest front undotted by a single palm. Moisture, then, either by its immediate presence or in its relation to the winds very probably is a factor in the distribution of all the plants.

So far as methods of distribution are concerned it may be said that there are very few special adaptations to dispersal. Cenchrus echinatus seems to be the only one adapted for dispersal by means of its prickly fruit, which adheres to animals. The various members of the Bignoniaceae and of the Asclepiadaceae are suited for wind dispersion as are also Gossypium and Bombax. Of course there is no limit to the carrying of seeds by birds from one valley to another so that the absence or presence of moisture is probably the most potent factor restricting the mountain plants to the mountain and the lowland plants to the lowlands.

The further question as to the distribution of the plants according to season is quite as interesting as the distribution of the plants in the various topographical regions. There is a striking difference in the appearance of the plains, the hills, and the valleys as seen in the rainy season and in the dry. In the rainy period the fields are carpeted with green and the bushes and trees are heavy with foliage and bright with blossoms. In the dry season the fields are almost deroid of stick or leaf and many bushes and trees are to every appearance dead. When the rains come on in July or August, Tribulus terrestris and Kalstroemia maxima cover the roadsides and plains; Stachytarpheta coccinea and $S$. jamaiccnsis, Spermacoce tenuior, Argemone mexicana, Asclepias curassavica, and many others form a rank growth of weeds in the coconut groves and cane fields; various shrubs of the hillside, Capparis vermeosa, Cassia emarginata, Banhinia cumanensis, and others are out in leaf and in flower; and the climbing shrubs and vines form a luxuriant growth along the "rio" beds. In the dry season only a few of these plants can be found in flower. It is noteworthy that in several 
cases the flowers appear before the leares on shrubs; for example, Cercidium viride, Gliricidia lutea, several Bignonias, Erythrina, Cassia emarginata, and Pedilanthus tithymaloides.

In pleasing contrast to the plains, the mountain summit is constantly clothed in green regetation and many blossoms are always to be found. The presence the year round of the moisture-laden clouds accounts for this difference between the mountain top and the plains. The latter have only a few months (August to February) of green regetation, with the remainder of the season characterized by gray lifeless bushes and trees and by an utter lack of low herbs.

The foregoing represents in brief the distribution of the plants on Margarita. 'The discussion has been more suggestive than exhaustive. A thorough exploration of the island according to scientific methods in such work would yield $\mathrm{va}^{\prime}$ lable information not particularly in regard to Margarita but from the point of riew of the life history of the plants themselves. As evinced in the above paragraphs the following topics have seemed to the writer of paramount importance in considering the plant distribution: occurrence of the plants in regions of different regetative conditions; occurrence in groups or scatteringly; and the effect of the seasons both on the occurrence of the plants in different regions and on the abundance of the plants.

\section{Composition of the Flora.}

In order to compare the vegetation of Margarita and Coche with that of the adjacent regions in as thorough a way as is desirable, it is necessary to have a full understanding of the composition of the regetation.

The purpose of studying the flora of these islands from an economic as well as a purely scientific point of view, has necessitated cataloguing the cultivated plants. 'These may or may not be native of the region but in either case they are so widely cultivated in all of tropical America and some of them commonly in the East Indies that for the purposes of comparing floras they must be entirely disregarded. Of those that come under this head the following have been included in the catalogue of plants. Although they are not all cultivated in Margarita yet they are in many other places (see Alph. De Candolle, Géographie bot., vol. 2, p. 9S1-9\$3):

Achras Zapota

Agave americana

Anacardium occidentale
Ananas sativa

Annona reticulata

Annona squamosa 
Antigonon leptopus

Artocarpus incisa

Buginvillea spectabilis

Capsicum annuum

Carica papaya

Citrullus vulgaris

Citrus Aurantium

Cocos nucifera

Crescentia Cujete

Cucumis Anguria

Cucumis Melo

Cucurbita Pepo

Dioscorea alata

Ipomoea Batatas

Ipomoea tuberosa

Mangifera indica

Manihot utilissima
Maranta arundinacea

Momordica Charantia

Musa paradisiaca

Nerium oleander

Nicotiana Tabacum

Phaseolus vulgaris

Phoenix dactylifera

Physalis peruviana

Plumbago capensis

Psidium guajava

Quisqualis indica

Ricinus communis

Saccharum officinarum

Spondias lutea

Tamarindus indica

Thevetia nereifolia

Zea Mays

The above list of forty names includes plants cultivated for ornament as well as those of more practical value. Out of the 634 plants of Margarita and Coche this leaves 590 as constituting the wild plants of the islands. But of this number many are found to be plants of widespread distribution. Undoubtedly some are native and yet many have been introduced. Margarita is one of the oldest known and longest settled (1525) parts of America, hence the opportunities for introduction have been great. 'The plants found in this catalogue and more or less common in all tropical countries are as follows:

Achyranthes aspera

Ageratum conyzoides

Amaranthus paniculatus

Amaranthus spinosus

Amaranthus trist is

Biclens pilosa

Caesalpinia pulcherrima

Cajanus indicus

Calotropis procera

Canavallia obtusifolia

Capraria biflora

Capsicum baccatum

Capsicum frutescens

Cassia fistula

Cassia occidentalis

Cassia Tora

Cenchrus echinatus

Chenopodium ambrosioides
Cissampelos pareira

Clitoria Ternatea

Conocarpus erectus

Crotolaria incana

Cyperus distans

Cyperus ligularis (Mariscus)

Dactyloctenium aegyptium

Datura Metel

Datura Tatula

Desmanthus virgatus

Desmodium incanum

Eclipta alba

Eleocharis capitata

Eragrostis ciliaris

Fuirena umbellata

Heliotropium curassavicum

Heliotropium indicum

Hyptis capitata 
Hyptis pectinata

Indigofera suffruticosa

Ipomoea coccinea

Ipomoea Quamoclit

Ipomoea sinuata

Jatropha Curcas

Jussiaea suffruticosa

Laguncularia racemosa

Leonotis nepetaefolia

Leptochloa mucronata

Panicum prostratum

Parkinsonia aculeata

Paspalum conjugatum

Passiflora foetida

Pisonia aculeata
Psidium guajava

Rhizophora Mangle

Sauvagesia erecta

Scaevola Plumieri

Scoparia dulcis

Senecio sonchifolia

Sesuvium portulacastrum

Sonchus oleraceus

Solanum verbascifolium

Sporobolus virginicus

Stachytarpheta jamaicensis

Suriana maritima

Telanthera ficoides

Tribulus cistoides

Ximenia americana

These widely distributed plants are, of course, common enough in most of tropical America in their respective habitats. With the exception of the sedges, grasses, and ferns, most of the above plants are found about the valleys and along the routes of travel. The exceptions are made because the groups mentioned require the moisture that is found in Margarita only above the valleys in the mountains.

Excluding both the cultivated plants and those of general tropical distribution, there are left but 524 whose habitat is restricted to the American tropics. Margarita is a continental island, belonging to Venezuela. The question naturally arises whether the most of its flora pertains to that part of the American tropics or to the West Indies. A study of the remainder of the plants makes it rery evident that the flora still retains its general character in that the majority of the species are common both to the West Indies and to South America. The exceptions to this are worth noting and are as follows: -

\section{Common to West Indies.}

Tillandsia Lescaillei

Epidendrum globosum

Pelexia adnata

Peperomia glabella

Coccoloba excoriata

Loranthus emarginatus

Cypselea humifusa

Annona reticulata

Oxalis Plumieri

Castela Nicholsoni

Heteropteris laurifolia
Common to South America.

Anthurium scandens

Philodendron eximium

Aechmea Fendleri

Gravisia aquilega

Vriesia scalaris

Stromanthe tonckat

Piper pseudo-mollicomum

Alternanthera canescens

Alternanthera muscoides

Loranthus orinocensis

Steriphoma elliptica 
Common to $\Pi$ est Indies.

Adelia Ricinella

Argithamnia candicans

Croton helicoideus

Croton populifolius

Abutilon umbellatum

Wissadula periflocifolia

Clusia flava

Cereus caripensis

Cereus Swartzii

Opuntia leptocaulis

Pereskia opuntiaeflora

Daphnopsis americana

Rotala dentifera

Myrcia coriacea

Bumelia cuneata

Echites secundiflora

Echites umbellata

Tournefortia gnaphalodes

Tournefortia incana

Tournefortia scandens

Rauvolfia Lamarkii

Drymonia serrulata

Psychotria glabrata

Randia aculeata

Erigeron spathulatus

Wedelia buphthalmoides
Common to South America.

Bauhinia cumanensis

Centrosema brasilianum

Cercidium spinosum

Myrospermum frutescens

Esenbeckia pilocarpoides

Sebastiana corniculata

Talisia olivaeformis

Caracasia tremadena

Hybanthus oppositifolius

Rinorea marginata

Cereus eburneus

Cereus Jamacaru

Combretum secundum

Clidemia neglecta

Vaccinium latifolium

Tabernaemontana psychotrifolia

Evolvulus filipes

Cordia discolor

Clerodendron molle

Brunfelsia Hopeana

Lycium salsum

Beyrichia scutellarioides

Macfadyena corymbosa

Jacobinia pauciflora

Baccharis rhexioides

Eupatorium ballotaefolium

Vernonia scorpioides

Wedelia caracasana

Maxillaria virguneula

In this list of the plants of limited distribution there are thirtyseren to be found in the West Indies or Mexico and forty in South America. 'l'o this last number there should be added the forty-two endemic species. The flora of Margarita may then be said to eonsist of the following elements in the given proportions:-

\begin{tabular}{lr} 
Cultivated plants & 40 \\
Cosmopolitan plants & 66 \\
Common to tropical America & 419 \\
Limited to West Indies & 37 \\
Limited to South America & S2 \\
\multicolumn{1}{c}{ Total } & -644
\end{tabular}

The great variety of plants in Margarita is for the most part in widely separated genera and families. It is seldom that there are 
more than two or three species in any one genus. The well marked exceptions to this are the large tropical genera Panicum, Epidendrum, Piper, Euphorbia, Croton, Cassia, and Solanum. The 644 species are distributed among $39 S$ genera and in $9 S$ families, the larger proportion being among the choripetalous groups.

The families having the largest representation in the flora are as follows:-

$\begin{array}{lcc} & \text { Genera } & \text { Species } \\ \text { Leguminosae } & 41 & 65 \\ \text { Compositae } & 25 & 30 \\ \text { Euphorbiaceae } & 14 & 31 \\ \text { Gramineae } & 15 & 25 \\ \text { Rubiaceae } & 13 & 17 \\ \text { Solanaceae } & 11 & 23 \\ \text { Verbenaceae } & 11 & 19 \\ \text { Bromeliaceae } & 11 & 15\end{array}$

Members of the Leguminosae are the most common plunts. Everywhere these shrubs and trees with their often numerous small leaflets are to be found. These have also some of the most striking flowers, as the Poinciana, the Caesalpinias, Tamarindus, the Cassias, and Calliandra.

The paucity of the Compositae, on the other hand, and the inconspicuous character of their flowers are remarkable when compared with the traits of the group in other regions, either tropical or temperate. In Margarita the family is chiefly represented by insignificant weeds.

The lack of grasses and sedges, of course, is very striking to a traveler from the temperate regions. There is no such thing as turf and the few grasses that are to be found are rather scattered.

One of the families that is largely represented in individuals if not in species is the Cactaceae, a group which corers the plains for miles. On Margarita there are twelve species of this family distributed in six genera. Of these, five are more or less tall and candelabrashaped, with little or no secondary branching; two are melon-shaped, one (Pereskia) is a low much-branched tree, and one (Rhipsalis) is a small cord-like epiphyte.

Another family, that of the Bromeliaceae, is characteristic not because of its few individuals but because of their striking colors and general appearance. All of the representatives of the eleven genera and fifteen species possess a rosette habit and more or less fleshy leaves. Their colors, too, are attractive. 
Other groups characteristic of tropical regions and to be found on Margarita are the Melastomaceae, Aroideae, Piperaceae, Loranthaceae, and such members of the Filices as Trichomanes, Hymenophyllum, and Cyathea.

Thus it may readily be seen that the variety of plants is rather great. One finds all conditions from that in which the halogens and other xerophytes of the seashore and plains are found to that of the mesophytes in the moist mountain regions, and not alone terrestial plants but also many epiphytes and a few phanerogamic parasites.

\section{Flora of the Islani of Coche.}

\section{Description of the Vegetative Conditions.}

The island of Coche is a typical desert island and, so far as reported, had never been visited by a botanist. The conditions existing on such an island were of great interest to me, and the fact that two new species of plants were found together with the fact that few descriptions of such an island are on record, makes it seem desirable to describe the flora of Coche and the regetative conditions in such detail as may be possible.

It was through the kindness of friends at Porlamar, Margarita, that I was enabled with a companion, Dr. A. F. Blakeslee, to visit Coche. The party, in charge of Sr. Antonio and Sr. Guilarte, left Porlamar at noon, Aug. 4, 1903, in a sloop, and reached San Pelro on the western end of Coche, shortly after nightfall. Although the sign over one doorway proclaimed "Restaurant" within, yct the proprietor refused to furnish either food or shelter. Fortunately, our companions had friends there so that rooms in different houses were assigned where hammocks, which had been brought along, could be slung. The meals were picked up as well as could be done at the stores. Drinking water cost two and one-half cents a liter. It was found that this water was brought over from Porlamar and from the mainland. Water was to be had from pits on the shore but it was undesirable for drinking purposes.

On the morning of the 5th of August Dr. Blakeslee and I traversed the low shore of this end from the south point to the north. The beach stretches for a kilometer and a half to the northwest into a narrow spit of land. On this extent of lowland is a salt lake, where great piles of salt are gathered for shipment. Beyond the lake itself for another 
kilometer the beach extends eastward, still a dazzling white. In one portion of the lake, the bottom is covered nith a pink animal growth while the rest is a clear blue. The presence of quicksands prevented any close examination of the lake.

Inland from the beach on this end the hills rise gradually and are rounded. In general, the hilltops are hard, corered with loose stone and sand. A few hilltops are entirely without soil, merely a mass of loose broken fragments of rock with no vegetation. The surface inland is rolling, there being no level surface of any extent. From the hilltops to the hollows or small valleys, the surface gradually gets sandier until at the bottom it is a clear stretch of sand free from any stones.

The seashore on the southwest side presents a contrast to that on the west. The beach is about a meter in width for three kilometers or more, and gravel and stony cliffs rise abruptly to the height of twentyfive or thirty meters. From the top of the cliffs the inland surface does not rise over thirty meters higher. About three kilometers from San Pedro the cliffs are lower, and here show abundance of hard rock. The lower part of the cliff and also huge boulders broken off are smoothly worn and dark red or brown in color. At the eastern extremity of the south shore, the beach stretches to the southwest in a long narrow spit of land.

The total length of the island is about twelve kilometers, and breadth three kilometers. From alınost any of its hills can be seen Margarita, at the nearest point within eight kilometers; and to the south the high mountain ranges show distinctly on the mainland about twenty-four kiloneters away. The village of San Pedro is composed entirely of homes of ordinary fishermen and of the pearl fishers. There is a church, a graveyard, and serenty-five to a hundred houses besides several stores. The eastern extremity of the island is used for the pearlfishing business but there is no village there.

No mention of rain on the island has been made because the existence of any at any time was not apparent. The natives claimed there never was any. As a matter of fact at the time of this visit there was a slight mist in the afternoon during the passing of a heavy thunderstorm on the coast of the mainland. This mist was barely perceptible and of course not sufficient to furnish water in any quantity. No streams or springs exist on the island. The unweathered condition of the rocks suggests the great lack of rain, while the presence of the sand 
in the hollows rather than on the hilltops may be entirely accounted for by the wind movements. The rounded hills are wind worn, and there are no gullies due to rushing water. It is noticeable that it is the northern shore that has the gradual slope, and the southern that is precipitous. This of course is due to the fact that the prevalent wind is from the northeast rather than from the south.

As to the vegetation that occurs on such an island it is either adapted to a life on the salt seashore or suited to withstand extreme drought inland. The land spit on the eastern end is bordered on the inside shore by mangroves. The beach about the "salina" or salt lake produces the customary Batis, Salicornia, Trianthema, and Alternanthera. On the inland side of the salt plain the bushes of Castela are abundant. Cassia hispidula is scattered here also. Pavonia cochensis is abundant in the sandy valley back of San Pedro. The valleys are characterized by the low Pereskia trees, the Acacias, and Gomphia, Guaiacum, Pithecolobium, and the largest of the trees, Bumelia cumeata, about three meters high. The smaller plants here are Jatropha gossypifolia, Solanum polyacanthos in the valleys; Croton, Argithamnia, Opuntia, Melocactus, and Waltheria on the rocky hillsides or hilltops; and in the most rocky as well as in sandy places Stylosanthes viscosa, the most abundant plant on the island.

Of all the foregoing plants only one, Argithamnia cochensis, is exceptional in color. This is dark red throughout. The other plants are gray at least as to the trunk and stem, and the leaves are a graygreen and either protected by dense pubescence or are leathery.

There appeared to be no plants of economic importance on the island. A few straggling palms are in the village of San Pedro, but they appeared to be neither in flower nor in fruit.

At the time of our visit, the only plants in bloom were Stylosanthes, Caesalpinia, and Bumelia. On Gomphia many dried remnants of fruits still remained. 'This, together with the fact that several trees showed signs of budding, suggests that at another tine more flowers and possibly more plants might be found. As a matter of fact no annuals were collected, but all were plants with extremely long and woody roots. Some specimens of Stylosanthes which were but $8 \mathrm{~cm}$. in height above ground had roots $22 \mathrm{~cm}$. long.

This collection while meager in its specimens probably represents well the flora of the island. The result may be summarized as follows:- 


$\begin{array}{lr}\text { Species of Plants } & 37 \\ \text { Genera } & 33 \\ \text { Families } & 21 \\ \text { Endemic species } & 2\end{array}$

Following is a list of the plants of Coche, those numbered being collected and the others merely noted. Citations are given for those plants which I found only on Coche, citations for the others being referred to the list of Margaritan plants.

Catalogue of Plants.

GRAMINEAE.

Sporobolus virginicus (L.) Kunth.- On Margarita alsu.

BROMIELIACEAE.

Tillandsia recurvata L.- No. 14. On Margarita alsu.

T. utriculata L. -No. 15. On Margarita also.

\section{LORAN'THACEAE.}

Phoradexdrox rubrum (L.) Nutt., Joum. acad. Phila., n. s., vol. 1, p. 185 (1S4S); Griseb., Fl. Brit. W. Ind., p. 314 . Viscum rubrum L., Sp. pl., vol. 2, p. 1023 (1753).-No. 10. Distribution general in tropical America.

\section{CHENOPODIACEAE.}

Atriplex cristata II. \& B.-On Margarita also.

SAlicorita fruticosa L.- No. 9. On Margarita also.

\section{A.IARANTHACEAE.}

Alternanthera Canescens HBK.-On Margarita also.

Gomphrent pilosa (Mart. \& Gal.) Moq.-No. 13. On Marga. rita also.

\section{BATIDACEAE.}

Batis Maritina L.- No. 16. On Margarita also.

AIZOACEAE.

'I'RIANTHEMA PORTUlaCastrum L.- On Margarita also. 


\section{CAPPARIDACEAE.}

CAPPaRis SP.- No. 1.

Capparis stenosepala Urb.- No. 2. On Margarita also.

\section{LEGUMINOSAE.}

Caesalpinia coriaria (Jacq.) Willd.-No. 3. On Margarita also.

Cassia hispidula Vahl, Eclog. Am., vol. 3, p. 10 (1807); Mart., Fl. Bras., vol. 15, pt. 2, p. 131.- No. 4. Further distribution, Mexico.

Pituecolobium unguis-cati (L.) Benth.- No. 11. A tree, $3 \mathrm{~m}$. high, top wide-spreading. On Margarita also.

Stylosanthes viscosa Sw.- No. 6. On Margarita also.

Zornia Bracteata (Walt.) Gmel., Syst., p. 1096 (1791).- Anonymos bracteata Walt., Fl. Car., p. 181 (1788).- No. 5. Further distribution, North America and South Africa.

\section{ZYGOPHYLLACEAE.}

Guajacum officinale L.-No. 19. On Margarita also.

\section{SIMARUBACEAE.}

Castela Nicholsoni Hook.-On Margarita also.

\section{EUPHORBIACEAE.}

Argitimamin cochensis Johnston, Proc. Amer. acad. arts and sci., vol. 40, p. $6 S S$ (1905).- No. 12. Endemic.

Croton flavens L.- No. 7. On Margarita also.

Jatropha gossypifolia L.- On Margarita also.

J. urens, var. stinulosa Muell. Arg.--On Margarita also.

\section{MALYACEAE.}

Pavonia Cochensis Johnston, Proc. Amer. acad. arts and sci., vol. 40, p. 690 (1905).- No. 1S. Endemic.

STERCULIACEAE.

Waltheria americana L.- No. 24. On Margarita also. 


\section{OCHNACEAE.}

Gonphia PYRIfolia Griseb., Fl. Brit. W. Ind., p. 105 (1859).- No. 20. A tree, about $3 \mathrm{~m}$. high.

\section{TURNERACEAE.}

Turnera diffusa Willd.- No. 21. On Margarita also.

\section{CAC'TACEAE.}

Cereus iargaritensis Johnston.-- On Margarita also.

C. eburneus Salm-Dyck.-On Margarita also.

Melocactus communis Link \& Otto.- No. 22. On Margarita.

Opuntia Tuna Mill.-- On Margarita also.

Pereskia opuntiaeflora DC.- On Margarita also.

\section{RHIZOPHORACEAE.}

Rhizophora Mangle L.- On Margarita also.

\section{MYRSINACEAE.}

JACQUinia Berterir Spreng., Syst., vol. 1, p. 668 (1825) - Further distribution, San Domingo. A tree, about $3 \mathrm{~m}$. high.

\section{SAPOTACEAE.}

Bumelia cuneata Sw.- On Margarita also.

\section{SOLANACEAE.}

Lycium salsum Ruiz. \& Pav.- No. 8. On Margarita also.

Solanum polyacanthos Lam., Illust., vol. 2, p. 23 (1793).-No. 17.

Comparison of the Flora of Margarita and Coche with that of Other Regions.

Two different points of view may be taken in comparing the floras of various regions: one consists of a study of the lists of plants collected from the regions in question, and the other consists of a study of the types of regetation. In using the former method one need only 
resort to the collection of plants in the various herbariums and to the lists of plants published in various books and journals; in this way the distribution of the plants is discovered, the knowledge of which is valuable both to the taxonomist and the biologist. In this method the research worker must either for himself consult all the plants in the herbariums and represented in the lists and must verify their identification or else depend for their value upon the person identifying the plant or publishing its name. It is not necessary to see the plant growing. The species are all units and are well represented as such by mere names.

In using the second method of comparative study, that of considering types of vegetation, one encounters a very different proposition. Types represent groups of plants and groups which do not necessarily consist of separate species, in fact a type of vegetation may be made up of a number of species. Types of vegetation, then, can be represented only very indefinitely by published lists of names of plants or by the plants themselves as they occur mounted on herbarium sheets. It is true that names are in use to designate certain types of regetation, terms such as xerophyte, hydrophyte, mesophyte, and others of more restricted meaning; and it is certain that these terms picture to one at all familiar with such work definite features in the regetation. Moreover, the characteristics of any type of vegetation are often so well shown in the species constituting that type that one can by examining herbarium'specimens very commonly determine to what type of vegetation the plant in question belongs.

So far it may be seen that one can by research among collections and lists of plants obtain both a fair idea of the species of plants from any particular region and some knowledge of the type or types of vegetation found in that region. So much can be done at home. The types, however, represent groups, and as the groups are often composed of many species which have one feature in common but often have a wide range in size, outline, method of branching, and color, it is impossible to give a complete and at all real picture of a locality without considering these various features, characteristics which can be ascertained only by traveling among the regions in question and by viewing personally the types of groups or composites of species as they are growing together in the various localities. The modifications of gromps are so great that it is necessary to visit many localities and to visit the same localities at various times of the year before one can obtain a true knowledge of the character of the vegetation. 
In order then to make an intelligent and thorough comparison of the flora of Margarita and Coche with that of other regions about the Caribbean Sea, it would be necessary for one to have access to specimens of plants and lists of plants collected in all lands bordering the Caribbean and also to have traveled in those regions. So far as the lists of plants and the collections are concerned it must be said that despite the many visits of American botanists to the West Indies and tropical America, and despite their intense activity in the United States, there is yet very much to be known about the plants in those regions. Parts of Mexico and of Central America are being well worked over. Colombia and Venezuela have had so little work done on them that comparison of lists of their plants is almost valueless. The West Indies as a whole have the useful works of Grisebach and of Professor Urban but these contain no lists of plants of the individual islands, so that they are scarcely to be used in comparative work. A few of the American botanists frequent Mexico and parts of Central America, a very few have visited Colombia, still fewer Venezuela, and some frequent Cuba, Hayti, Porto Rico, and Jamaica. Seldom is the traveling extensive or in more than one region. Both plant and animal surveys of the United States are fairly thorough through many parts, but in tropical America and the west Indies, biologically closely related to our southern States, little such systematic work has been done.

In view of the situation as above discussed it is perhaps particularly desirable that I make such a complete comparison of the flora of Margarita and of Coche as may be possible with that of other regions, especially considering that I have what I believe to be a complete list of all plants ever collected in or recorded from Venezuela and also that I have been enabled to visit personally many parts of Venezuela, British Guiana, and Panama, and many of the West Indian islands.

In a preceding chapter on the composition of the flora of Margarita, it would seem that the vegetation of Margarita partook equally of the nature of the West Indian and of the South American elements. This appearance I believe to be due to the large proportion of cosmopolitan plants present. In reality Margarita is, as would be expected, distinctly South American in its flora as will appear in the following pages.

The two islands under discussion, Margarita and Coche, are the 
most eastern of all those along the northern coast of Venezuela. The other islands to be considered are Testigos, Blanquilla, Orchilla, Los Aves, Los Roques, Cubagua, Tortuga, Aruba, Buen Ayre, and Curaçoa, the respective positions of which may be seen by referring to the accompanying map of the Carribean Sea and its surrounding lands (Pl. 23). All of these islands are identical in topographical features with the plains and hills of Margarita.

Of this group Cubagua is eight and one half kilometers (5.25 mi.) from Margarita and twice the distance from Coche, of which it is almost a counterpart in size and physical characteristics. No botanical research has been made upon it, though I have passed very near the shore sereral times and have observed that it has the same barren features that Coche possesses. There is no water on the island and never has been any within historical time, and though in the early sixteenth century it was the site of a thriving city of Spanish pearlfishers, at present there may be seen only a few fishers' huts.

The next nearest island is La Tortuga, ninety kilometers (60 mi.) from Margarita and the same distance from the coast of Venezuela. The island is twenty kilometers (12 mi.) from east to west and ten kilometers $(6 \mathrm{mi}$.) from worth to south. It is merely a raised coral reef and presents the appearance of a low waste of land with an almost level surface and a very narrow beach. The regetation as reported by Ernst consists of sixty-nine different species of plants largely common to American tropics of which, however, twenty-three are not to be found on Margarita.

The islands Los Aves, Testigos, and Blanquilla, so far as is known, have never been visited by a botanist. Los Aves consists of a number of small rocky and barren islets midway between Buen Ayre and Los Roques. 'They have been noted solely as a source of guano. The vegetation would naturally be very scanty. Testigos is a small group eighty kilometers (50 mi.) north of Margarita; and Blanquilla which is somewhat larger, being twenty-five kilometers in circumference, is seventy kilometers north of Margarita. Though there are no recorded visits to these islands, the flora may be assumed to be very scanty and to consist for the most part of cosmopolitin seashore plants.

Los Roques is a group of islands one hundred and thirty kilometers (80 mi.) from the coast of Venezuela and consists of a dozen or more rocky islands none over a kilometer in length. The flora is entirely 
that of a tropical seashore, and is similar to that of Tortuga and Coche and the coast of Margarita, though more limited. As reported by Ernst there are twenty-four different species, of which Cyperus brunneus, Cyperus caesius, Eragrostis prolifera, and Opuntia spinosissima have not been reported from either Margarita or Coche. 'Two of these, Cyperus caesius and Opuntia spinosissima, are not found on Tortuga. It will be noticed, however, that these are of wide distribution.

The three western islands lying off the coast of Venezuela belong to the Dutch Republic. The most important work that has been done upon them botanically was by Suringar ('86). He lists, however, scarcely a dozen plants from Aruba and Buen Ayre. From the character of these two islands one is led to expect a flora similar to that of Curaçoa though more limited. Buen Ayre is about forty kilometers (25 mi.) east of Curaçoa and has an area of twenty-four thousand hectares (85 sq. mi.), while Aruba is nearly seventy kilometers west of Curaçoa with an area of seventeen thousand hectares (69 sq. mi.).

Curaçoa is the one of this group upon which some botanical work has been done. Several botanists have visited the islands, the most notable of whom was perhaps Suringar. As determined from his list of Curaçoan plants (117 species), fifty have not been reported from Margarita. To be sure Suringar's list is not taken to be at all complete of the flora of Curaçoa. Simons ('68) has published a much longer list but unfortunately the names are in a great part old ones and quite impossible to identify for certain with those at present in use. Even considering this list, Margarita has over four hundred plants not on Curaçoa. This difference in the flora is due to marked contrast in physical conditions and to their difference in geographical position. In physical condition Curaçoa resembles the plains and hills of Margarita, and has a vegetation similar to theirs, but the heavy woods and the varied vegetation of the mountain tops are to be found in Margarita only. In geographical position, although they are both coastal islands and are on nearly the same parallel, yet they are five hundred kilometers (300 mi.) apart and Curaçoa is ninety kilometers (50 mi.) from the coast of Venezuela. It is probable that complete exploration of both islands would show more resemblances between them, but according to present knowledge, the differences in physical conditions and in geographical situation alone may well account for the differences in the floras. 
All of these islands extending along the north coast of Venezuela not only resemble each other very much but they are also like the coast of the mainland. There are to be found much the same species constituting the seashore flora, the flora of the lagoon, of the wild cactus-covered hills, and of the few fertile coconut valleys. This is well illustrated by almost any part of the north coast. Carúpano is in a long narrow valley with arid hills on each side. Cumaná is on a sandy plain at the foot of the hills. Guanta is in a small valley with the appearance of a perpetual drought on every side. La Guaira is on a hillside by the edge of the sea and the hill is a brown and sunbaked exposure although it is broken here and there by green valleys and by a green mountain rising above. A short way inland but still in the coastal region between Caracas and Valencia and about the Lake of Valencia trees are scarce or lacking, the mountains are brown and clothed only in small shrubs or in dry grass, and in the valley are scorching sandy plains with here and there the shade of a tree.

'These islands are similar to the coastal land as naturally they should be, having been in early times a part of the coast and yet there is a vast country behind the coast to which they are not at all like. The mountain region of the Andes, anywhere from one thousand to four thousand meters high, the grassy plains of the Orinoco, and the forests to the south present features vastly different in erery respect. Unfortunately our knowledge of their flora is rery limited. Many plants were described as new from Humboldt's travels, but since that time there have been few collections and fewer plants described. It is known that there are many plants which are common to the rest of the tropics. Altogether our information is one-sided as tending to show the cosmopolitan rather than the characteristic plants. I have compiled a list of all the published names of Venezuelan plants which comprises some three thousand names. That some of these are names which may not be in good standing today cannot be denied, but I have at least made reasonably sure that they represent nearly three thousand different species.

Out of the six hundred and thirty-four Margaritan plants two hundred and ninety-five have not been published as occurring anywhere else in Venezuela. Inasmuch as many of these are cosmopolitan plants it shows not the peculiarity of the Margaritan flora but the small amount of work that has been done on the mainland. 
Close to the mainland but yet not considered one of the Venezuelan Islands is 'Trinidad lying to the northeast of Venezuela. In general the flora and regetative conditions of the island are similar to those of the mainland. There is a large collection of well identified plants at the St. Clair experiment station at Port-of-Spain and I had the opportunity of comparing my first collection with them. From this I found that one hundred and serenty-nine Margaritan plants were not in the Trinidad herbarium and one hundred and sixty-one were there. Unfortunately it has been impossible for me to compare the remainder of my plants. Of those plants that were not in the Herbarium the majority were of wide distribution. Of the plants of restricted distribution, however, the greater number were Venezuelan rather than pertaining to the West Indies.

Trinidad presents a distinct contrast in the appearance of its regetation to that of the islands of the north coast. The latter are dry as is the adjacent coast. Trinidad, however, resembles and is really a part of the east coast, characterized. by low land, well watered, and heavily wooded. Thus appears the east coast of Venezuela and of British Guiana, green with luxuriant regetation, broken here and there, to be sure, with sandy stretches and low hills, but in effect with a truly tropical verdure. Trinidad has a series of hills across the low end of the island and a range of low mountains across the northern end. The regions about these hills are in many places heavily covered with forests. Across the middle of the island is a belt of almost unbroken savannah land much used for grazing and for cane crops. Both on the eastern and on the western side are extensive swamp lands. Only in isolated and restricted areas are there any arid districts at all resembling those of Margarita.

The entire chain of small islands extending from Trinidad northward is with few and unimportant exceptions similar in regetative conditions to Trinidad. For the most part they consist of well cultivated plains and green-clad hills and mountains. Porto Rico at the northern end of this chain of islands and the easternmost of the Great Antilles presents features somewhat different. With the exception of a narrow plain about the island, the surface of Porto Rico is undulating and broken into sharp hills and ridges from one end to the other. In marked contrast to Trinidad, Porto Rico has very little forest land and only a few small savannahs. Moreover, the waste lands, barren or cactus-covered, along the south shore are comparable 
with the wastes of Margarita. This northern island is perhaps in a condition midway between that of the coast and coastal islands of Tenezuela and that of Trinidad, the former being in a condition of drought and the latter in a state of excessive moisture for much of the year. In regard to the species of plants, there is of course a vast difference. Professor Urban's Flora Portoricensis is very complete so far as published. In it are sixty-one plants to be found on Margarita, although the author does not refer more than twenty-five to the island, these references being only from my first collection of plants. As many as this is naturally to be expected from the wide distribution of many of the plants. Moreover, there are fully five hundred plants of Margarita not to be found on Porto Rico, and of course many more on the much larger island not on Margarita.

In the small Cayman Islands farther west in the region of the Great Antilles there is also a diversity from Margarita. These islands consist of Grand Cayman, Little Cayman, and Cayman Brac, two hundred and eighty-nine kilometers (180 mi.) northwest of Jamaica and about the same distance south of the center of Cuba. Grand Cayman is twenty-seven kilometers from east to west, six to eight wide at the eastern end and eleven to thirteen kilometers ( 7 to $S$ mi.) wide at the western end. There is no elevation exceeding fifty meters (150 ft.). Some forest land is present, and in the center is considerable boggy soil suggesting the presence of sufficient moisture for much vegetative growth. Collections of plants have been made on the Caymans by Professor C. F. Millspaugh and by Mr. W. Fawcett. From the total of two hundred and twenty-eight species constituting these lists eightyfour are found on Margarita. Five hundred and eighty Margaritan plants are not found on the Caymans. This suggests a distinctly different flora notwithstanding the presence of so many plants of wide distribution. This difference can be accounted for partially in the vegetative conditions, but in the main it is due to geographical position, the Cayman Islands being some seventeen hundred kilometers northwest of Margarita. Moreover in comparing the flora of the Cayman with that of the other Venezuelan islands or with Trinidad the same result is obtained.

It is impossible to make a definite comparison of the plants of Jamaica and Cuba with those of Margarita, for the lists are so incomplete. It must suffice to say that from the material available for comparison it is certain that a very large part of the plants of Jamaica and 
Cuba is not to be found in northern Venezuela excepting of course the flora common to all tropical countries. The vegetative conditions of these islands can be said to differ from portions only of Venezuela. The waste plain west of Kingston is duplicated in the plain of Margarita. The valleys and ridge of the Blue Mountains resemble those of the coast range above Caracas in Venezuela. The barren mountains about Santiago de Cuba are identical in appearance with range after range extending from Caracas to Valencia. The rolling land that occupies the most of Cuba with its cane fields and tobacco fields is similar to that of the interior of Venezuela. There still remains in Cuba some of the virgin forest. In fact there is considerable of it and it suggests that of the more nearly equatorial countries. Only in a limited way, however, does it begin to compare with a truly tropical forest. Such regetation as is to be found along the shores of the Orinoco, Essequibo, and the Amazon is nowhere to be found among the northern islands.

Further north than the islands of Jamaica and Cuba, in subtropical Florida there are naturally many changes from the tropics. Some districts approach in their wild luxuriance of regetation that of countries near the equator and on the other hand there are to be found some stretches barren as the desert regions of the Venezuelan islands. As a whole, however, the individual species making up the type of vegetation have changed. The difference in temperature of the regions sets a limit upon the distribution of the species. In passing, it is of interest to note that notwithstanding the great differences in species, exclusive of cultivated plants there are ninety-seven different species occurring in both southern Florida and Margarita.

\section{Conclusion.}

In writing the foregoing pages three objects have been foremost in my mind: to catalogue the plants of Margarita, to describe its vegetative conditions, and to compare its flora with that of adjacent regions.

The catalogue of the species comprises 644 names, all of the plants that have been reported from Margarita. Collections, however, have been made only on part of the eastern end and during only a part of the year (March, July, August, and the first four days in September) thus leaving opportunity for much additional work. It is doubtful if this catalogue comprises much more than three fourths of the entire 
flora of the island. Forty-two new species, including two new genera, have been discovered on the island.

'The vegetative conditions of Margarita are much more varied than those of the other islands. Margarita has both a rich mountain flora and also the flora of arid plains and hills. Curaçoa and the others possess only arid regetative conditions.

In regard to the comparison of the flora with that of adjacent regions, it is much to be regretted that data are so insufficient as to lessen the value of any comparison and in some cases actually to prohibit it. 'The flora of Margarita comprises all the plants found on Coche with three exceptions. 'The other small islands are probably similar in this respect. La Tortuga has twenty-three out of sixty-nine plants not to be found on Margarita and Los Roques has four out of twenty-eight not on Margarita. Though it is impossible to speak accurately of Curaçoa, to judge by the references cited on previous pages there are about four hundred plants there of which one hundred are not on Margarita.

Although there is a large list (240) of plants of Margarita not published as occurring in Venezuela, it is probable that a large proportion of them do. The regetation on the mainland (near Carúpano and Cumaná) opposite Margarita is identical in appearance with that of Margarita.

Trinidad has a very large flora, yet over two hundred Margaritan plants have not been reported from there, and are not in the Herbarium of the Trinidad botanical gardens.

The entire chain of islands to the east of the Caribbean Sea possesses a vegetation consisting of many species not to be found on Margarita. It is of a much more luxuriant character. In the extensive flora of Porto Rico so far as can be ascertained there are less than one hundred Margaritan plants to be found. Most of these are common to the American tropics.

In the flora of the Cayman islands it is seen that out of their two hundred and twenty-eight species only eighty-four are on Margarita. The reference to the plants of the southern United States similarly shows about a hundred from Margarita which are, however, cosmopolitan.

In the comparison of the flora with that of other regions about the Caribbean Sea it is evident that the flora of Margarita is largely composed of plants common to many parts of the American tropics. It 
is also seen in studying the distribution of the individual plants that Margarita contains twice as many plants which are characteristic of South America as are characteristic of the West Indies. And finally, it is still as clearly evident from comparing it with other islands about the Caribbean Sea that while Margarita has some plants common to all of the islands, yet as a whole it has a flora quite distinct from the northern islands and at the same time closely approaching that of the Venezuelan islands and the north coast of the mainland. 


\section{LITERATURE.}

To show the more important publications on the exploration, botanical collecting, and geography of Venezuela, the following bibliography is added.

\section{Geography of Venezuela.}

Note.-The following are selected from a list of about fifty books on Venezuela, and include, it is believed, all that are of any value to the botanical worker.

\section{Anonymous.}

'24. Letters written from Colombia during a journey from Caracas to Bogotá. G. Corvie \& Co.: London, 1824, 208 pp., map.

Gives description of the route as to forests, meadows and cliffs, as to agriculture, towns, etc.

'73. Memoria de la Direccion general de estadistica al Presidento de los Estados Unidos de Venezuela en 1873. Impr. Nacional: Caracas, 1873,3 vols.

Very complete as to geography and cornmerce.

André, Eugéne.

'04. A naturalist in the Guianas. Scribner's Sons: New York, 1904, 310 pp., 32 pls., map.

André went on two expeditions up the Orinoco and Caura Rivers.

Bénard, Charles.

'97. Le Vénézuéla. Impr. G. Gounouilhou: Bordeaux, 1897, 106 pp., map.

Gives heights of mountains, length of rivers and drainage area, table of temperatures, agriculture, and animals.

Caulin, Antonio.

1779. Historio coro-graphica natural y evangelica de la Nueva Andalucia, prov. de Cumaná, Guayana y vertientes del rio Orinoco. J De San Martin: Madrid 1779, 482 pp., 3 pls., map.

A natural history, including descriptions of the rivers.

Cazeneuve, Paul de and François.

'88. Les États-Unis de Vénézuéla. Sauvaitre: Paris, 1888, 300 pp., map.

A good map, a very good guide containing population of many cities, courses and lengths of many rivers, medicinal plants, and history. Codazzi, Agustin.

'41. Resúmen de la geografia de Venezuela. Impr. de H. Fournier y Compia: Paris, 1841, 648 pp.

The most complete geographical work on Venezuela. 
Dauxion-Lavaysse, Jean François.

'20. A statistical, commercial, and political description of Venezuela, Trinidad, Margarita, and Tobago. G. \& W. B. Whittaker: London, $1820,479 \mathrm{pp}$.

Contains an interesting sketch of Margarita.

Duane, William.

'26. A visit to Colombia in the years 1822 and 1823 . Venezuela, p. 1-351. T. H. Palmer: Philadelphia, 1S26, $632 \mathrm{pp.}$

Gives a good description of the hills and the valleys, and the wooded and the barren regions between Caracas and Colombia.

Engel, Franz.

'88. Auf der Sierra Neváda de Merida. A. G. Richter: Hamburg, $1888,36 \mathrm{pp}$. Sammlung gemein verständlicher wissenshaftlicher Vorträge, n. f., series 3 , no. 58 .

Ernst, Adolphus.

'68-'69. Ascension á la Sierra Neváda de Merida, Feb. 18, 1868. Vargasia. Caracas, $1868-69$, p. 199-202.

'84-'86. La Exposicion Nacional de Venezuela en 1883. Impr. de la Opinión Nacional: Caracas, 1881-86, 2 vols., map.

Gerstächer, Friedrich.

'68-'69. Neue Reisen durch die Vereinigten Staaten, Mexico, Ecuador,

West-indien und Venezuela. H. Castenoble: Jena, 1868-69, 3 vols.

Chapters on La Guaira, Caracas, Valley of Aragua, llanos, San Fernando de Apure, Apure River, Orinoco River, and mines at Angostura.

Goering, Anton.

'93. Vom tropischen Tieflande zum ewigen Schnee. Eine malerische Schilderung des schönsten Tropenlandes. A. Fischer: Leipzig, 1893, 54 text figs., 12 colored pls.

Though rough sketches, the illustrations of this work give some idea of Maracaibo, Merida, and Mucuchies.

Humboldt, Alexander, and Bonpland, Aimé.

'14-'29. Personal narrative of travels to the equinoctial region of the new continent, during the years 1799-1804, translated by Helen M. Williams. Longman, Hurst, Rees, Orme, and Brown: London, 1814-29, 7 vols.

Contains descriptions of the country about Cumaná, Cumanacoa, and San Fernando de Apure.

'14. Voyage aux régions équinoxiales du nouveau continent. F. Schoell: Paris, 1814, 3 vols., 2 atlases.

Contains full description of route.

Kol, H. van.

'04. Naar de Antillen en Venezuela. A. W. Sijthoff: Leiden, 1904, $552 \mathrm{pp}$.

Description of Curaçoa. Good illustrations of islands. 
Landaeta Rosales, Manuel.

'89. Gran recopilacion geografica, estadistica, é historica de Venezuela. Impr. Bolivar: Carácas, 1889.

Lennep Coster, G. van.

'42. Aanteekeningen, gehouden gedurende mijn verblijf in de West Indiën in de jaren 1837-40. J. F. Schleijer: Amsterdam, 1842, 359 pp. Chapters on Curaçoa, Aruba, Bonaire, and Venezuela.

Lével, André Aurelio.

'81. Esbozos de Venzuela I. Margarita. Caraćas, 1881, p. 26-50.

Morisse, Lucienne.

'04. Excursion dans l'Eldorado (El Callao). Association d'imprimeurs: Paris, 1904, 474 pp., 4 maps, diagram.

Only popular work treating of the country between Ciudad Bolivar and British Guiana. Pertains particularly to the mining regions.

Ober, Fred A.

'91. The Knockabout club on the Spanish Main. Estes and Lauriat: Boston, 1891, 239 pp.

A story incidentally giving much information valuable to a traveler new to the country.

Paez, Ramon.

'68. Travels and adventures in South and Central America. C. Scribner \& Co.: New York, 1S68, 473 pp., map.

Description of llanos and life of llaneros.

Pocaterra, Jaime D.

'64. Derrotero del Golfo de Venezuela ó soco de Maracaibo. Impr. de S. Hallet: New York, 1864, $32 \mathrm{pp}$.

Description of shores, winds, and currents of Lake Maracaibo.

Robinson, J. H.

'22. Journal of an expedition 1400 miles up the Orinoco and 300 up the Arauca. Black, Young, and Young: London, 1S22, $397 \mathrm{pp}$.

Of little value so far as descriptions are concerned.

Roncayolo, L.

'94. Au Venezuela, 1876-1892. P. Dupont: Paris, 1891, 208 pp. Excellent photographs, chapter on Merida, etc.

Sievers, Wilhelm.

'88a. Die Cordillere von Mérida nebst Bemerkungen über das karibische Gebirge. Geogr. Abhand., vol. 3, no. 1. Hölzel: Wien, 1888, 238 pp., geolog. chart.

Good chapters on rivers, lakes, climate, vegetation, agriculture, etc. Bibliography especially good on geology.

'88b. Venezuela. L. Friederichsen \& Co.: Hamburg, 18ss, 359 pp., map. 
Full description of paramo region, of alpine or mountain regions in general, and of llanos. Good geography of T'enezuela.

'96. Zweite Reise in Venezuela in den Jahren 1892-93. L. Friederichsen \& Co.: Hamburg, 1896, 327 pp., map.

Contains a good map of coast and islands from Coro to Trinidad.

\section{MaPS.}

The maps in IV. Sievers's works noted above are of special value. In addition may be noted the following:-

'88. Texte et carte commerciale des États-Unis de Vénézuéla avec notice descriptive Imprimerie Chaix: Paris, 1888.

97-'98. Orinoco-Essequibo Region. Map 2. Senate doc., vol. 9, no. 91 , pt. $4,1897-98$.

There are also a number of coast charts issued by the U. S. hydrographic office.

\section{Vegetation of Venezuela.}

Anonymous.

'64. Indicacion de algunos de los principales objetos de historia natural, es decir: minerales, vegetales y animales - para la exhibicion 1864 . Imprenta de lo Estados Unidos de Venezuela, por Felix é Bigotte, Caracas, 1864, $22 \mathrm{pp}$.

'93. World's Columbian exposition at Chicago. The United States of Venezuela in 1893. Pub. by order of government of Venezuela. New York, 1893.

'04. International bureau of American republics. Tenezuela. Washington, D. C., 1904, 604 pp., map, illustr.

Excellent in every way.

Bellermann, F.

'94. Landschafts- und Vegetations-Bilder aus den Tropen Süd-Amerika's nach der Natur gezeichnet von Prof. F. Bellermann. R. Friedlander \& Sohn: Berlin, 1894, 24 pls.

Illust. of Cumana, La Guaira, Caracas, Merida, and vicinities. Text by H. Karsten.

Braun, A.

'58. Übersicht der Characeen aus Columbien und Guyana. Monatsber. königl. preuss. Akad. Wissensch. Berlin, 1858, p. 35t-367.

Caulin, Antonio.

1779. Historio coro-graphica. Madrid, 1779.

Chapters on the trees, fruits, medicinal plants, gums, resins, balsan, various animals, and rivers.

Dozy, F.

'54. Prodromus florae bryologicae Surinamensis. Accedit pugillus specierum novarum florae bryologicae Tenezuelanae. Arnz \& Co.: Düsseldorf, 1854, 54 pp., 19 pls. 
Eaton, Daniel C.

'61. Filices Wrightianae et Fendlerianae. Mem. Amer. acad. arts and sci., new ser., 1861, vol. 8, pt. 1, p. 192-220.

As an appendix to this is Orchideae Wrightianae et Fendlerianae.

Ernst, Adolphus.

'65a. On the medicinal plants of Caracas, Venezuela, and their Venezuelan names. Scemann's Journ. of bot., 1865, vol. 3, pp. 143-150, $277-284,306-322$.

201 plants of Venezuela mentioned.

'65b. Plants growing in the streets of Caracas. Seemann's Journ. of bot., 1865 , vol. 3 , p. 322-323.

'66a. Formas caracteristicas de la flora venezolana. Las palmas.

El Porvenir, Caracas, 1866, vol. 1, no. 6; vol. 2, no. 7, vol. 3, no. 8 .

'66b. List of Venezuelan woods, with their Venezuelan names and specific gravity. Seemann's Journ. of bot., 1866, vol. 4, p. 359-360.

Gives names of 48 plants, 24 of which are on Margarita.

'67a. On the plants common to the southern United States and Venezuela. Seemann's Journ. of bot., 1867, vol. 5, p. 290-296.

There are 2684 plants in Chapman's Flora; of these 283 are in Venezuela.

'67b. On the plants cultivated or naturalized in the valley of Caracas, and their vernacular names. Seemann's Journ. of bot., 1867, vol. 5, p. $261-275,287-290$.

'68-'69a. Los Helechos de la Flora Caracasana. Vargasia, Caracas, 1868-69, p. $100-103$.

'68-'69b. Plantas interesantes de la flora Caracasana. Vargasia, Caracas, $1868-69$, p. 178-194.

'72a. Verzeichniss der auf der venezuelanischen Inselgruppe Los Roques im Sept., 1871 beobachten Pflanzen. Bot. Zeit., 1872, vol. 30, p. 539$5+1$.

'72b. Sertulum Naiguatense; Notes on a small collection of alpine plants from the summit of Naiguatá, in the mountains of Caracas. Trim., Journ. of bot., Sept. 1872, vol. 10, p. 261-264.

'74. Observationes aliquot in plantas nonnullas rariores vel novas florae Caracasanae. Flora, 1874, vol. 57, p. 209-215.

Some of these plants were collected by Sr. Fermin Toro of Caracas; 24 plants mentioned, 6 of these new species of Ernst and 1 of Toro.

'76a. Descriptive catalogue of the Venezuelan department at the Philadelphia International exhibition, 1876. McCalla \& Stavely: Philadelplia, $1876,55 \mathrm{pp}$.

'76b. Florula Chelonesiaca. Trim., Journ. of bot., London, 1876, vol. 14 , p. $176-179$.

Thirty-one out of the 69 plants occur also on Margarita.

'77a. Estudios sobre la flora y fiuna de Venezuela. Imprenta Federal: Caracas, $1877,330 \mathrm{pp}$.

This work is also found in Primer anuario estadistico de Venezuela ano de 1877, Impr. Nacional: Caracas. Lists 412 orchids and 399 ferns and fern allies. 
'77b. Várgas consid. como bot., Caracas, 187\%. A paper presented to the society of physical and natural sciences in Caracas, 1877.

'79. Enumeracion de las plantas mas notables que fueron observadas en la excursion à Naiguatá. Repertorio Caraqueño, 1879, p. 141-146.

'86. Eine botanische Excursion auf der Insel Margarita. Nederl. kruidk. arch. Nijmegen, 1886, vol. 4.

'92. La vegetacion de los Páramos de los Andes T'enezolanos. Boletin del Ministerio de obras publicas, Caracas, 1892, no. 157, p. 159-163, Feb., March.

'00a. Sertulum Aturense, ó sea, lista de una pequeña coleccion den plantas que recojio el Sr. Alfredo John, hijo, en Octubre de 1887 cerca de Atures, Alto Orinoco. Revista cientif. de la Universidad Central deVenezuela, Caracas, 1900. vol. 1, p. 219-223.

'00b. Bibliographia (Prof. Dr. phil. A. Ernst, Caracas, Venezuela, 186599) Universitäts-Buchdrucherer, J. Neuenhalm: Jena, 1900 (?)

Goebel, $\mathrm{K}$.

'91. Die Vegetation der venezolanischen Paramos, in Pflanzenbiologische Schilderungen, 1889-93, pt. 2, no. 1. N. G. Elwert: Marburg, 1891.

Prof. Goebel visited many of the higher mountains about Merida and collected some plants.

Hampe, E.

'47a. Bericht über die Hepaticeae welche Hr. Moritz in Columbien sammelte und dem königlichen Herbarium in Schönberg überlieferte, nach der Synopsis Hepaticarum und den Moritzschen Nummern aufgeführt. Linnaea, 1847, vol. 20, p. 321-336.

Lists 48 plants of Venezuela.

'47b. Ein Referat über die columbischen Moose, welche von Herrn Moritz gesammelt worden. Linnaea, 1847, vol. 20, p. 65-9S.

Lists 70 plants of Venezuela.

Hegelmaier, Friedrich.

'68. Die Lemmaceen. Eine monographische Untersuchung. Wilhelm Engelmann: Leipzig, 1868, 169 pp., 16 pls.

Humboldt et Bonpland.

'08. Plantae aequinoctiales. F. Schoell: Paris, 1808, 2 rols., 140 pls.

Humboldt, Bonpland, et Kunth.

'15-'25. Nora genera et species plantarum. F. Schoell: Paris, 1815-25, 7

vols. Vol. 7 contains summaries entitled "Flora provinciarum Novae Andalusiae Venezuelae, nec non Planitiei Barcinonensis," and "Flora Orinoci et fluminis Nigri" the two containing 1170 Venezuelau plants. Johnston, J. R.

'05. New plants from the Islands of Margarita and Coche, Venezuela, Proc. Amer. acad. arts and sci., 1905, vol. 40, p. 683-698.

'08. Plants collected in the vicinity of La Guaira, Venezuela. Contrib. U. s. nat. herb., 1908, vol. 12, pt. 2, p. 105-111. 
Karsten, H.

'48. Auswahl neuer und blühender Gewächse Venezuelas. Berlin, 1848, 4 to, 12 pls.

'58-'69. Florae Columbiae terrarumque adjacentium specimina selecta, etc., $1858-69$.

Klotzsch, Joh. Friedr.

'47a. Beiträge zu einer Flora der Aequinoctial-Gegenden der neuen Welt. Linnaea, $18+4$, vol. 18 , pp. 515-556, 667-709; 1847, vol. 20, p. 337$432,446-512$.

'47b. Nachträge zu den Lycopodineis und Filicibus, welche im 18 Bandee der Linnaea p. 515-556 unter der Ueberschrift: "Beiträge zu einer Flora der Aequinoctial-Gegenden der neuen Welt" veröffentlicht wurden. Limnaea, 1847, vol. 20, p. 433-145.

Lists $3 \pm$ plants of Venezuela.

Kunth, Karl.

'39. Bemerkungen über die Familie der Piperaceen. Linnaea, 1839, vol. 13, p. $561-726$.

Landaeta Rosales, Manuel.

'89. Gran recopilacion geografica, estadistica, é historica de Venezuela. Impr. Bolivar: Caracas, 1889, 2 vols.

Vol. 1, p. 68-72, contains a list of Venezuelan plants under their vernacular names.

Loefling, Peter.

1776. Reise nach den spanischen Ländern in Europa und America in den

Jahren 1751 bis 1756. Berlin und Stralsund, 1766, 406 pp.

Maury, P.

'89. Enumération des plantes du Haut-Orénoque récóltées par MM. J. Chaffanjon et A. Gaillard. Journ. de botanique, Paris, 1889, vol. 3, pp. $129,157,196,209,260$, and 266.

Patouillard, N., and Gaillard, A.

'87. Champignons du Vénézuéla et principalement de la région du HautOrénoque, récoltés en 1887 par M. A. Gaillard. Bull. soc. mycol. de France, 1857, vol. 3, pp. 7-46, 92-129.

The fungi were collected about Caracas, Ciudad Bolivar, and San Fernando de Atabapo, 278 species in all.

Reichenbach, H. G., fil.

'54a. Die Wagener'schen Orchideen. Bonplandia, 1854, vol. 2, p. 9-26.

'54b. Orchideae Schlimianae. Bonplandia, 185t, vol. 2, p. 277-284.

The majority of Schlim's orchids were collected at Ocaña and Pamplona, Colombia.

'58-'00. Xenia orchidacea, Beiträge zur Kenntniss der Orchideen. Leipzig, 1858-1900, 3 vols. 
Rusby, H. H.

'96. Concerning exploration upon the Orinoco. Alum. journ. coll. pharm., N. I., 1896, vol. 3, p. 185-191.

Schiller, G. IV.

'57. Catalog der Orchideen-Sammlung von G. W. S. zu Ovelgönne an der Elbe. Hamburg. Ed. 3, 1857.

Seemann, Berthold.

'64-'67. Revision of the natural order Hederaceae. Seemann's Journ. of bot., vols. $2,3,4$, and 5 .

Contains a number of Venezuelan plants.

Simons, G. J.

'68. Beschrijving van het eiland Curaçou. G. S. Tempe: Oosterwalde, 1868, p. 156.

Suringar, IV. F. R.

'86. Nederlandsch-West-Indische expeditie, verslag en reisverhaal. Tijdschr. Nederl. aardrijskundig genootschap. Amsterdam, ser. 2., 18S6, pt. 3, afd. versl. en meded., pp. 45-90, 355-394, 511-545.

Villavicencio, $R$.

'80. La República de Venezuela, bajo el punto de la geografia y topografia medicas y dela demografia. A Rothe: Caracas, $18 s 0$.

Chapter on flora is excellent, describing the distribution.

Weddell, H. A.

'55. Chloris Andina essai d'une flore de la région alpine des Cordillères de l'Amerique du Sud. P. Bertrand: Paris, 1855.

AdDENDA.

Botanical works referred to other than Venezuelan.

Fawcett, IV.

'89. Plants collected in the Cayman Islands. Bull. bot. dept. Jamaica, 1889, no. 11, p. 6-7, Feb.

Grisebach, A. H. R.

'64. Flora of the British WVest Indian islands. Lovell Reeve \& Co.: London, 1864.

Martius, Karl F. P.

'40-06. Flora brasiliensis. Monachii and Lipsiae, 1840-1906, 15 vols., plates.

Millspaugh, C. F.

'00. Plantae Utowanae. Publ. Field Col. mus., no. 43, bot. ser., 1900, vol. 2, no. 1, p. 1-13.5. 
312 PROCEEDINGS: BOSTON SOCIETY NATURAL HISTOPY.

Pulle, A. -

'06. An enumeration of the vascular plants known from Surinam, together with their distribution and synonymy. E. J. Brill: Leiden, 1906 , pp. 555, 17 pls., map.

Schomburgk, R.

'47-'48. Reisen in Britisch-Guiana in den Jahren 1840-44. Leipzig: J. J. Weber, 1847-48, 3 vols. illustr., map.

Urban, I.

'03. Flora portoricensis, in Symbolae Antillanae, vol. 4, p. 1-352. Fratres Borntraeger: Lipsiae, 1903.

Only through Euphorbiaceae.

Printed June, 1909. 

Jonnston.- Flora of Margarita Island.

\section{EAPLANATION OF PLATES.}

PLATE 23.

Map of Caribbean Sea and its bordering lands. 


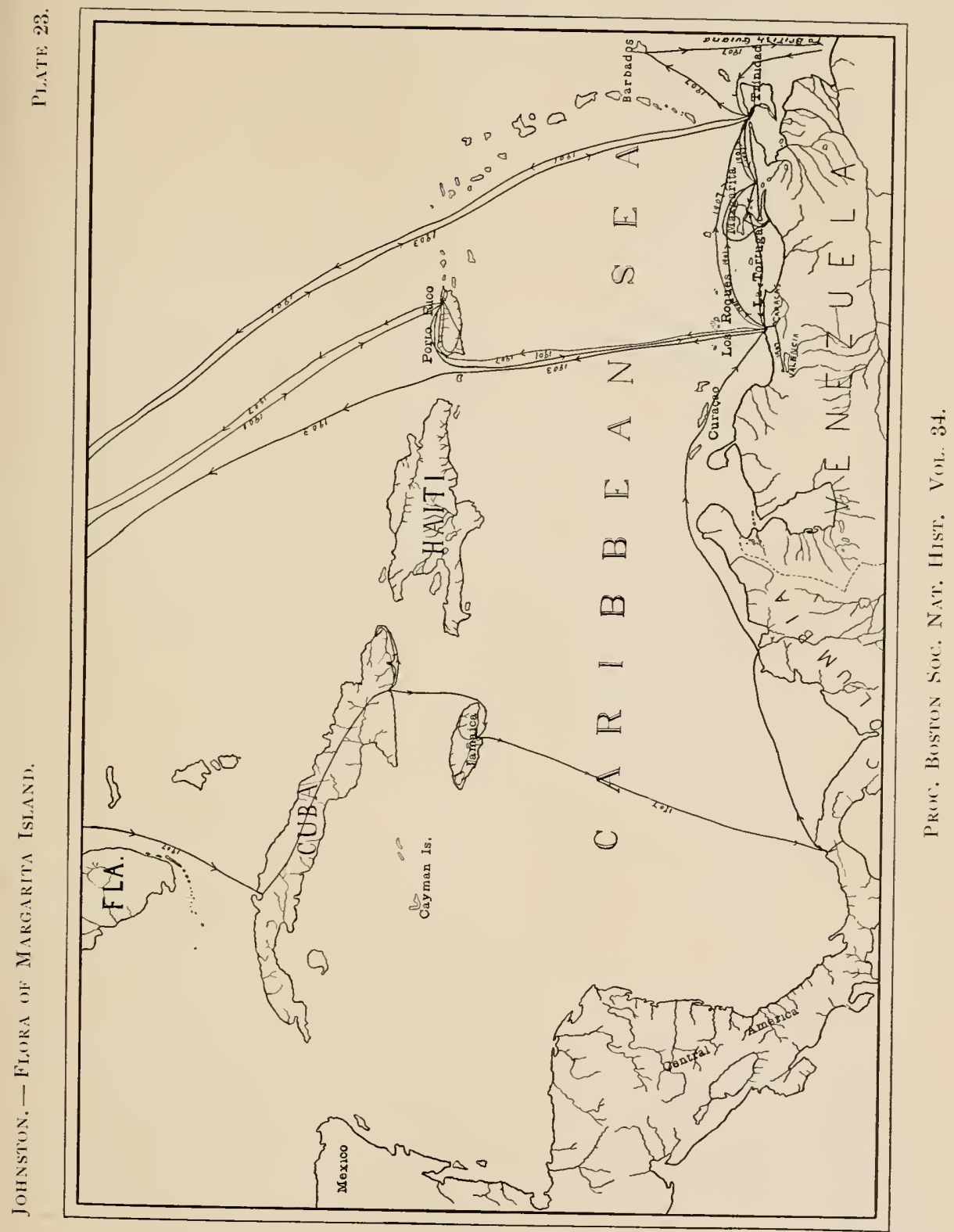




Johnston. - Flora of Margarita Island.

PLATE 24.

Map of Margarita, Coche, and Cubagua. Modified from map no. 2035 issued by the U. S. hygrographic survey. 


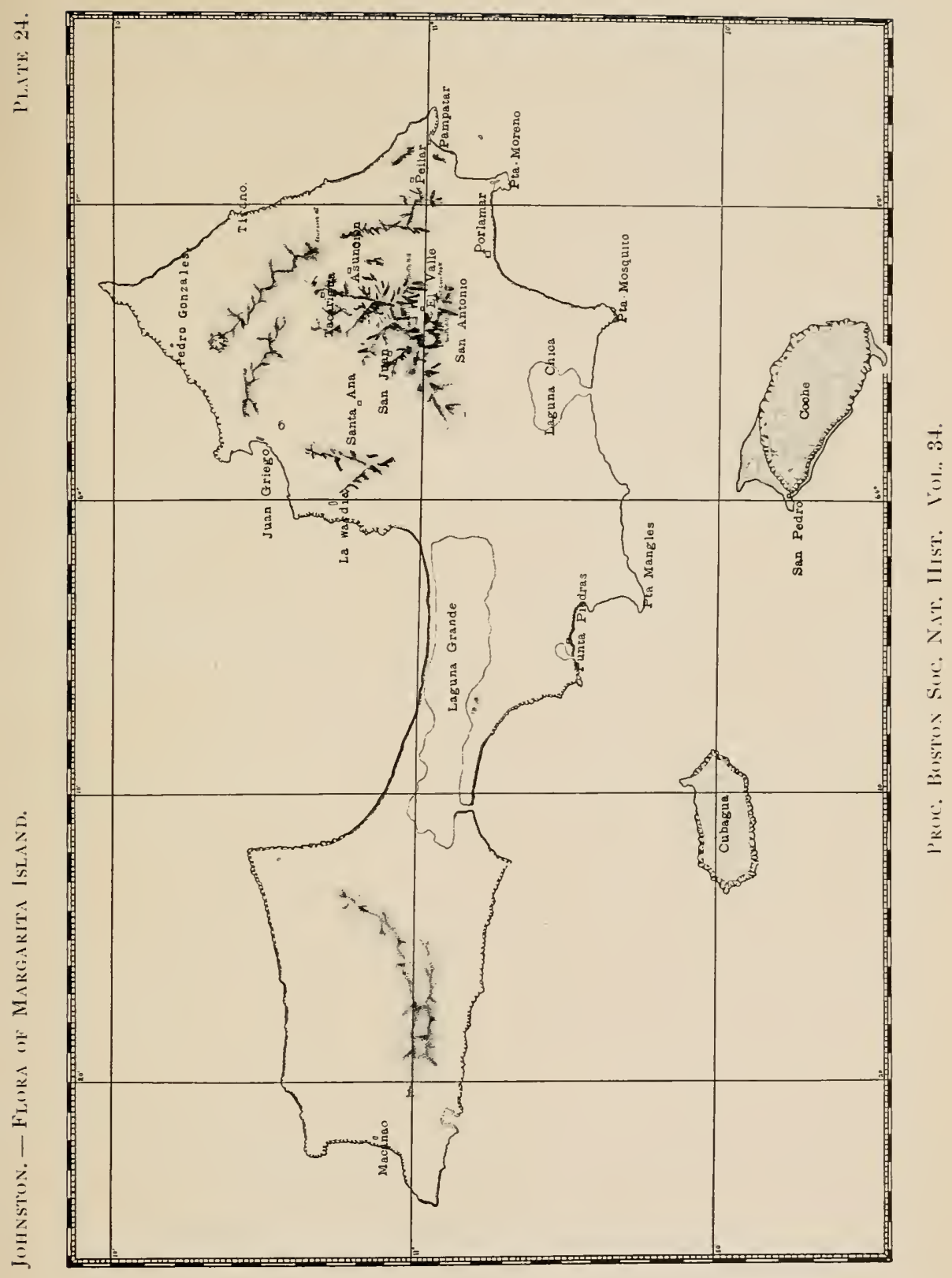




Joнхsтох. - Flora of Margarita Island.

PLATE 25.

Fig. 1. Road from Asuncion to Juan Griego. Fig. 2. Road from Porlamar to Asuncion. 

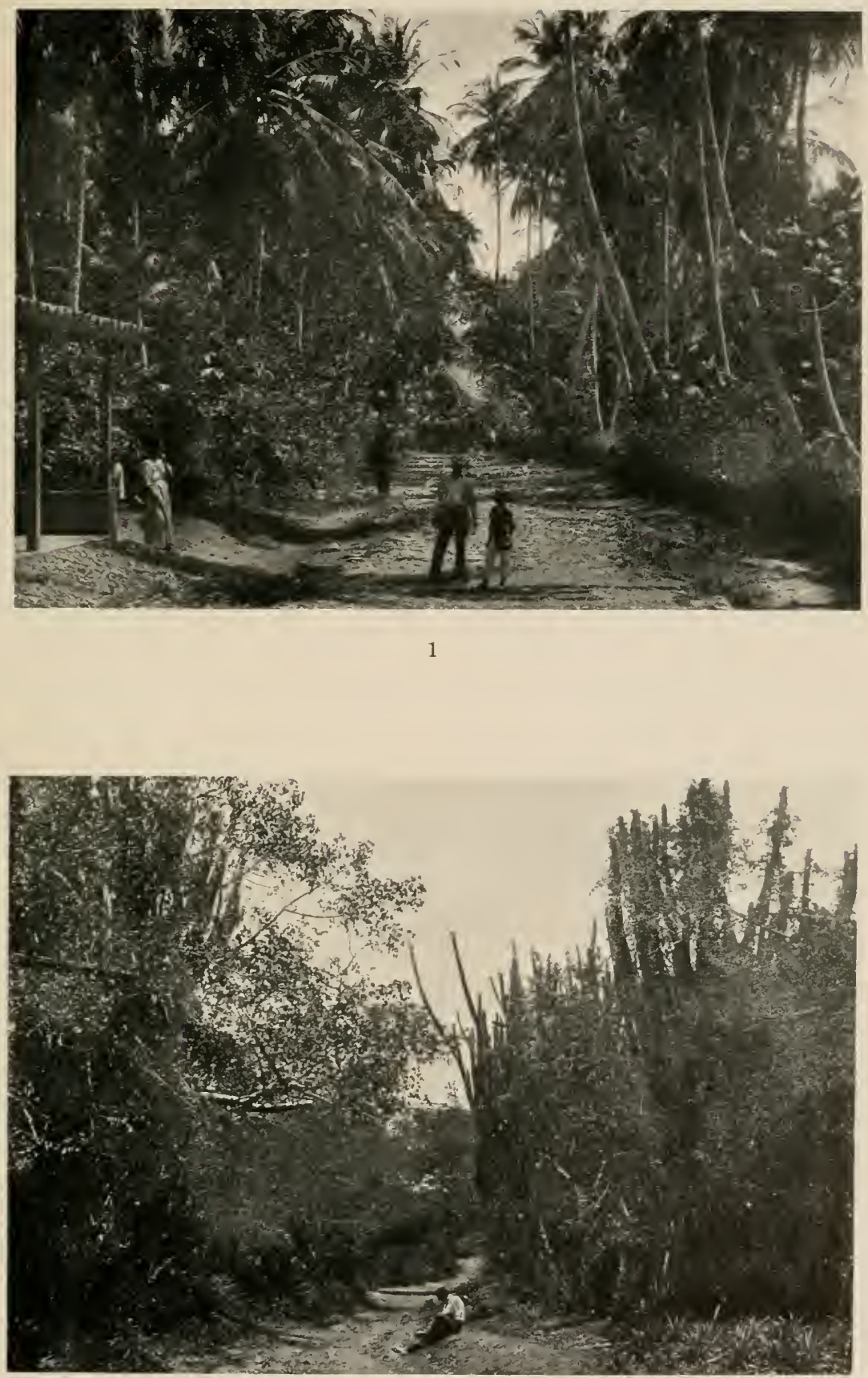


Johnston.-Flora of Margarita Isiand.

PLATE 26.

Fig. 1. Renealmia lutea.

Fig. 2. Elleanthus attenuatus. 

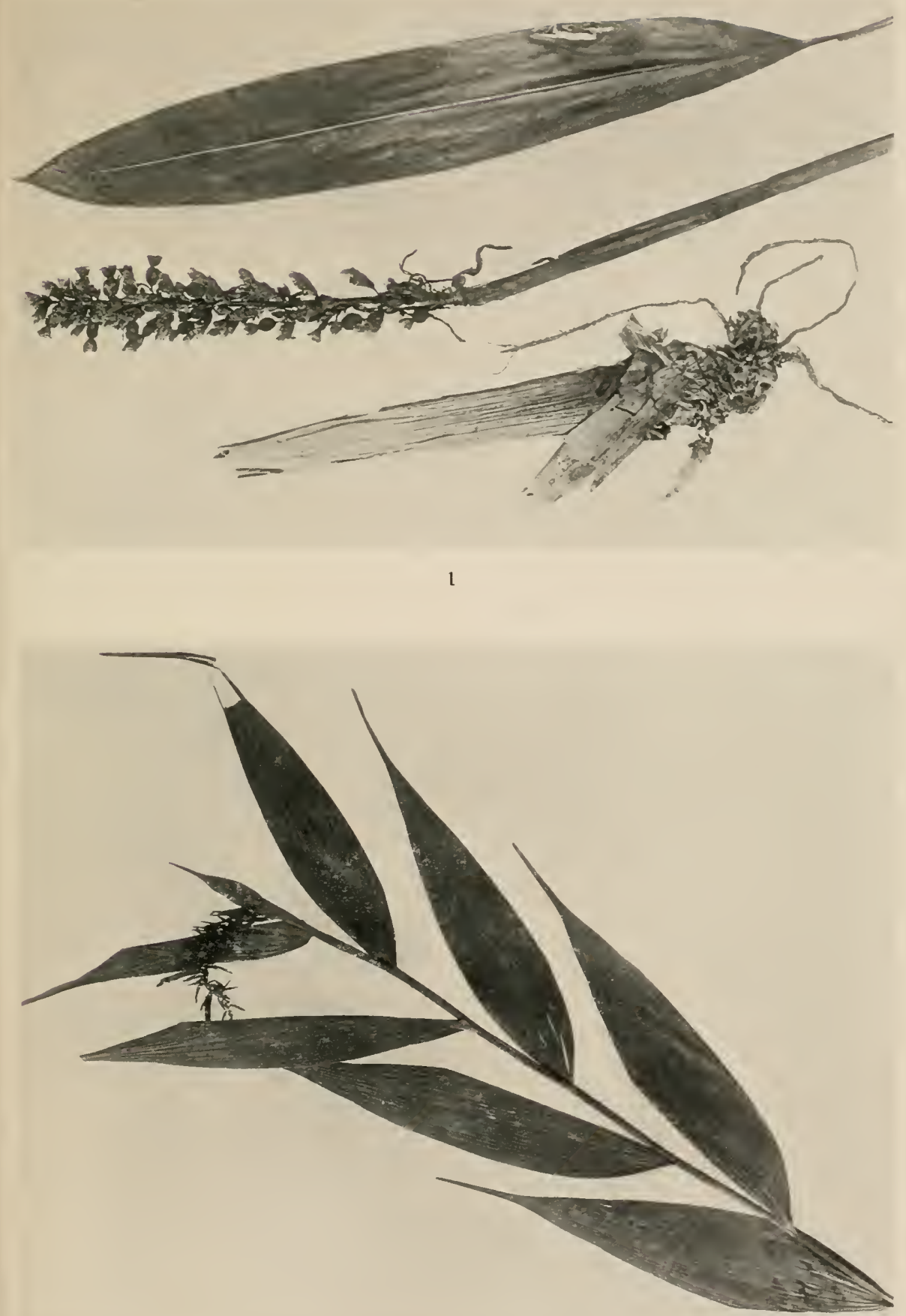


JoHNSton.- Flora of Margarita Island.

PLATE 27.

Fig. 1. Blakea monticola.

Fig. 2. Coccoloba Ernestii. 

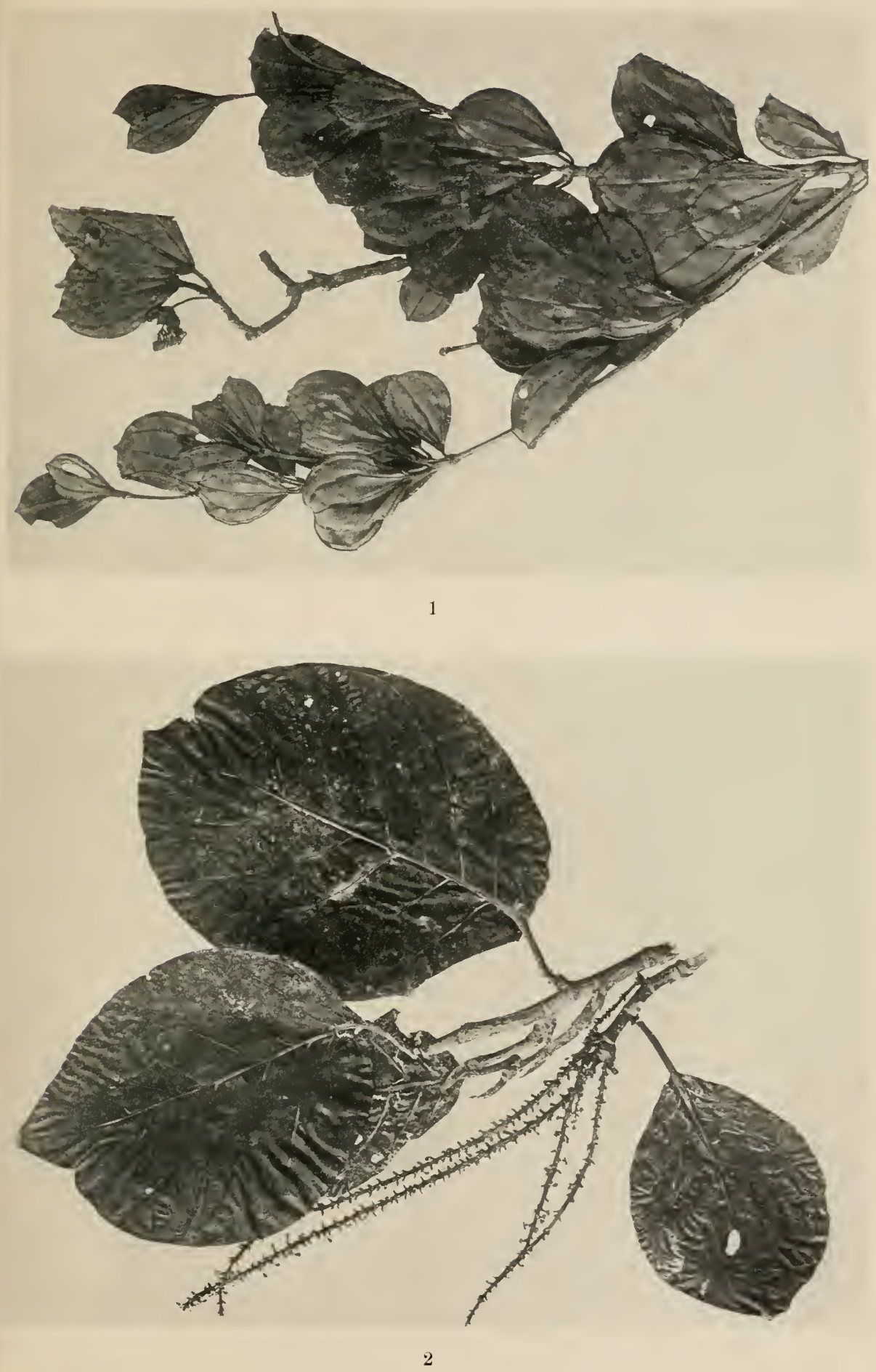

Proc. Bostux sioc, Nat. Hist. Vol. 34. 


Johnston.-Flora of Margarita Island.

PLATE 28.

Fig. 1. Inga macrantha.

Fig. 2. Machaerium striatum. 

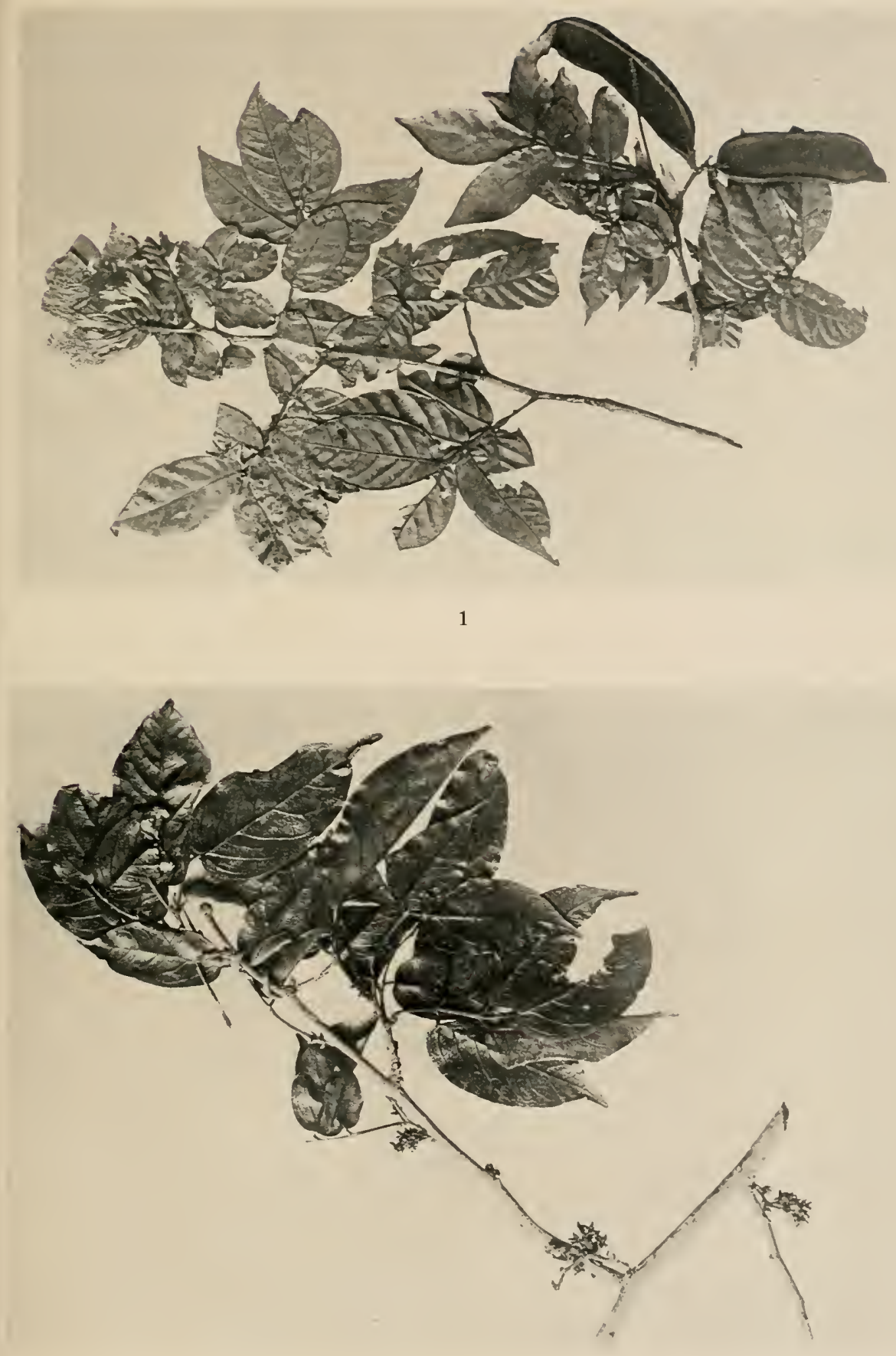


Johnston.-Flora of Margarita Island.

PLATE 29.

Fig. 1. Bignonia acuminata.

Fig. 1a. Corolla opened to show stamens.

Fig. 1b. Calyx and style.

Fig. 2. Solanum margaritense, a single flower.

Fig. 3. Chiococca micrantha, inflorescence with buds.

Fig. 3a. Flower minus corolla.

Fig. 3b. Mature ovary and old calyx.

Fig. 3c. Interpetiolar stipule. 

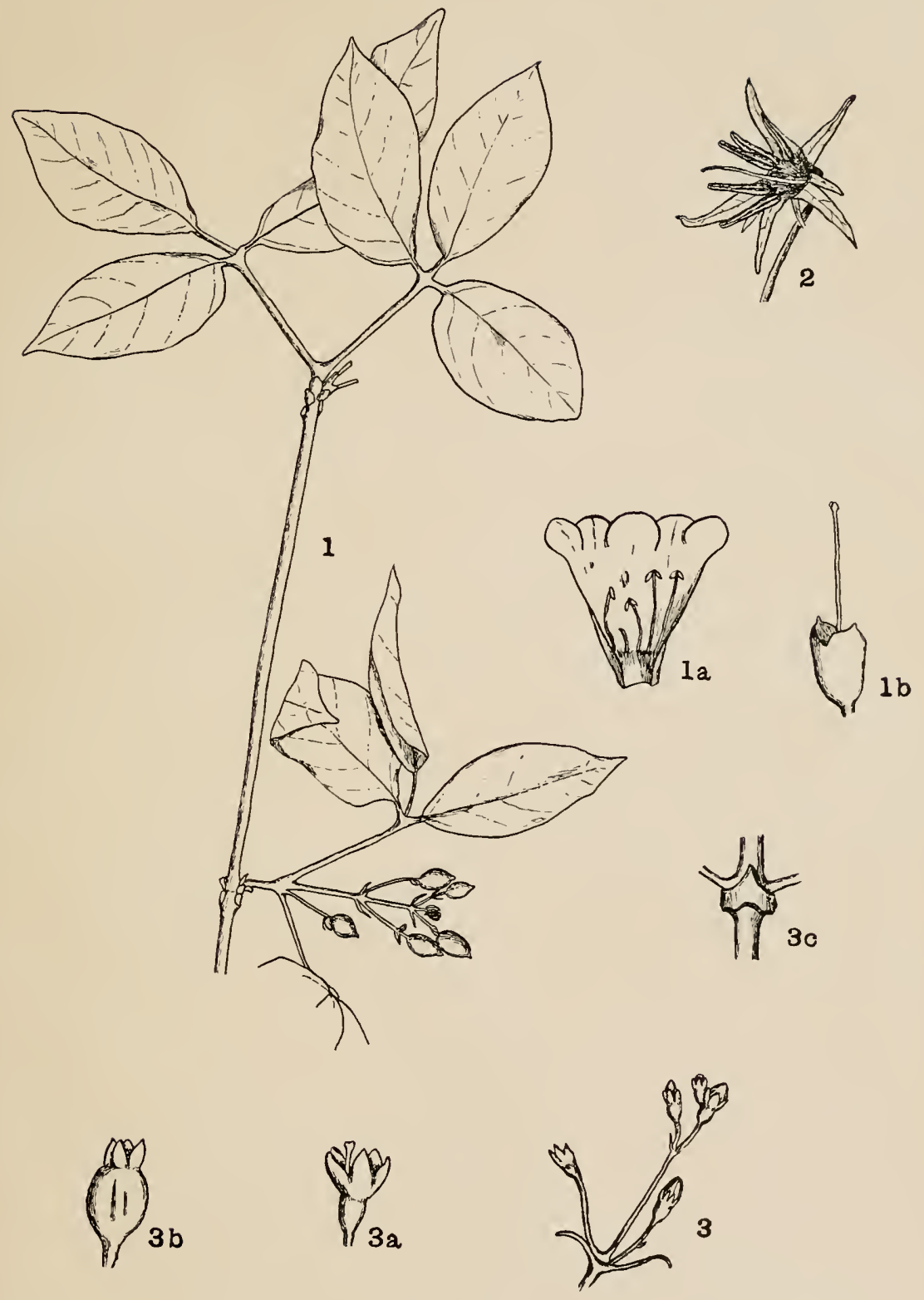

Proc. Boston Soc. Nat. Hist. Vol. 34. 


Johnston.-Flora of Margarita Island.

PLATE 30.

Fig. 1. Gliricidia lutea, standard of corolla.

Fig. $1 \mathrm{a}$ and $1 \mathrm{~b}$. Wings of corolla.

Fig. 1c. Calyx and stamens.

Fig. 1d. Keel of corolla.

Fig. 2. Croton Milleri, pistillate flower.

Fig. 2a. Ovary and style.

Fig. 2b. Petal of staminate flower.

Fig. 2c. Staminate flower.

Fig. 2d. Stamens.

Fig. 3. Croton margaritensis, staminate flower.

Fig. 3a. Pistillate flower.

Fig. 4. Argithamnia erubescens, corolla of pistillate flower.

Fig. 4a. Petal of pistillate flower.

Fig. 4b. Pistillate flower.

Fig. 4c. Ovary.

Fig. 5. Corolla of staminate flower.

Fig. 5a. Staminate flower.

Fig. 5b. Petal of staminate flower.

Fig. 5c. Stamens. 

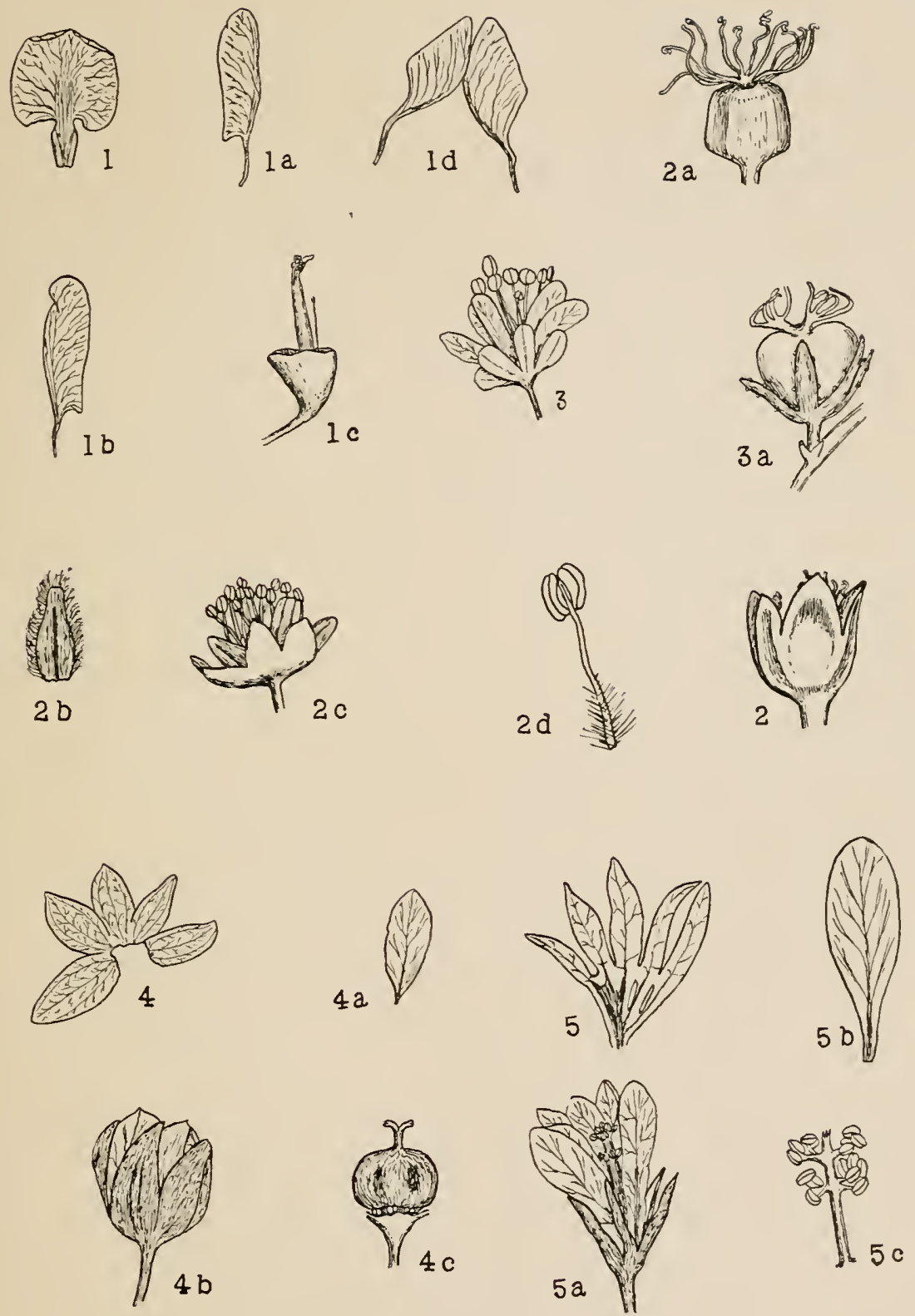

Proc. Boston Soc. Nat. Hist. Tol. 34. 


No. 6. Monograph of the Acrasieae. By E. W. Olive. 63 pp., 4 pls. 50 cts.

Vol. VI, No. 1. The anatomy and phylogeny of the Coniferales. Part 2. The Abietineae. By E. C. Jeffrey. 37 pp.. 7 pls. $\$ 1.50$.

Vol. V, No. 11. Fossil footprints of the Jura-Trias of North America. By R. S. Lull, Ph. D. 97 pp., 1 pl. $\$ 2.00$

No. 10. The anatomy and phylogeny of the Coniferales. Part 1 . The genus Sequoia. By E. C. Jeffrey. 19 pp., 4 pls. $\$ 1.00$.

No. 9. The skeletal system of Necturus maculatus Rafinesque. By H. H. Wilder, Ph.D. 53 pp., 6 pls. $\$ 1.00$.

No. 8. Observations on living Brachiopoda. By Edward S. Morse. 73 pp., 23 pls. $\$ 2.00$.

No. 7. Description of the human spines showing numerical variation, in the Warren museum of the Harvard inedical school. By Thomas Dwight. 76 pp. $\$ 1.50$.

No. 6. The anatomy and development of Cassiopea xamachana. By Robert Payne Bigelow. 46 pp., 8 pls. \$1.40.

No. 5. The development, structure, and affinities of the genus Equisetum. By E. C. Jeffrey. 36 pp., 5 pls. $\$ 1.00$.

No. 4. Localized stages in development in plants and animals. By Robert T. Jackson. 65 pp., 10 pls. $\$ 2.00$.

No. 3. Synapta vivipara: A contribution to the morphology of Echinoderms. By Hubert Lyman Clark. 35 pp., 5 pls. . $\$ 2.00$.

No. 2. Notes on the dissection and brain of the Chimpanzee "Gumbo." By Thomas Dwight. 21 pp., 4 pls. $\$ 1.00$.

No. 1. On the reserve cellulose of the seeds of Liliaceae and of some related orders. By Grace E. Cooley. 29 pp., 6 pls. $\$ 1.00$.

Vol. IV, No. 14. A bibliography of vertebrate embryology. By Charles Sedgwick Minot. $128 \mathrm{pp}$. $\$ 2.50$.

No. 13. Fusion of hands. By Thomas Dwight. 14 pp., 2 pls. 75 cts.

No. 12. The insects of the Triassic beds at Fairplay, Colorado. By Samucl H. Scudder. 16 pp., 2 pls.

No. 11. Illustrations of the Carboniferous Arachnida of North America, of the orders Anthracomarti and Pedipalpi. By Samuel H. Scudder. 14 pp., 2 pls.

No. 10. New Carboniferous Myriapoda from Illinois. By Samuel $H$. Scudder. 26 pp., 6 pls.

No. 9. New types of cockroaches from the Curboniferous deposits of the United States. By Samuel H. Scudder. 16 pp., 2 pls. Nos. 9-12, $\$ 3.25$.

No. 8. Phylogeny of the Pelecypoda; the Aviculidae and their allies. By Robert Tracy Jackson. 124 pp., 8 pls. $\$ 3.00$.

No. 7. The flora of the Kurile Islands. By K. Miyabe. 74 pp., 1 pl. $\$ 1.75$.

No. 6. The Entomophthoreae of the United States. By Roland Thaxter. 70 pp., 8 pls. $\$ 3.50$.

No. 5. The Taconic of Georgia and the report on the geology of Vermont By Jules Marcou. $28 \mathrm{pp}$., $1 \mathrm{pl}$. $\$ 1.00$.

No. 4. A study of North American Geraniaceae. By William Trelease. 34 pp., 4 pls. $\$ 1.25$.

No. 3. The introduction and spread of Pieris rapae in North America, 1S601885. By Samuel H. Scudder. 18 pp., 1 pl. 50 cts.

No. 2. The development of the ostrich fern, Onoclea struthiopteris. By Douglas H. Campbell. 36 pp., 4 pls. $\$ 1.50$.

No. 1. The significance of bone structure. By Thomas Dright. 17 pp., 3 pls. $\$ 1.25$. 


\section{Boston Society of Natural History. RECENT PUBLICATIONS.}

Proceedings. 8vo. (For price list of Memoirs, see third page of cover.)

Vol. 34, No. 5. New and little known Tipulidae. By C. IT. Johnson. 19 pp., 2 pls. 25 cts.

No. 4. Pyramidellidae of New England and the adjacent region. By P. Bartsch. 47 pp., 4 pls. 50 cts.

No. 3. Camarophorella, a Mississippian Meristelloid brachiopod. By J. E. Hyde. 31 pp., 5 pls. 50 cts.

No. 2. Foraminifera of the Woods Hole region. By J. A. Cushman. 14 pp., 1 pl. 20 cts.

No. 1. A new pteropod from New England. By C. H. Danforth. 19 pp., 4 pls. 25 cts.

Vol. 33, No. 9. Habits and structure of Scutigerella immaculata (Newport). By S. R. Williams. 25 pp., 3 pls. 35 cts.

No. 8. On Turritopsis nutricula (McCrady). By IV. K. Brooks and S. Rittenhouse. 32 pp., 6 pls. 50 cts.

No. 7. Birds of Labrador. By C. W. Townsend, M. D., and G. M. Allen, $152 \mathrm{pp} ., 1 \mathrm{pl}$. $\$ 1.00$.

No. 6. Paleontological types in the collections of the Boston society of natural history. By J. A. Cushman. $27 \mathrm{pp} .15 \mathrm{cts}$.

N8. 5. Drainage modifications in the Tallulah district. By D. IV. Johnson. 38 pp., 2 pls. 50 ets.

No. 4. Culture studies on polymorphism of Hymenomyretes. By G. R Lyman. 85 pp., 9 pls. $\$ 1.25$.

No. 3. Spermatogenesis of the Butterfly, Papilio rutulus: By J. P. Munson 82 pp., 6 pls. $\$ 1.25$.

No. 2. Terraces of the West River, Brattleboro, Vt. By E. F. Fisher. 34 pp., 11 pls. 75 cts.

No. 1. Proceedings of the Annual Meeting, May 2, 1506. $8 \mathrm{pp} .10 \mathrm{cts}$.

Vol. 32, No. 12. The annulus ventralis. By E. A. Andrews. 53 pp., 6 pls. 75 cts.

No. 11. Squamosal bone in tetrapodous Vertebrata. By F. IV. Thyng. 39 pp., 4 pls. 50 cts.

No. 10. Marine Ostracoda of Vineyard Sound and adjacent waters. By J. A. Cushman. 27 pp., 12 pls. 50 cts.

No. 9. Circulatory and respiratory systems of Desmognathus fusca. By A. B. Seelye. 23 pp., 4 pls. 35 cts.

No. 8. Anatomy of Boophilus annulatus Say. By S. R. Williams. 22 pp., 5 pls. 50 cts.

No. 7. Birds of the southern Lesser Antilles. By A. H. Clark. 110 pp. $\$ 1.00$.

No. 6. Alimentary tract of the mosquito. By M. T. Thompson. 58 .pp., 6 pls. 75 cts.

No. 5. Proceedings of the Annual Meeting, May 3, 1905.29 pp. 20 cts.

No. 4. Development of ascus and spore formation in Ascomycetes. By J. H. Faull. 37 pp., 5 pls. 50 cts.

No. 3. General anatomy of Typhlomolgerathbuni. By E. T. Emerson, 2d., 33 pp., 5 pls. 50 cts.

No. 2. List of shell-bearing Mollusca of Frenchman's Bay, Maine. By D. Blaney. 19 pp., 1 pl. 25 cts.

No. 1. Proceedings of the Annual Meeting, May 4, 1904. 21 pp. 20 cts.

Vol. 31, No. 10. The anatomy and development of the terrestrial nemertean (Geonemertes agricola) of Bermuda. By W. R. Coe. 40 pp., 3 pls. 50 cts.

No. 9. North American Ustilagineae. By G. P. Clinton. 200 pp. $\$ 2.00$.

No. 8. Pyenogonida collected at Bermuda in the summer of 1903 . By L. J. Cole. 14 pp., 3 pls. 25 cts.

No. 7. Trichomes of the root in vascular Cryptogams and Angiosperms. By R. G. Leavitt. 41 pp., 4 pls. 50 cts.

No. 6 . Contributions from the Gray herbarium of Harvard university. New series.- No. 27. By B. L. Robinson. 25 pp. 25 cts.

No. 5. Observations on the cytology of Araiospora pulchra Thaxter. By C. A. King. 35 pp., 5 pls. 50 cts.

No. 4. The metamorphoses of the hermit crab. By M. T. Thompson. 63 pp., 7 pls. 75 cts.

No. 3. Systematic results of the study of North American land mamnals during the years 1901 and 1902. By G. S. Miller, Jr, and J. A. G. Rehn. 85 pp. 50 cts. 


QK 250.J6 York Botanical Garden Library

Johnston, John Robe/Flora of the islands

,

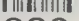

3518500101 6839 
$\frac{5 i}{2}$ 